Draft version SePtember 2, 2021

Preprint typeset using $\mathrm{LATE}_{\mathrm{E}} \mathrm{X}$ style emulateapj v. 12/16/11

\title{
STATISTICS OF "COLD” EARLY IMPULSIVE SOLAR FLARES IN X-RAY AND MICROWAVE DOMAINS
}

\author{
Alexandra L. Lysenko \\ Ioffe Institute, Polytekhnicheskaya, 26, St. Petersburg, 194021 - Russia \\ Alexander T. Altyntsev \\ Institute of Solar-Terrestrial Physics (ISZF), Lermontov st., 126a, Irkutsk, 664033 - Russia \\ Natalia S. Meshalkina \\ Institute of Solar-Terrestrial Physics (ISZF), Lermontov st., 126a, Irkutsk, 664033 - Russia \\ DMITRIY ZHDANOV \\ Institute of Solar-Terrestrial Physics (ISZF), Lermontov st., 126a, Irkutsk, 664033 - Russia \\ Gregory D. Fleishman \\ New Jersey Institute of Technology, University Heights, Newark, NJ 07102-1982 - United States and \\ Ioffe Institute, Polytekhnicheskaya, 26, St. Petersburg, 194021 - Russia \\ (Received 2017/10/26; Revised 2017/11/17; Accepted 2018/02/22) \\ Draft version September 2, 2021
}

\begin{abstract}
Solar flares often happen after a preflare / preheating phase, which is almost or entirely thermal. In contrast, there are the so-called early impulsive flares that do not show a (significant) preflare heating but instead often show the Neupert effect-a relationship where the impulsive phase is followed by a gradual, cumulative-like, thermal response. This has been interpreted as a dominance of nonthermal energy release at the impulsive phase, even though a similar phenomenology is expected if the thermal and nonthermal energies are released in comparable amounts at the impulsive phase. Nevertheless, some flares do show a good quantitative correspondence between the nonthermal electron energy input and plasma heating; in such cases the thermal response was weak, which results in calling them "cold" flares. We undertook a systematic search of such events among early impulsive flares registered by Konus- Wind instrument in the triggered mode from 11/1994 to 04/2017 and selected 27 cold flares based on relationships between HXR (Konus-Wind) and SXR (GOES) emission. For these events we put together all available microwave data from different instruments. We obtained temporal and spectral parameters of HXR and microwave emissions of the events and examined correlations between them. We found that, compared with a 'mean' flare, the cold flares: (i) are weaker, shorter, and harder in the X-ray domain; (ii) are harder and shorter, but not weaker in the microwaves; (iii) have a significantly higher spectral peak frequencies in the microwaves. We discuss the possible physical reasons for these distinctions and implication of the finding.
\end{abstract}

Subject headings: Sun: flares - Sun: radio radiation - Sun: X-rays

\section{INTRODUCTION}

Explosive energy release that results in efficient acceleration of charged particles and heating of the ambient plasma is ubiquitous in the astrophysics. In the solar atmosphere this energy release is observed as transient brightening at various wavelengths, most notably during solar flares. In the solar flares the excessive magnetic energy can be promptly released at a time scale as short as seconds, while generating nonthermal particles with high energies as large as $1 \mathrm{MeV}$ and heating the ambient plasma up to several dozens million Kelvin. Although particle acceleration and plasma heating are typical for many astrophysical objects, the solar flares offer a unique natural laboratory, where these processes can be studied at extremely short spatial and temporal scales needed to address the key dynamics of the explosive energy release.

alexandra.lysenko@mail.ioffe.ru
It is puzzling that the proportions of the energy that initially go to either particle acceleration or plasma heating vary dramatically between different solar flares. Indeed, there are entirely thermal flares (Garv \& Hurford 1989; Battaglia et al. 2009; Fleishman et al. 2015), where no nonthermal emission is detected, while in some flares an exceptionally strong nonthermal emission is accompanied by a very modest thermal component (White et al. 1992; Bastian et al. 2007; Fleishman et al. 2011; Masuda et al. 2013). Not surprisingly, there are cases with all possible proportions between these mentioned extremes of purely thermal and entirely nonthermal flares.

It is not yet clear what physical process or parameter combination is decisive for the initial energy partitions in the flares, neither what is the fundamental difference between a 'normal' solar flare, in which the thermal and nonthermal energies are initially comparable, and those dominated by the nonthermal component. In this study 
we are going to advance these questions using a statistical comparison between events from various subgroups described below. There are numerous statistical studies of solar flares in the X-ray and microwave domains that employ data from many past or existing instruments. In particular, Tanaka et al. (1983), based on HINOTORI data, and Dennis (1985), based on SMM/HXRBS data, performed the statistical analysis of the X-Ray bursts registered during solar cycle 21 and revealed three types of X-Ray solar flares: the first type represents soft hot flares with minor HXR emission, the flares of the second type have noticeable HXR emission, impulsive time profiles and soft-hard-soft spectral evolution with typical power-law spectral indices between $\sim 2$ and $\sim 8$, and the flares of the third type are characterized by extended durations, soft-hard-harder spectral evolution and powerlaw indices $\leq 4.5$ (Bai \& Sturrock 1989). Dennis (1985) also established that the occurrence rate distribution of the HXR peak fluxes obeys a power-law with index 1.8. Later, power-laws were also found for distributions of HXR fluences with power-law indices between -1.53 and -1.77 and HXR durations with power-law indices between -1.76 and -2.54 (Crosby et al. 1993; Aschwanden 2011). The statistical comparison between HXR and microwave flare components performed by Kosugi et al. (1988) shows, that HXR emission in impulsive flares at $\sim 100 \mathrm{keV}$ and microwave emission at $17 \mathrm{GHz}$ are highly correlated and presumably are the results of nonrelativistic down-streaming electrons, while in extended flares microwaves are emitted by relativistic electrons trapped in coronal loops. Silva et al. (2000) performed the analysis of correlations between paramaters of HXR burts registered by CGRO/BATSE and microwave by OVSA, this analysis also revealed that HXR fluxes in $\leq 200 \mathrm{keV}$ and microwave fluxes are generally correlated, while HXR and microwave spectral parameters are often unrelated. One of the most detailed statistical studies in the microwave domain was performed by Nita et al. (2004) for flares registered by the Owens Valley Solar Array (OVSA) during 2001-2002.

Su et al. (2008), based on the RHESSI data, introduced three solar flare types according to their relationship between thermal and nonthermal emission. Type 1, "accordantly gradual flares", are purely thermal with no obvious emission above $25 \mathrm{keV}$. Type 2 flares, called "accordantly impulsive flares", demonstrate impulsive nonthermal emission in HXRs along with more gradual thermal SXR emission. In the case of type 3 flares, "early impulsive flares", first proposed by Sui et al. (2007), impulsive nonthermal phase is followed by thermal emission. In a subset of impulsive flares, a so-called Neupert effect is observed (Neupert 1968). Originally, Neupert (1968) discovered a correlation between the flare time profiles in SXR, where the thermal emission is observed, and the cumulative integral of the time profile in microwaves, where the emission is generated by nonthermal particles. Later, a similar relationship was revealed between SXRs and HXRs. Taken at the face value, the Neupert effect implies that the thermal plasma is heated by nonthermal electrons. However, this heating by the nonthermal electrons might not be the only heating occurring during the flare. Indeed, if the thermal plasma is somehow impulsively heated directly by the magnetic energy release at the same time scale as that of the electron acceleration, the light curves of thermal emission will still demonstrate the Neupert effect because the thermal plasma cooling is a much slower process than the impulsive energy release. Such additional heating was proposed by Veronig et al. (2005) in a subset of events that demonstrate the phenomenology of the Neupert effect. In additions, other ways of plasma heating are observed or implied in solar flares, so the Neupert effect is not always observed (Su et al. 2008; Battaglia et al. 2009).

Although in a general case the presence of the Neupert effect does not guarantee that the plasma heating is solely driven by the nonthermal electrons, it would not be unreasonable to expect that there are flares in which this heating mechanism does dominate. Most likely, such flare would represent a subset of early impulsive flares, whose thermal emission is very low before the impulsive phase, so the thermal plasma is likely heated by almost only nonthermal particles. Such cases, called "cold" solar flares (CSFs hereafter), that show very low thermal response relative to nonthermal energy of accelerated particles, have been reported in previous case studies (White et al. 1992; Bastian et al. 2007; Fleishman et al. 2011; Masuda et al. 2013). However, the criteria to classify a given flare as a "cold" one were somewhat subjective; typically, the reported CSFs showed a noticeable HXR and/or microwave burst that happened without any reported GOES flare. Yet, no formal criterion has been developed of how weak the thermal response must be compared to the nonthermal emission for the flare to be classified as a cold one. Accordingly, with all the variety of statistical studies described above, no focused statistical study on the flares with weak thermal response, the "cold" flares, has yet been available.

It is important to realize that there are two formal reasons to render the thermal SXR emission low: (a) low plasma temperature (as in the cases reported by Bastian et al. 2007; Masuda et al. 2013) or (b) low emission measure due to either a low plasma density (as in the tenuous flare reported by Fleishman et al. 2011) or a small volume of the flaring loop (as in the main flaring loop in the flare reported by Fleishman et al. 2016)]; thus, in case (b) the observed thermal response can remain low even if the plasma temperature is high. With this reservation, here we will use the term "cold flare" for any event with a reasonably weak thermal signature (see below for formal criteria), because it is not at all easy to sort out cases (a) and (b) at the stage of the event selection.

Here we take advantage of availability of an almost uniform database of solar flares recorded by the KonusWind Aptekar et al. 1995) during two solar cycles (between November, 1994 and April, 2017) to build a statistically significant subset of the cold flares and study their properties as compared to other flares. Based on the performed statistical analysis, we discuss what flare properties or parameter combination make the CSF different from the 'normal' flare and what are the likely main causes of the apparent lack of thermal emission in

\footnotetext{
${ }^{1}$ In this flare the thermal response was very weak at the impulsive phase because a small loop with a correspondingly small volume produced the impulsive HXR and microwave emission. However, one more, much bigger loop was also involved in the flaring, which was responsible for a delayed thermal response in this, rather unusual event.
} 
this class of solar flares.

\section{INSTRUMENTATION AND EVENT SELECTION}

\subsection{X-ray Domain: Uniform Input from the KONUS-WIND and the GOES}

Given that CSFs occur relatively seldom, the statistical study of these events requires a reasonably long series of observations. From this perspective we employ hard Xray (HXR) data from the Konus-Wind and soft X-ray (SXR) data from the GOES; both data sets are available over the time period longer than two full solar cycles (White et al. 2005).

\subsubsection{GOES Soft X-ray Data}

Spacecrafts of GOES (Geostationary Operational Environmental Satellite) series observe the Sun almost continuously since 1974. We used data of GOES X-ray sensors in two broadband SXR channels, softer channel 1$8 \AA$ and harder channel $0.5-4 \AA$, with temporal resolution varied from $3 \mathrm{~s}$ to $2.046 \mathrm{~s}$ during observational history.

\subsubsection{KONUS-WIND Hard X-ray Solar Data}

Konus-Wind was launched on 1 November 1994 on board of the Wind spacecraft to detect gamma-ray bursts and solar flares in HXR domain. It operates in interplanetary space and since July 2004 is located near Lagrange point L1 at $\sim 5$ light seconds from the Earth. KonusWind consists of two identical $13 \mathrm{~cm} \mathrm{x} 7.5 \mathrm{~cm} \mathrm{NaI(Tl)}$ detectors S1 and S2 with Be entrance window. Detectors are located on the opposite sides of the spacecraft observing the southern and the northern ecliptical hemispheres correspondingly (Aptekar et al. 1995).

The Konus- Wind works in two modes - the waiting mode and the triggered mode. In the waiting mode, only the light curves with accumulation time $2.944 \mathrm{~s}$ are available in three wide energy bands: G1 (nominal range 13$50 \mathrm{keV}$ ), G2 (nominal range 50-200 keV), G3 (nominal range $200-750 \mathrm{keV}$ ). The Konus- Wind energy boundaries changed during its observational history within a factor of 2.0 for detector S1 and 1.5 for detector S2.

Switching to the triggered mode occurs at a statistically significant background excess on $1 \mathrm{~s}$ or $140 \mathrm{~ms}$ timescale in energy band G2. In the triggered mode, the light curves are recorded in the same three energy bands with the high time resolution (varying from 2 to $256 \mathrm{~ms}$ as the burst progresses) during $230 \mathrm{~s}$ along with accumulation of 64 multichannel energy spectra. Multichannel spectra are measured in partially overlapping energy ranges: nominal boundaries are $13-750 \mathrm{keV}$ for the first range and $250 \mathrm{keV}-15 \mathrm{MeV}$ for the second range. Now the spectral ranges have changed to $\sim 25 \mathrm{keV}-18 \mathrm{MeV}$ for the $\mathrm{S} 1$ detector and $\sim 20 \mathrm{keV}-15 \mathrm{MeV}$ for the S2 detector from the original $13 \mathrm{keV}-10 \mathrm{MeV}$. Each energy range consists of 63 energy channels. Accumulation time for each of the first 4 spectra is $64 \mathrm{~ms}$, while the accumulation time varies for the subsequent 52 spectra from $256 \mathrm{~ms}$ to $8.192 \mathrm{~s}$ according to the count rate in G2 energy band: for stronger HXR flux the accumulation time is proportionally shorter. For the latest eight spectra

2 Light curves and spectra of solar flares registered by Konus-Wind in the triggered mode can be found at http://www.ioffe.ru/LEA/kwsun/ the accumulation time is fixed at $8.192 \mathrm{~s}$. When accumulation of the triggered mode light curves and energy spectra has been completed, both triggered and waiting mode measurements are interrupted by a gap of $\sim 1$ hour because of the data readout.

Due to adopted implementation of the trigger algorithm, in the triggered mode the Konus-Wind registers only reasonably hard flares showing a rather rapid increase in HXR intensity, while for softer and smoother events only waiting mode data are available. Because of the limited duration of the trigger record $(\sim 240 \mathrm{~s}$ for time history and $\sim 480$ s maximum for energy spectra) for longer flares the recording ends before the end of the flare (Pal'shin et al. 2014).

Given the lack of spatial resolution, an algorithm is needed to distinguish solar bursts from other astrophysical sources. There is a number of criteria to conclude if a Konus-Wind triggered mode event is a solar flare or not. First, as Konus-Wind detectors are pointed transversely to ecliptic plane, emission from solar flares reaches Konus- Wind at angles $\sim 90^{\circ}$ and is seen in both detectors $\mathrm{S} 1$ and $\mathrm{S} 2$ - in one detector in the triggered mode, while in the other-in the waiting mode (the trigger may only occur in one detector at a time). Second, we check the GOES X-ray event list 3 for event notification or look for increase in the GOES SXR flux at that time. Also the Fermi trigger reports may be used for a subset of jointly observed events.

\subsubsection{Selection of Early Impulsive Flares}

To form an initial list of the event candidates, we employed the following formal criterion for the "solar flare"like burst registered by Konus-Wind in the triggered mode to be listed as early impulsive flare: we require that no GOES X-ray event has been reported at the time of the Konus-Wind trigger. This means that either the corresponding GOES event began later than the KonusWind trigger time or there was no reported GOES event at all. The goal of using that strict criterion is to exclude events with SXR emission due to plasma preheating by any other agents than the nonthermal flare-accelerated electrons.

This automatic search yielded 84 events. Some of these events were then discarded manually because of failures in the Konus-Wind data, some events were identified as false alarms caused by energetic particles, not HXR emission. Three events are missing from the GOES event list for an unknown reason, though they demonstrate noticeable increase of GOES 1-8 $\AA$ flux before the HXR impulsive phase, and, thus, do not obey the entry criterion. Two event were discarded due to failures in the GOES data.

Finally, we approved 42 solar flares, whose properties are consistent with those of early impulsive flares proposed by Sui et al. (2007): no increase in SXR flux must be seen earlier than $30 \mathrm{~s}$ prior to the increase in the HXR flux. For all forty two events there is no corresponding reported GOES flare. Thus, our criterion for the early impulsive flare selection is really rather strict. The absence of a solar flare in the GOES event implies that

\footnotetext{
${ }^{3}$ GOES event list/ftp://ftp.swpc.noaa.gov/pub/indices/events/

${ }^{4}$ Fermi trigger information/https://gcn.gsfc.nasa.gov/fermi_grbs.html/
} 
TABLE 1

KONUS- Wind COLD SOLAR FLARE LiST.

\begin{tabular}{|c|c|c|c|c|c|}
\hline $\mathrm{N}$ & $\operatorname{strID}^{*}$ & Date & $t_{0}{ }^{* *}$, hh:mm:ss & Coordinates & Instrument $^{* * *}$ \\
\hline 1 & SOL1998-05-07T19952 & 1998-Мay-07 & $05: 32: 37.072$ & {$\left[554^{\prime \prime}, 495^{\prime \prime}\right]$} & EIT, $195 \AA$ \\
\hline 2 & SOL1999-06-19T82485 & 1999-Jun-19 & $22: 54: 49.788$ & {$\left[-916^{\prime \prime}, 233^{\prime \prime}\right]$} & NoRH \\
\hline 3 & SOL1999-07-30T82686 & 1999-Jul-30 & $22: 58: 09.675$ & {$\left[-490^{\prime \prime}, 320^{\prime \prime}\right]$} & NoRH \\
\hline 4 & SOL1999-11-09T30381 & 1999-Nov-09 & $08: 26: 21.703$ & $\ldots$ & $\ldots$ \\
\hline 5 & SOL1999-11-14T53708 & 1999-Nov-14 & $14: 55: 08.244$ & $\ldots$ & $\ldots$ \\
\hline 6 & SOL1999-12-02T72060 & 1999-Dec-02 & 20:01:00.012 & $\ldots$ & $\ldots$ \\
\hline 7 & SOL2000-03-10T15704 & 2000-Mar-10 & $04: 21: 48.688$ & {$\left[-776^{\prime \prime},-211^{\prime \prime}\right]$} & NoRH \\
\hline 8 & SOL2000-03-18T08707 & 2000-Mar-18 & $02: 25: 10.567$ & {$\left[717^{\prime \prime},-294^{\prime \prime}\right]$} & SSRT \\
\hline 9 & SOL2000-05-18T26517 & 2000-May-18 & $07: 21: 59.706$ & {$\left[-33^{\prime \prime},-257^{\prime \prime}\right]$} & SSRT \\
\hline 10 & SOL2000-05-18T82777 & 2000-May- 18 & $22: 59: 39.777$ & {$\left[177^{\prime \prime \prime},-319^{\prime \prime}\right]$} & NoRH \\
\hline 11 & SOL2001-10-12T27630 & 2001-Oct-12 & $07: 40: 31.941$ & {$\left[-900^{\prime \prime}, 238^{\prime \prime}\right]$} & SSRT \\
\hline 12 & SOL2001-11-01T55062 & 2001-Nov-01 & $15: 17: 42.772$ & {$\left[320^{\prime \prime}, 125^{\prime \prime}\right]$} & OVSA \\
\hline 13 & SOL2002-05-29T27586 & 2002-May-29 & $07: 39: 46.864$ & {$\left[-343^{\prime \prime}, 122^{\prime \prime}\right]$} & SSRT \\
\hline 14 & SOL2002-08-10T85808 & 2002-Aug-10 & $23: 50: 09.293$ & {$\left[-938^{\prime \prime},-79^{\prime \prime}\right]$} & NoRH \\
\hline 15 & SOL2002-08-18T83478 & 2002-Aug-18 & $23: 11: 19.740$ & {$\left[300^{\prime \prime},-300^{\prime \prime}\right]$} & OVSA \\
\hline 16 & SOL2002-08-20T71727 & 2002-Aug-20 & $19: 55: 28.476$ & & \\
\hline 17 & SOL2003-10-23T80262 & 2003-Oct-23 & $22: 17: 39.620$ & {$\left[-938^{\prime \prime},-307^{\prime \prime}\right]$} & RHESSI \\
\hline 18 & SOL2005-09-08T08145 & 2005-Sep-08 & $02: 15: 49.996$ & & \\
\hline 19 & SOL2011-09-19T27816 & 2011-Sep-19 & $07: 43: 40.791$ & {$\left[-806^{\prime \prime}, 345^{\prime \prime}\right]$} & RHESSI \\
\hline 20 & SOL2012-07-08T09826 & 2012-Jul-08 & $02: 43: 50.647$ & {$\left[894^{\prime \prime},-206^{\prime \prime}\right.$} & NoRH \\
\hline 21 & SOL2013-11-05T13819 & 2013-Nov-05 & $03: 50: 24.588$ & {$\left[-771^{\prime \prime},-250^{\prime \prime}\right]$} & NoRH \\
\hline 22 & SOL2014-01-02T20697 & 2014-Jan-02 & $05: 45: 01.390$ & {$\left[-948^{\prime \prime},-83^{\prime \prime}\right]$} & NoRH \\
\hline 23 & SOL2014-01-31T60753 & 2014-Jan-31 & $16: 52: 37.461$ & {$\left[-504^{\prime \prime}, 331^{\prime \prime}\right]$} & RHESSI \\
\hline 24 & SOL2014-02-08T20965 & 2014-Feb-08 & $05: 49: 29.848$ & {$\left[856^{\prime \prime},-150^{\prime \prime}\right]$} & RHESSI \\
\hline 25 & SOL2014-10-18T10152 & 2014-Oct-18 & $02: 49: 17.710$ & {$\left[-909^{\prime \prime},-335^{\prime \prime}\right]$} & RHESSI \\
\hline 26 & SOL2014-10-27T11681 & 2014-Oct-27 & $03: 14: 46.862$ & {$\left[640^{\prime \prime},-290^{\prime \prime}\right]$} & RHESSI \\
\hline 27 & SOL2015-05-07T45695 & 2015-May-07 & $12: 41: 40.415$ & & $\ldots$ \\
\hline
\end{tabular}

${ }^{*}$ Here $\mathrm{T}^{* * * * *}$ is the Konus- Wind trigger time in seconds without time of light propagation corrections to match the format used in http://www.ioffe.ru/LEA/kwsun/

** The Konus-Wind trigger time after corrections for the light propagation to the Earth are applied.

*** The instrument used for the event localization.

it did not obey the adopted GOES flare selection criterion: a solar flare is listed as a GOES flare if it demonstrates monotonous flux increase in the GOES 1-8 $\AA$ channel during at least 1 minute as compared to previous 3 minutes. Thus, short events and events during unstable background could be missed from this list. In what follows, see Section 3.1. based on cross-correlation analysis between HXR and SXR data, we selected only 27 cold out of these 42 early impulsive flares for a more detailed analysis, listed in Table 1 Konus- Wind time profiles for these events are presented in Figures 1 and 2

\subsubsection{Selection of Reference Flares}

Because of the trigger nature of the Konus-Wind instrument, which records only reasonably hard impulsive HXR bursts (see Section 2.1.2 above), we could not make a meaningful direct comparison of our cold flare subset with previously available statistical studies. This forced us to form and use a reference set of other bursts recorded by the Konus-Wind in the triggered mode, with which the CSFs are to be compared. Accordingly, from all $1000+$ solar flares recorded by Konus- Wind in the triggered mode, for the reference set we selected flares that (i) have constant Konus-Wind and GOES background and (ii) fully covered by the Konus-Wind time history record (both in the waiting and in the triggered mode), i. e. which ended before the end of the trigger record (but may begin in the waiting mode record before the trigger mode record begin). This last condition implies discarding long-duration flares. In this way we selected $405 \mathrm{C}, \mathrm{M}$, and X GOES class flares to form the reference set.

\subsection{Microwave domain: nonuniform input}

Historically, the microwave data played a primary role in identification and analysis of the cold flares (White et al. 1992; Bastian et al. 2007; Fleishman et al. 2011; Masuda et al. 2013; Fleishman et al. 2016); thus, we have to use all available microwave data fully. Unfortunately the only quasi-uniform set of radio instruments is Radio Solar Telescope Network (RSTN, Guidice et al. 1981), which has a lot of disadvantages including clock errors, calibration errors, big gaps between the working frequencies, and a limited spectral coverage. For this reason, in addition to RSTN, we use several other radio instruments; namely the Owens Valley Solar Array (OVSA, Hurford et al. 1984; Garv \& Hurford 1994), the Nobeyama Radio Polarimeters (NoRP, Torii et al.|1979), the Solar Radio Spectropolarimeters (SRS, Muratov 2011), the Badary Broadband Microwave Spectropolarimeters (BBMS, Zhdanov \& Zandanov 2015), and the Kislovodsk Mountain Astronomical Station of the Pulkovo Observatory (KMAS, Shramko et al. 2011); see Table 2. This approach, however, has a disadvantage of making the data input nonuniform. We will discuss implications of this nonuniformity later.

\subsubsection{Radio Instruments}

RSTN provides radio data with 1 second temporal resolution taken at 8 selected frequencies $(245 \mathrm{MHz}, 410$ $\mathrm{MHz}, 610$ MHz, $1415 \mathrm{MHz}, 2695 \mathrm{MHz}, 4995 \mathrm{MHz}, 8800$ 

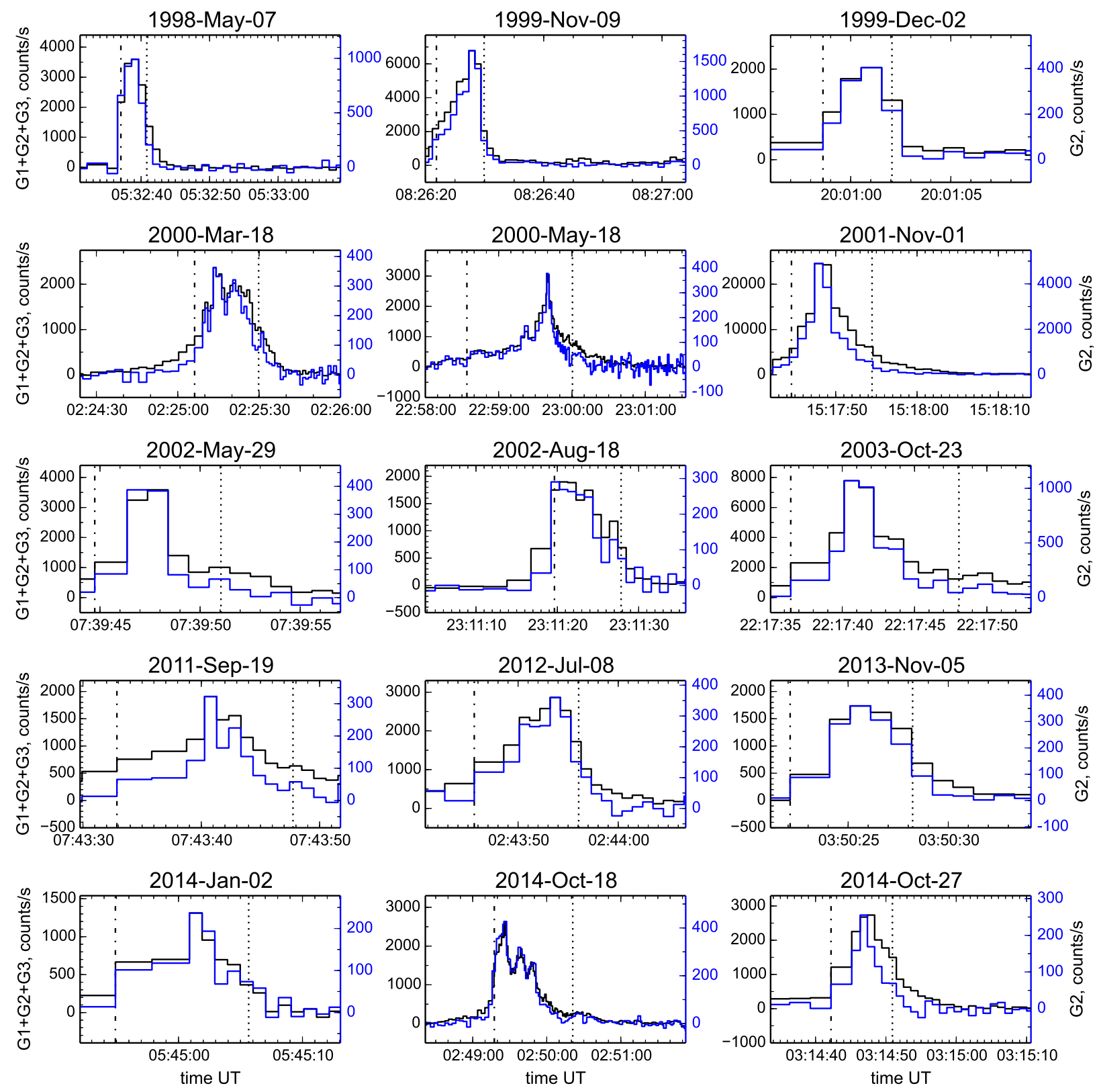

FIG. 1. - CSF time profiles in HXR range measured by Konus- Wind in the sum G1+G2+G3 channels $(\sim 20-1200 \mathrm{keV}$, black curve, left panel) and in G2 channel ( 80-300 keV, blue curve, right panel). For the ease of comparison with Figure 13 here we show 15 cases for which the microwave data allowed spectral fitting.

MHz, $15400 \mathrm{MHz}$ ) from Learmonth, Australia and San Vito, Italy stations.

The OVSA is a solar-dedicated microwave array that consisted of $227-\mathrm{m}$ and $3-52-\mathrm{m}$ antennas at various epoches. The OVSA observed at about 40 frequencies distributed roughly logarithmically between 1.2 and $18 \mathrm{GHz}$. The OVSA observed those 40 frequencies sequentially over $4 \mathrm{~s}$ by small antennas $(8 \mathrm{~s}$ by big antennas), i.e., $0.1 \mathrm{~s}$ per frequency, and can trade-off between the time and spectral resolution depending on the selected observing mode. In a standard mode all frequencies are being observed so the standard time resolution is $4 / 8 \mathrm{~s}$. The OVSA provided total power data in intensity and circular polarizations and offered a limited ability of the source imaging.

NoRP observe the intensity and circular polarization at six frequencies $(1,2,3.75,9.4,17, \& 35 \mathrm{GHz})$ and the intensity only at $80 \mathrm{GHz}$ with the time resolution $0.1 \mathrm{sec}$ in the flare mode, and $1 \mathrm{sec}$ in the background mode (no $80 \mathrm{GHz}$ data).

SRS and BBMS are spectropolarimeters supporting science with Siberian Solar Radio Telescope. SRS measures the integrated flux over the whole solar disk in 2-24 GHz frequency range in two circular polarizations at 16 frequencies with a temporal resolution of $1.6 \mathrm{sec}$ (Muratov 2011). BBMS is the $4-8 \mathrm{GHz}$ spectropolarimeter which measures the integrated flux over the whole solar disk in two circular polarizations at 

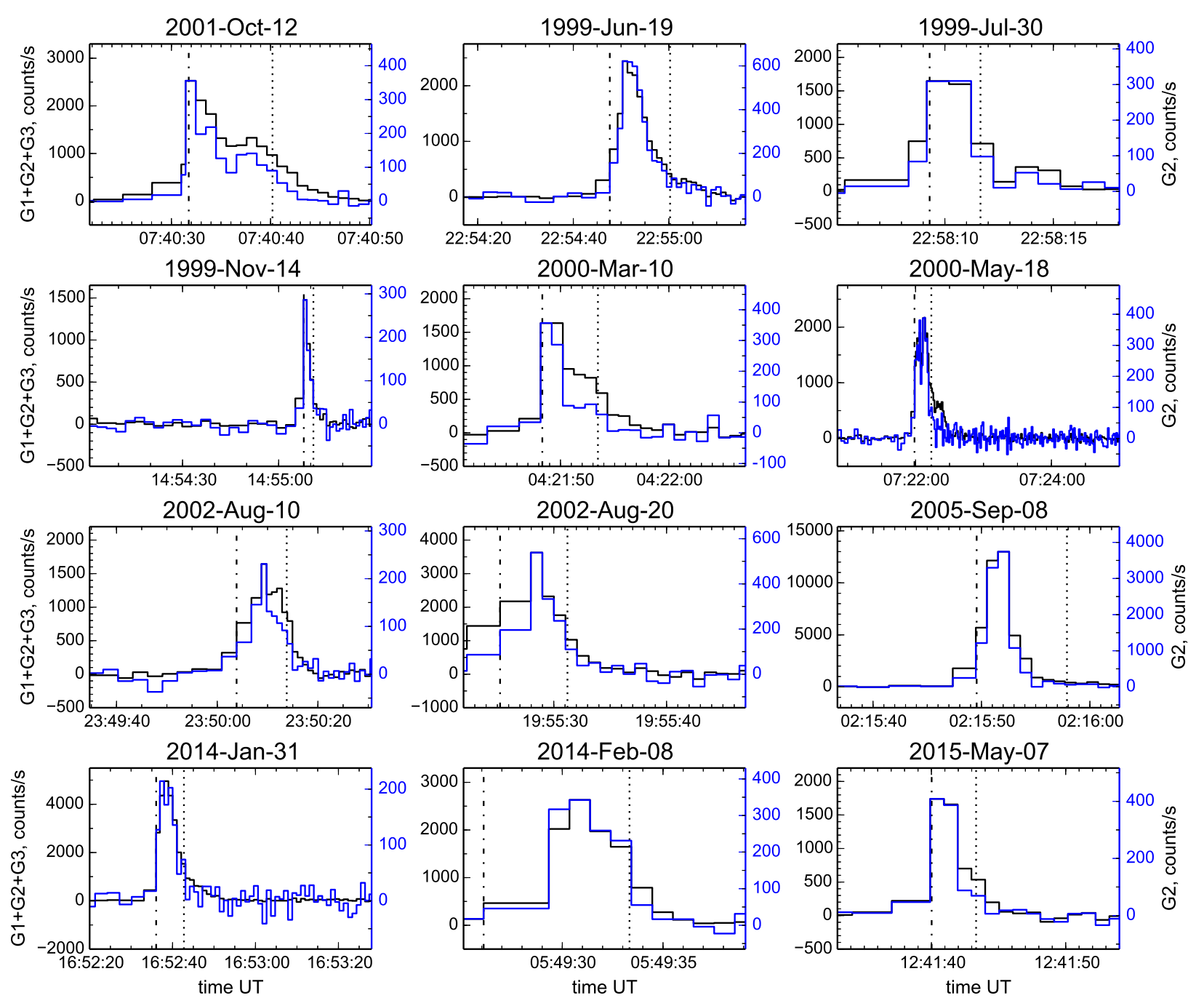

FIG. 2.- CSF time profiles in HXR range measured by Konus- Wind in the sum G1+G2+G3 channels $(\sim 20-1200 \mathrm{keV}$, black curve, left panel) and in G2 channel ( $\sim 80-300 \mathrm{keV}$, blue curve, right panel). For the ease of comparison, here we show 12 cases corresponding to Figure 14

TABLE 2

Specifications of the instruments USED For the MicrowaVE DATABASE.

\begin{tabular}{llcc}
\hline \hline Instr. & \multicolumn{1}{c}{ Frequencies, GHz } & Obs. Time, UT & $\begin{array}{c}\text { Time Res., } \\
\text { High/Low, }\end{array}$ \\
\hline OVSA & $1.2-18 ; 40$ Channels & $\sim 16: 00-24: 00$ & $4 / 8^{*}$ \\
NoRP & $1,2,3.75$, & $\sim 23: 00-07: 00$ & $0.1 / 1$ \\
& $9.4,17,35,80^{* *}$ & & \\
SRS & $2-24 ; 16$ Channels & $\sim 00: 00-10: 00$ & $1.6 / 1.6$ \\
BBMS & $4-8 ; 26$ Channels & $\sim 00: 00-10: 00$ & $0.01 / 0.01$ \\
RSTN & $0.6,1.4,2.7$, & $24 \mathrm{~h}$ & $1 / 1$ \\
& $4.995,8.8,15.4$ & $\sim 08: 00-20: 00$ & $1 / 1$ \\
KMAS & $6.1,9.0$ & & \\
& & &
\end{tabular}

* During dedicated campaigns the the OVSA time resolution was higher at the expense of reducing the number of frequency channels.

** Data at $80 \mathrm{GHz}$ are unavailable in the background mode.

26 frequencies with a temporal resolution of $10 \mathrm{~ms}$. (Zhdanov \& Zandanov 2011).

KMAS measure the integrated solar flux at two fre- quencies, $6.1 \mathrm{GHz}$ and $9.0 \mathrm{GHz}$, with time resolution of 1 s. No polarization measurements is available from KMAS.

\subsubsection{Building the Microwave Burst Database}

The most comprehensive study of the solar microwave burst spectral properties has been performed using the OVSA database accumulated over complete two years of observations during 2001-2002 (Nita et al. 2004), so it would be beneficial to use a similar database here to make fair comparison of our subset of the data with the statistical distributions of all bursts reported by Nita et al. (2004). Unfortunately, only very few events from our list have microwave OVSA data. Nevertheless, we made all possible steps to prepare all available data from other radio instruments in a form as similar as possible to the OVSA data; see Appendix $\mathrm{A}$ for the details. In particular, we combined data obtained by various instruments, fixed clock errors and amplitude calibration errors as well as addressed dissimilar time resolution of the various instruments.

Finally, we built a microwave database composed of the 


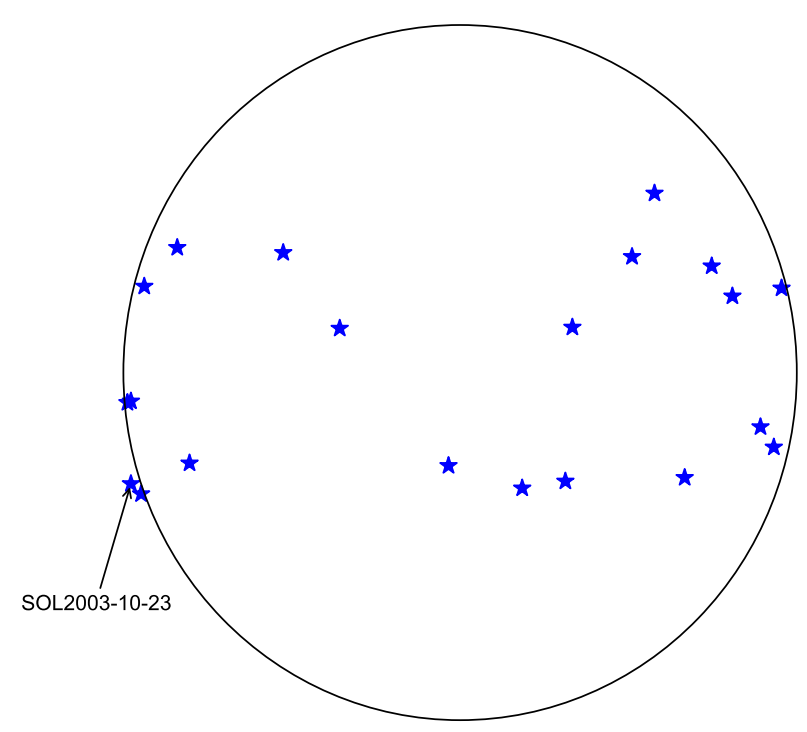

Fig. 3.- CSF positions.

26 events out of the 27 events in the Konus- Wind list; for the remaining event no microwave data were available. Microwave instruments available for each event are listed in Table 6 .

\subsection{Cold Early Impulsive Flare Localization}

We used available imaging information from various instruments to determine flare positions. Whenever available, we used instruments in X-ray and microwave ranges including OVSA, Siberian Solar Radio Telescope (Grechnev et al. 2003), Nobeyama RadioHeliograph (NoRH Nakajima et al. 1994), and Reuven Ramaty High Energy Solar Spectroscopic Imager (RHESSI Lin et al. 2002). For one flare, 1998-May-07, we used brightening in the SoHO/EIT (Domingo et al. 1995) $195 \AA$ data, although this identification may not be reliable, because it corresponds to thermal emission, which is low for CSFs. Eventually, we determined locations of 21 of 27 CSFs, while for the remaining 6 flares no relevant spatial information was found. The heliocentric coordinates of the flares and instruments used for their localization are listed in Table 1. Locations of the flare are also illustrated in Figure 3, which shows that most flares are located on the solar disk, four flares are near the very limb, and one flare, 2003-Oct-23, demonstrates a source above solar limb.

\section{DATA ANALYSIS}

3.1. Relationships between HXR and SXR Emissions and Identification of the Cold Early Impulsive Flares

To compare Konus- Wind and GOES time profiles, corrections for the light propagation time from the Wind spacecraft to the center of the Earth were applied to the Konus- Wind data. Such an approach gives an error within $\sim 20 \mathrm{~ms}$ for the light propagation to any ground- based or earth orbiting instrument, which is a satisfactory accuracy for the presented study.

The duration of the flare impulsive phase was determined in the Konus- Wind G2 channel, which boundaries changed from $\sim 50 \mathrm{keV}-200 \mathrm{keV}$ to $\sim 80 \mathrm{keV}-300 \mathrm{keV}$ during operational history. This channel was selected because it does not contain any contribution from the thermal emission. The Konus- Wind background was approximated by a constant in time range in an interval selected within $-1000 \mathrm{~s}$ and $-200 \mathrm{~s}$ before the flare with fit probability $\geq 5 \%$. The duration of the flare impulsive phase was estimated using the so-called $t_{90}$, which is the difference between $t_{95}$, accumulation time of $95 \%$ of flare integral counts, and $t_{5}$, accumulation time of $5 \%$ of flare integral counts (see Fig 4 ). We employed $t_{90}$ because this value is less sensitive to the choice of signal-to-noise ratio than the total duration, $t_{100}$, (Kouveliotou et al. 1993). Values of $t_{90}$ are listed in Table 3

To quantify the thermal response, which is needed to identify the outliers with a relatively low SXR emission compared to the early impulsive flares showing a more standard heating during the impulsive phase, we measured the increase of the GOES flux at the 1-8 $\AA$ channel ( $\triangle$ GOES) during or following the impulsive HXR emission (see Figure 4). In the case of constant background, the increase of GOES flux in 1-8 $\AA$ channel was obtained as the difference between GOES flux at $t_{95}$ and flux at $t_{5}$. In the case of monotonically varying preflare

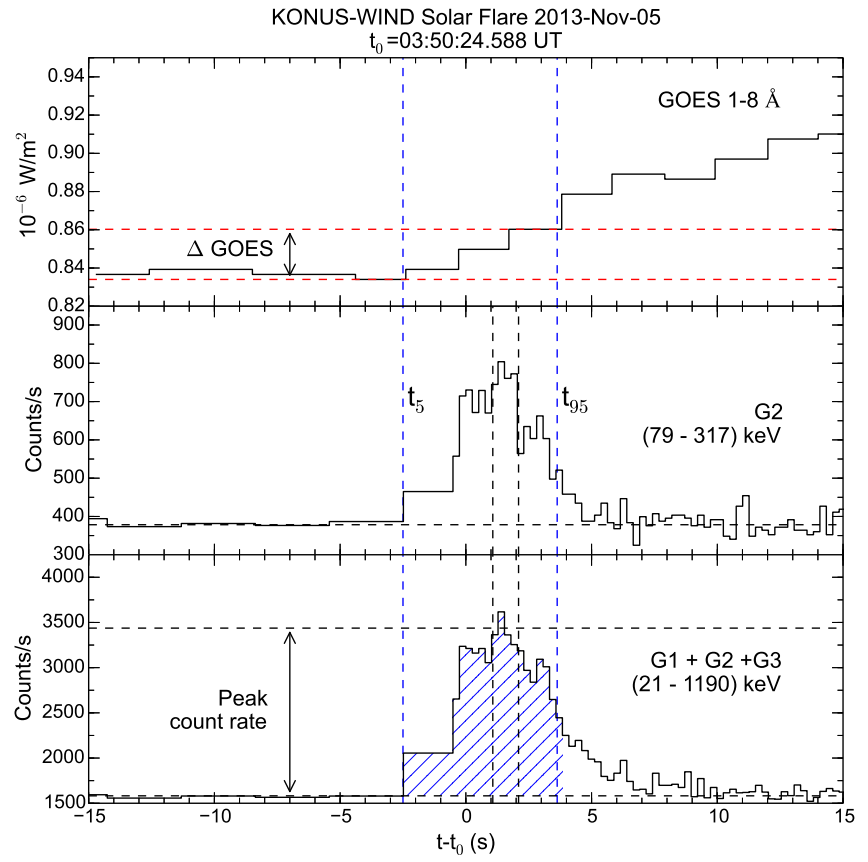

FIG. 4.- Illustration for the measurement of the GOES flux increase, $\triangle$ GOES, during the impulsive HXR emission. $t_{0}$ is the Konus-Wind trigger time after correction for the propagation time from the Wind to the Earth. Vertical dashed blue lines display $t_{90}$, burst duration estimation, determined using G2 Konus- Wind channels, horisontal red dashed lines indicate increase in GOES 1-8 $\AA$ channel during $t_{90}, \Delta$ GOES, black dashed lines refer to peak interval in G2 channel, horisontal black dashed lines display HXR peak count rate determined as the sum of count rates in $\mathrm{G} 1+\mathrm{G} 2+\mathrm{G} 3$ channels, blue hatched area corresponds to HXR integral counts in $\mathrm{G} 1+\mathrm{G} 2+\mathrm{G} 3$ channels accumulated during $t_{90}$ determined by G2 channel. 

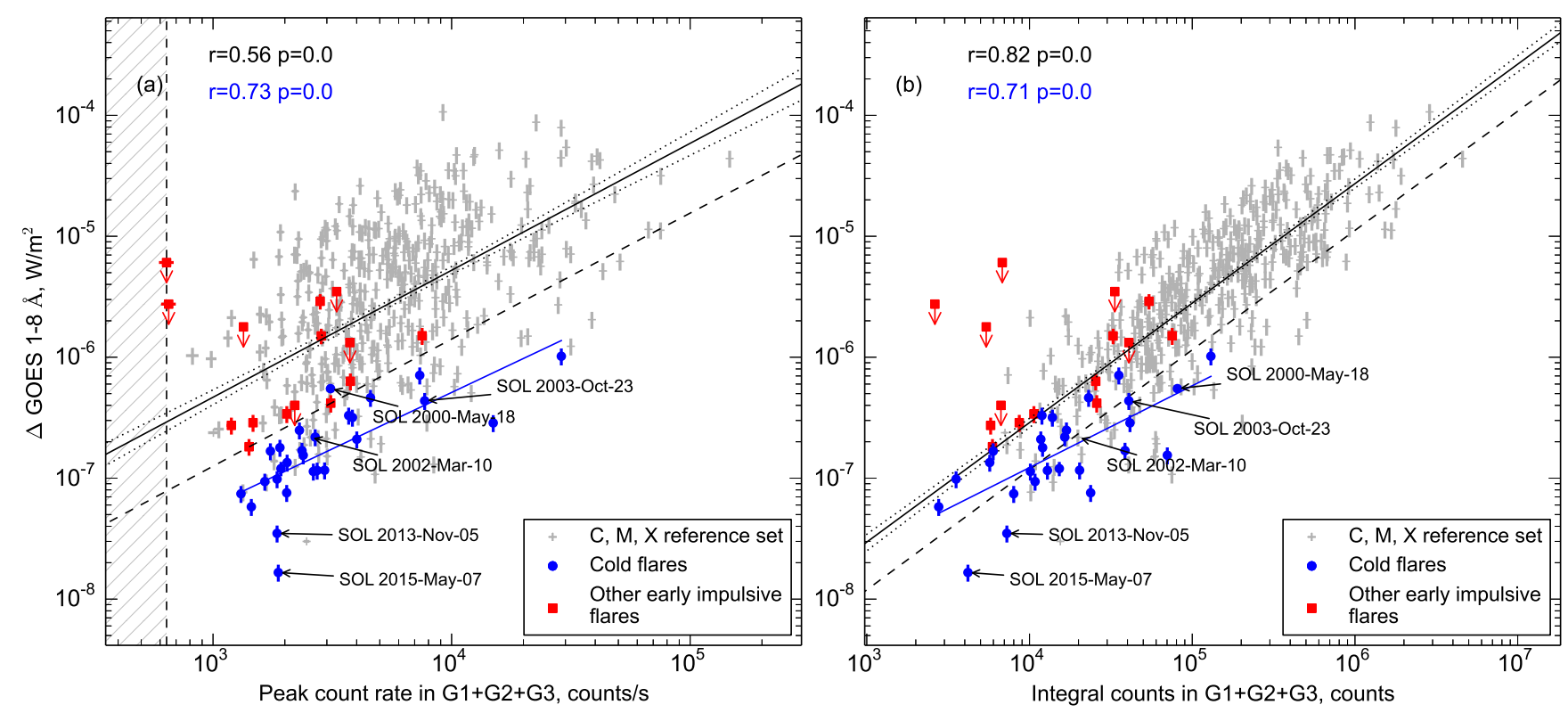

FIG. 5.- Increase in GOES 1-8 $\AA$ channel during impulsive phase of HXR emission vs. (a) HXR peak count rate, (b) HXR integral counts. Black and blue solid lines are linear dependences for all flares (both early impulsive flares and flares from reference set) and CSFs only respectively, black dotted lines display 0.68 confidence band for all flares. Black and blue labels are correlation coefficients (r) and p-values (p) for all flares and for CSFs only correspondingly. Black dashed line represents the bound which separates 'cold' outliers (see the text for more details).

GOES flux, the background was approximated by a 3order polynomial and subtracted, and then the difference between fluxes at $t_{95}$ and $t_{5}$ was calculated. In the case of the absence of observable responce in the GOES 1-8 $\AA$ channel upper limits for $\triangle$ GOES were estimated as $15 \%$ of GOES flux in 1-8 $\AA$ channel which corresponds to GOES error in this channel (Garcia 1994).

The peak time frame was calculated on $1024 \mathrm{~ms}$ timescale according to counts in G2 channel, and the HXR peak count rate was taken as the sum of count rates in $\mathrm{G} 1+\mathrm{G} 2+\mathrm{G} 3$ channels after background substraction (see Figure 4). For one flare, the peak time frame was defined on $2.944 \mathrm{~s}$ timescale because of the failure in G2 trigger time history.

Results for $\triangle$ GOES vs. HXR peak count rate regression for the early impulsive flares and the reference set are shown in Figure 5(a) and are listed in Table 3. The grey hatched area in the left of the figure indicates the feature, that the Konus-Wind cannot register flares with that low HXR peak count rates in the triggered mode. We calculated regression coefficients and confidence interval using python bces Akritas \& Bershady 1996; Nemmen et al. 2012) procedure, which takes into account both $\mathrm{x}$ and y uncertanties, and paired-bootstrap resampling. The black solid line in the Figure corresponds to the linear regression between $\triangle$ GOES and HXR peak count rate for all flares and dotted lines indicate $68 \%$ confidence band. The flares lying near the regression line can be referred as ones with 'mean' thermal response, while those far above the line can be interpreted as thermal dominated, and the flares far below regression line are outliers, which demonstrate lack of thermal emission. The quantitative criterion to select these outliers was as follows. We built distribution of distances between the regression line and each flare with negative distances for the flares below regression line. We considered $68 \%$ of flares, those which lie between 16 and $84 \%$ quantile of distances distribution, have average thermal response, while early impulsive flares below $16 \%$ quantile (dashed line on the Figure) are nonthermal dominated, i. e. 'cold' early impulsive flares.

The blue solid line in the Figure is a rough estimation of the linear regression between $\triangle$ GOES and HXR peak count rate for cold flares only. Several events are individually labeled in this plot: these are flares 2013-Nov-05 and 2015-May-07, which demonstrate very low thermal response, the flare 2002-Mar-10 from Fleishman et al. (2016), and flares 2000-May-18, 22:59 UT, and 2003Oct-23 that will be discussed later. Labels " $r$ " and " $p$ " denote the Pearson correlation coefficient and pvalues (probabilities that correlation between parameters is elusive) for all events (black) and for cold flares only (blue). Correlation coefficients were calculated using python scipy.stats.linregress function between decimal logarithms of observed values hereafter. Correlation coefficient for the cold flares, $r=0.73$, is larger than coefficient for all events is $r=0.56$, $\mathrm{p}$-values are negligible in both cases.

The Konus- Wind HXR integral counts during the impulsive flare phase were estimated as the background subtracted sum of G1+G2+G3 counts during $t_{90}$ defined for G2 channel. Relationship between the HXR integral counts and $\triangle$ GOES is presented in Figure 5(b) and Table [3] As in Figure 5(a), the black and blue solid lines represent linear regressions for all flares and the cold flares, respectively; dotted lines represent $68 \%$ confidence band for all flares, which were obtained similarly to Figure 5(a). Meanings of the labels are the same as those in Figure 5(a). The largest correlation coefficient, $r=0.82$, is for all flares, $r=0.71$ for CSFs, $p$-values for 
TABLE 3

COMPARISON OF COLD SOLAR FLAREPROPERTIES IN HXR AND SXR RANGES.

\begin{tabular}{|c|c|c|c|c|c|c|c|c|}
\hline $\mathrm{N}$ & Date & $t_{0}{ }^{*}, \mathrm{hh}: \mathrm{mm}: \mathrm{ss}$ & $\begin{array}{l}t_{90} \\
(\mathrm{~s})\end{array}$ & $\begin{array}{c}\Delta \mathrm{GOES} \\
10^{-6} \mathrm{Wt} / \mathrm{m}^{2}\end{array}$ & $\begin{array}{l}\text { HXR peak } \\
10^{3} \text { counts } / \mathrm{s}\end{array}$ & $\begin{array}{l}\text { HXR int. } \\
10^{3} \text { counts }\end{array}$ & $\begin{array}{c}\text { HXR peak } \\
10^{-6} \mathrm{erg} /\left(\mathrm{s} \mathrm{cm}^{2}\right)\end{array}$ & $\begin{array}{c}\text { HXR int. } \\
10^{-6} \mathrm{erg} /\left(\mathrm{cm}^{2}\right)\end{array}$ \\
\hline 1 & 1998-Мay-07 & $05: 32: 37.072$ & $3.8 \pm 0.5$ & $0.33 \pm 0.05$ & $3.70 \pm 0.08$ & $11.92 \pm 0.15$ & $6.36 \pm 0.05$ & \\
\hline 2 & 1999-Jun-19 & $22: 54: 49.788$ & $12.6 \pm 1.8$ & $0.25 \pm 0.04$ & $2.30 \pm 0.06$ & $16.87 \pm 0.20$ & $\ldots$ & $18 \pm 3$ \\
\hline 3 & 1999-Jul-30 & $22: 58: 09.675$ & $2.3 \pm 0.3$ & $0.098 \pm 0.016$ & $1.86 \pm 0.06$ & $3.54 \pm 0.09$ & $3.08 \pm 0.07$ & $5.9 \pm 0.9$ \\
\hline 4 & 1999-Nov-09 & $08: 26: 21.703$ & $8.1 \pm 0.4$ & $0.71 \pm 0.11$ & $7.35 \pm 0.09$ & $35.39 \pm 0.22$ & $\ldots$ & $\ldots$ \\
\hline 5 & 1999-Nov-14 & $14: 55: 08.244$ & $3.0 \pm 0.3$ & $0.058 \pm 0.009$ & $1.45 \pm 0.06$ & $2.77 \pm 0.09$ & $2.41 \pm 0.07$ & $4.6 \pm 0.6$ \\
\hline 6 & 1999-Dec-02 & 20:01:00.012 & $3.4 \pm 0.8$ & $0.135 \pm 0.022$ & $2.04 \pm 0.06$ & $5.70 \pm 0.11$ & $3.39 \pm 0.07$ & $6.1 \pm 1.7$ \\
\hline 7 & 2000-Mar-10 & $04: 21: 48.688$ & $5.1 \pm 0.8$ & $0.167 \pm 0.027$ & $1.74 \pm 0.06$ & $5.98 \pm 0.12$ & $2.88 \pm 0.07$ & $10.1 \pm 1.8$ \\
\hline 8 & 2000-Mar-18 & $02: 25: 10.567$ & $23.6 \pm 2.5$ & $0.169 \pm 0.027$ & $2.36 \pm 0.06$ & $38.62 \pm 0.28$ & $3.94 \pm 0.10$ & $57 \pm 10$ \\
\hline 9 & 2000-May-18 & $07: 21: 59.706$ & $14.9 \pm 2.5$ & $0.0757 \pm 0.012$ & $2.04 \pm 0.06$ & $23.75 \pm 0.23$ & $3.37 \pm 0.08$ & $12 \pm 6$ \\
\hline 10 & 2000-May-18 & $22: 59: 39.777$ & $86 \pm 9$ & $0.55^{* * *}$ & $3.11 \pm 0.07$ & $81.0 \pm 0.5$ & $5.26 \pm 0.15$ & $357 \pm 55$ \\
\hline 11 & 2001-Oct-12 & $07: 40: 31.941$ & $8.4 \pm 1.1$ & $0.116 \pm 0.019$ & $2.73 \pm 0.07$ & $12.88 \pm 0.17$ & $4.65 \pm 0.10$ & $22 \pm 3$ \\
\hline 12 & 2001-Nov-01 & $15: 17: 42.772$ & $9.9 \pm 0.5$ & $1.02 \pm 0.16$ & $28.83 \pm 0.17$ & $130.2 \pm 0.4$ & $\ldots$ & $\ldots$ \\
\hline 13 & 2002-May-29 & $07: 39: 46.864$ & $6.3 \pm 1.5$ & $0.21 \pm 0.03$ & $4.00 \pm 0.07$ & $11.73 \pm 0.15$ & $6.82 \pm 0.11$ & $13 \pm 4$ \\
\hline 14 & 2002-Aug-10 & $23: 50: 09.293$ & $9.7 \pm 2.2$ & $0.094 \pm 0.015$ & $1.65 \pm 0.06$ & $10.81 \pm 0.17$ & $\ldots$ & $\ldots$ \\
\hline 15 & 2002-Aug-18 & $23: 11: 19.740$ & $8.2 \pm 1.7$ & $0.179 \pm 0.029$ & $1.91 \pm 0.07$ & $12.03 \pm 0.19$ & $\ldots$ & $\ldots$ \\
\hline 16 & 2002-Aug-20 & $19: 55: 28.476$ & $6.0 \pm 2.1$ & $0.32 \pm 0.05$ & $3.84 \pm 0.08$ & $13.82 \pm 0.18$ & $\ldots$ & $23 \pm 9$ \\
\hline 17 & 2003-Oct-23 & $22: 17: 39.620$ & $11.6 \pm 2.4$ & $0.44 \pm 0.07$ & $7.71 \pm 0.10$ & $40.78 \pm 0.25$ & $\ldots$ & $47 \pm 12$ \\
\hline 18 & 2005-Sep-08 & $02: 15: 49.996$ & $8.3 \pm 2.6$ & $0.29 \pm 0.05$ & $14.94 \pm 0.14$ & $41.44 \pm 0.29$ & $\ldots$ & $72 \pm 22$ \\
\hline 19 & 2011-Sep-19 & $07: 43: 40.791$ & $14.9 \pm 2.8$ & $0.12 \pm 0.02$ & $1.93 \pm 0.06$ & $15.24 \pm 0.20$ & $3.26 \pm 0.07$ & $29 \pm 7$ \\
\hline 20 & 2012-Jul-08 & $02: 43: 50.647$ & $10.4 \pm 2.2$ & $0.116 \pm 0.019$ & $2.94 \pm 0.07$ & $20.32 \pm 0.20$ & $5.05 \pm 0.10$ & $30 \pm 7$ \\
\hline 21 & 2013-Nov-05 & $03: 50: 24.588$ & $6.12 \pm 1.6$ & $0.035 \pm 0.006$ & $1.86 \pm 0.06$ & $7.26 \pm 0.13$ & $3.22 \pm 0.04$ & $5.0 \pm 1.5$ \\
\hline 22 & 2014-Jan-02 & $05: 45: 01.390$ & $10.8 \pm 2.3$ & $0.074 \pm 0.012$ & $1.31 \pm 0.05$ & $7.00 \pm 0.16$ & $2.23 \pm 0.06$ & $13 \pm 5$ \\
\hline 23 & 2014-Jan-31 & $16: 52: 37.461$ & $6.7 \pm 1.1$ & $0.46 \pm 0.07$ & $4.57 \pm 0.08$ & $23.08 \pm 0.18$ & $8.33 \pm 0.29$ & $35 \pm 8$ \\
\hline 24 & 2014-Feb-08 & $05: 49: 29.848$ & $7.2 \pm 2.2$ & $0.114 \pm 0.018$ & $2.63 \pm 0.06$ & $10.12 \pm 0.15$ & $4.48 \pm 0.09$ & $6 \pm 3$ \\
\hline 25 & 2014-Oct-18 & 02:49:17.710 & $64 \pm 10$ & $0.155 \pm 0.025$ & $2.39 \pm 0.04$ & $70.3 \pm 0.4$ & $\ldots$ & $\ldots$ \\
\hline 26 & 2014-Oct-27 & $03: 14: 46.862$ & $8.7 \pm 2.3$ & $0.22 \pm 0.03$ & $2.68 \pm 0.06$ & $16.47 \pm 0.17$ & $4.64 \pm 0.15$ & $35 \pm 11$ \\
\hline 27 & 2015-Мay-07 & $12: 41: 40.415$ & $3.3 \pm 0.7$ & $0.0166 \pm 0.0027$ & $1.88 \pm 0.06$ & $4.18 \pm 0.10$ & $3.27 \pm 0.04$ & $7.2 \pm 1.7$ \\
\hline
\end{tabular}

* The Konus-Wind trigger time after corrections for the light propagation to the Earth are applied.

** Obtained using 3-channel fitting.

*** Upper limits.

these two groups are close to zero. Outliers for this relationship were defined in the same manner as for $\triangle \mathrm{GOES}$ vs. HXR peak count rate relationship. For the most cases, the 'cold' early impulsive outliers in Figure 5(b) are also the outliers in panel (a). The only exception is the event of 2000-May-18, 22:59 UT.

As the Konus-Wind energy boundaries changed with time it is reasonable to compare the relationships obtained from the instrumental HXR characteristics, such as HXR peak count rate and HXR integral counts, with those obtained from unfolded characteristics, namely HXR peak flux and HXR integral flux. To find HXR fluxes in physical units (for example, erg $\mathrm{s}^{-1} \mathrm{~cm}^{-2}$ ) especially in the case of rather broad energy channels, a spectral model should be selected, then spectral fitting performed, and HXR fluxes calculated based on the obtained fitting parameters. To keep uniformity of the fitting results, 3-channel fitting rather than multichannel one was employed, because a fraction of the flare impulsive phase might have occurred before the Konus-Wind trigger, i.e. in the waiting mode data, where no multichannel spectra are available.

Results of 3-channel and multichannel fitting with power-law model were compared for peak spectra of early impulsive flares and some reference flares (see Section 3.2). Results with fit probabilities $<1 \%$ were neglected. Comparisons between the results of the 3channel and multichannel fitting are presented in Figure 6] namely, comparison between the power-law indices (left) and between HXR fluxes in 20-1000 keV range (right). Errors on both plots refer to $68 \%$ confidence level. Dashed line indicates the expected equality of fit results, while the solid line refers to the linear regression between multichannel and 3-channel fit results. As can be seen from Figure 6, the results of multichannel and 3channel fitting are in good agreement with the exception of one event. Thus, we do not apply any extra corrections to 3-channel fitting results.

Relationships between $\Delta$ GOES and the HXR peak flux or the integral flux are presented in Figure 7 and Table 3. Meanings of labels and lines are the same as in Figure 5(a). From comparison between Figure 5(a) and Figure 7 (a) it is clear that the same flares form the set of 'cold flare' outliers in both plots. Thus, the Konus- Wind energy boundary variations do not significantly affect our selection of the CSFs. The values of the correlation coefficients for regressions shown in Figures [5(a) and 7 (a) are also close to each other; specifically, for all flares: $r=0.60$ for the HXR peak flux vs $r=0.56$ for the HXR peak count rate, $\mathrm{p}$-value is also negligible and for the CSFs: $r=0.68$ vs $r=0.73$ and $\mathrm{p}$-value is $1.9 \mathrm{e}-3$.

The situation is similar comparing Figures 5(b) and Figures 7(b): the correlation coefficient for all flares remains $r=0.83$ and the CSFs correlation coefficient also didn't change significantly and probabilities, that correlations are elusive, are also close to zore for all three groups. But some CSFs move closer to the main regression line comparing to relation between $\triangle \mathrm{GOES}$ and HXR integral counts. This can be caused by underestimation of integral flux while averaging flux over flare duration in the fitting procedure.

Thus for flares, for which unfolded HXR fluxes were ob- 

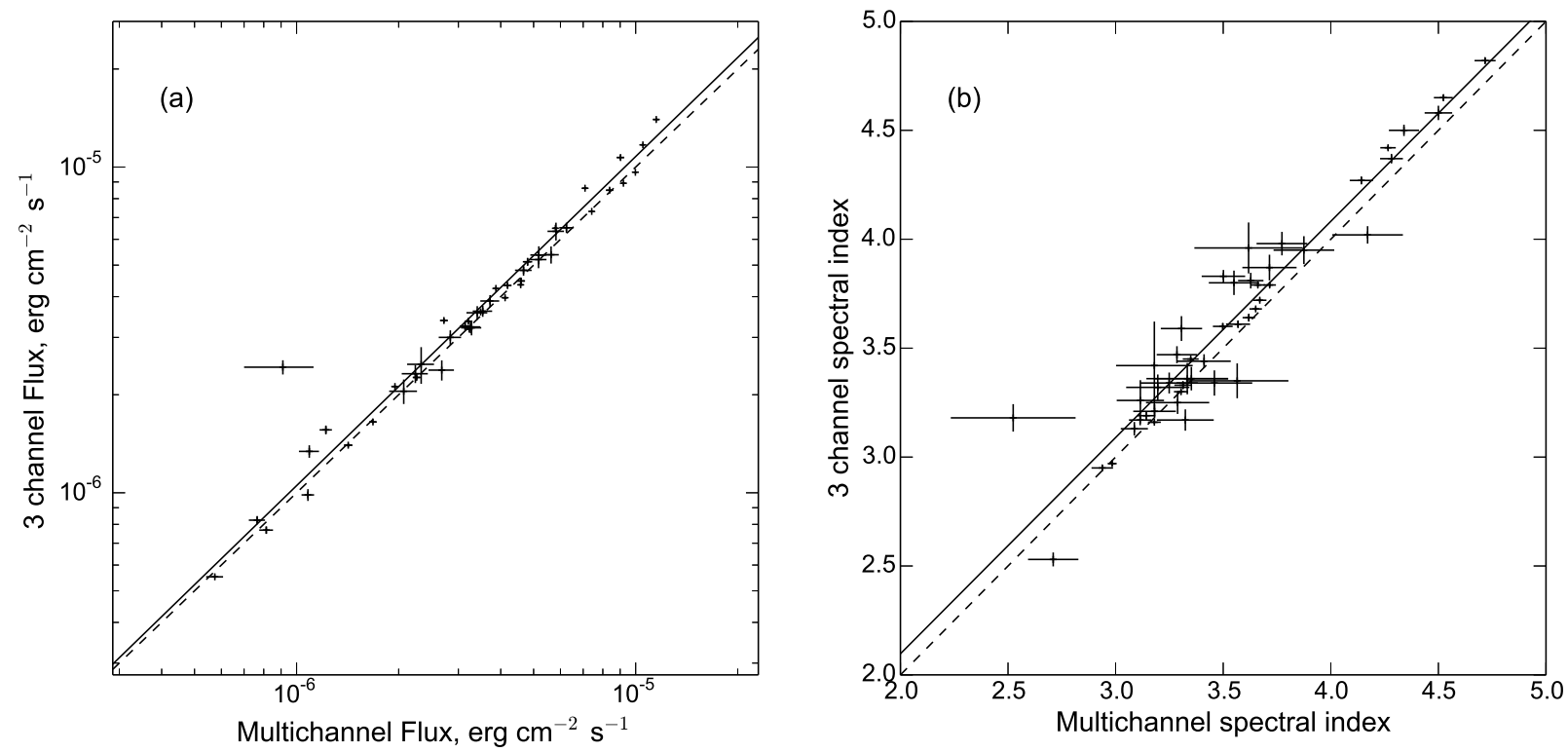

FIG. 6.- Comparison between results of multichannel fitting and 3-channel fitting with power law model (a) for fluxes, (b) for spectral indices. Fitting was held on time frames corresponding to the accumulation time of peak multichannel spectra. Results with fit probability less than 0.01 were neglected. The dashed line reflects the equality and the solid line indicates linear regression between 3-channel and multichannel results.
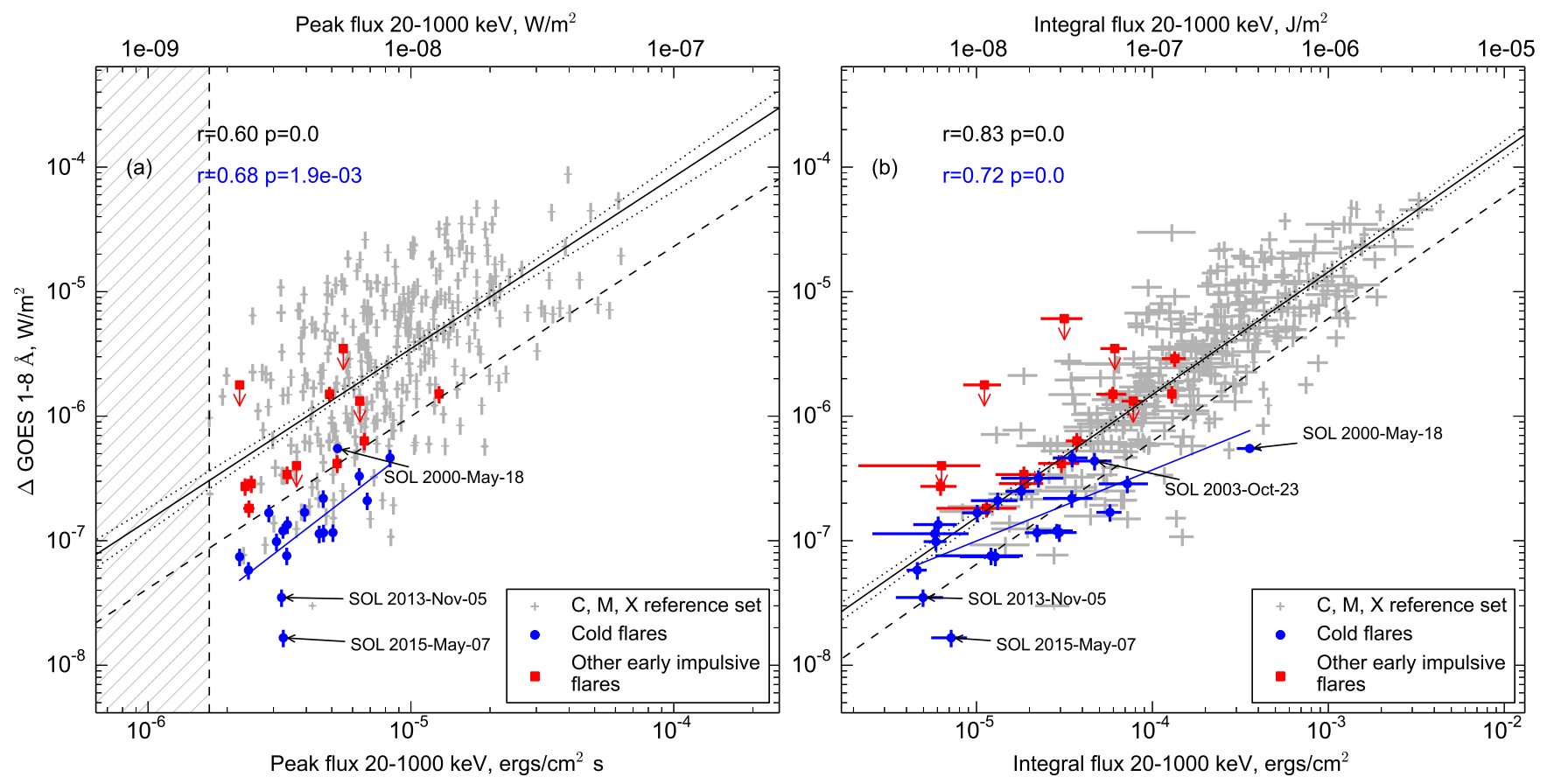

FIG. 7.- Increase in GOES 1-8 $\AA$ channel during impulsive phase of HXR emission vs. (a) HXR peak flux, (b) HXR integral flux. Black and blue solid lines are linear dependences for all flares (both early impulsive flares and flares from reference set) and CSFs only respectively, black dotted lines display 0.68 confidence band for all flares. Black and blue labels are correlation coefficients (r) and p-values (p) for all flares and for CSFs only corresponingly. Black dashed line represents the bound which separates 'cold' outliers (see the text for more details). 


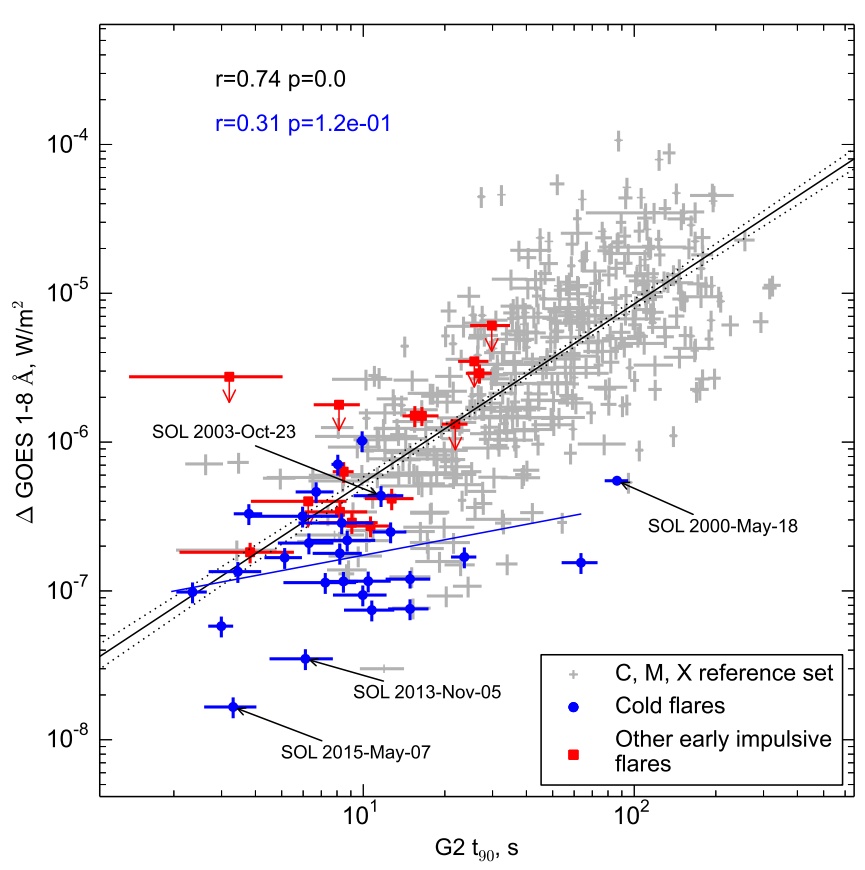

FIG. 8.- Increase in GOES 1-8 $\AA$ channel during impulsive phase of HXR emission vs. $t_{90}$. Black and blue solid lines are linear dependences for all flares (both early impulsive flares and flares from reference set) and cold flares respectively, black dotted lines display 0.68 confidence band for all flares. Black and blue labels are correlation coefficients (r) and p-values (p) for all flares and for cold flares corresponingly.

tained, we conclude that the Konus- Wind energy boundaries evolution does not affect the proportions between HXR and SXR characteristics considerably. So, for selection of CSF group we formulate the criterion based on instrumental HXR estimations: at least one of the following statements must hold for a flare to be classified as "cold" - either (1) the ratio between $\triangle$ GOES and HXR peak flux or (2) the ratio between $\Delta$ GOES and HXR integral counts is lower than that for the majority $(84 \%)$ of flares. This approach is more universal than using the unfolded data, because these observational measures can be obtained for all events. This approach yielded 27 CSFs listed in Table 1 on which we focus in this paper.

Relationship between increase in GOES channel 1-8 $\AA$ and $t_{90}$ is plotted in Figure 8 . As expected, a high correlation between $\triangle$ GOES and $t_{90}$ is observed for all flares $r=0.74$. CSFs are grouped in the area of low $\Delta$ GOES and low $t_{90}$ excepting two events and thus do not demonstrate significant correlation, $r=0.31$.

\subsection{Spectral properties of the HXR bursts}

The HXR spectral analysis was perfomed using the Konus- Wind multichannel data for 25 of 27 CSFs, because for 2 event spectral data were damaged. In addition, multichannel fits were obtained for 71 events from non-cold-flare reference set occurred during years 2010 2016.

The HXR spectrum fitting was performed on the peak spectra; the peaks were defined according to the count rates in G2 channel (see Section 3.1). The photon energy range between 20 and $1000 \mathrm{keV}$ was considered for analysis of both cold and reference flares: $20 \mathrm{keV}$ corresponds to the low-energy boundary of Konus-Wind, while no photons above $1000 \mathrm{keV}$ were considered, even when present, because the nuclear deexcitation line emission may contribute to the spectrum at those high energies in addition to the nonthermal electron bremsstrahlung. The spectral channels were grouped to have a minimum of 10 counts per channel to ensure the validity of the $\chi^{2}$ statistic. The spectral analysis was performed using XSPEC 12.9.0 (Arnaud 1996).

Initially, we attempted fitting the spectra using the single power-law (PL) model, but the peak spectra were inconsistent with this simple model for many flares because of a spectral break. Thus, for spectra inconsistent with PL we used the phenomenological broken power-law model, 2PL:

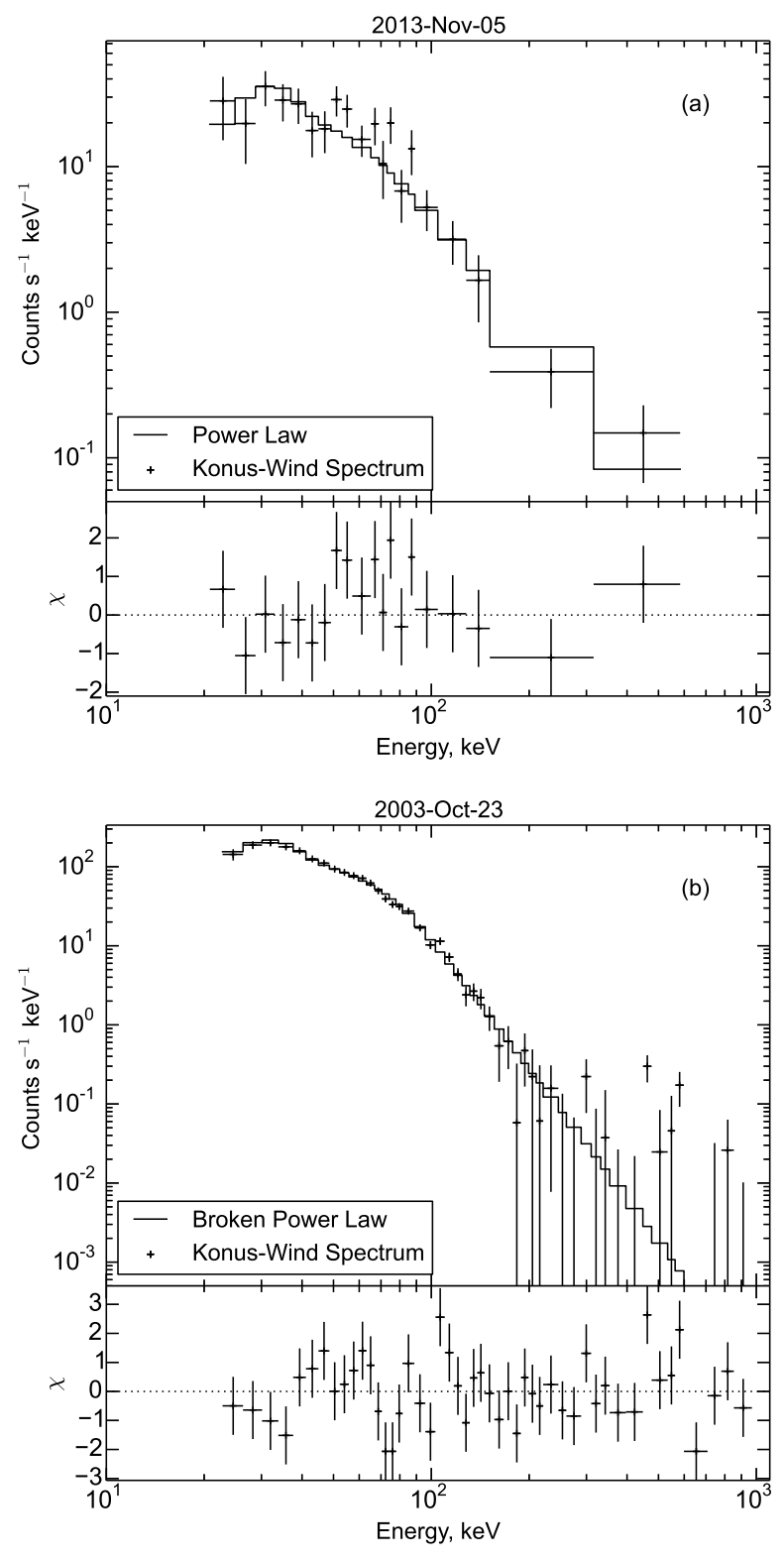

FIG. 9.- Examples of HXR CSFs spectra fittes by (a) PL and (b) 2PL models. 
TABLE 4

PL AND 2PL FIT RESUlts.

\begin{tabular}{|c|c|c|c|c|c|c|c|c|}
\hline $\mathrm{N}$ & Date & $t_{0}{ }^{*}, \mathrm{hh}: \mathrm{mm}: \mathrm{ss}$ & $\gamma_{1}$ & $\begin{array}{l}E_{\text {break,ph }} \\
\quad \text { keV }\end{array}$ & $\gamma_{2}$ & $\begin{array}{c}\text { Photon flux } \\
10^{-6} \mathrm{erg} /\left(\mathrm{s} \mathrm{cm}^{2}\right)\end{array}$ & $\chi^{2} /$ dof & Prob. \\
\hline 1 & 1998-Мay-07 & $05: 32: 37.072$ & $2.51_{-0.12}^{+0.11}$ & $67_{-6}^{+6}$ & $3.67_{-0.18}^{+0.22}$ & $4.76_{-0.16}^{+0.16}$ & $52.53 / 57$ & $6.4 \mathrm{e}-01$ \\
\hline 2 & 1999-Jun-19 & $22: 54: 49.788$ & $2.68_{-0.08}^{+0.09}$ & $101_{-11}^{+19}$ & $\begin{array}{l}4.5_{-0.4}^{+0.9} \\
\text { +o }\end{array}$ & $2.76_{-0.07}^{+0.07}$ & $57.10 / 57$ & $4.7 \mathrm{e}-01$ \\
\hline 3 & 1999-Jul-30 & 22:58:09.675 & $3.26_{-0.15}^{+0.17}$ & $\ldots$ & $\ldots$ & $2.81_{-0.21}^{+0.21}$ & $11.27 / 26$ & $9.9 \mathrm{e}-01$ \\
\hline 4 & 1999-Nov-09 & $08: 26: 21.703$ & $2.49_{-0.13}^{+0.13}$ & $66_{-6}^{+8}$ & $3.86_{-0.12}^{+0.18}$ & $10.75_{-0.27}^{+0.28}$ & $56.57 / 41$ & $5.3 \mathrm{e}-02$ \\
\hline 5 & 1999-Nov-14 & $14: 55: 08.244$ & $2.52_{-0.23}^{+0.29}$ & $\ldots$ & $\ldots$ & $0.91_{-0.21}^{+0.21}$ & $24.12 / 26$ & $5.7 \mathrm{e}-01$ \\
\hline 6 & 1999-Dec-02 & 20:01:00.012 & $3.46_{-0.16}^{+0.18}$ & $\ldots$ & $\ldots$ & $2.84_{-0.21}^{+0.21}$ & $23.47 / 25$ & $5.5 \mathrm{e}-01$ \\
\hline 7 & 2000-Mar-10 & $04: 21: 48.688$ & $3.29_{-0.14}^{+0.15}$ & $\ldots$ & $\ldots$ & $3.26_{-0.22}^{+0.22}$ & $28.00 / 26$ & $3.6 \mathrm{e}-01$ \\
\hline 8 & 2000-Mar-18 & $02: 25: 10.567$ & $3.17_{-0.14}^{+0.22}$ & $60_{-5}^{+16}$ & $4.7_{-0.2}^{+0.9}$ & $2.88_{-0.11}^{+0.17}$ & $50.41 / 57$ & $7.2 \mathrm{e}-01$ \\
\hline 9 & 2000-May-18 & $07: 21: 59.706$ & $3.13_{-0.27}^{+0.09}$ & $72_{-15}^{+6}$ & $5.1_{-0.7}^{+0.5}$ & $2.74_{-0.15}^{+0.09}$ & $78.38 / 58$ & $3.9 \mathrm{e}-02$ \\
\hline 10 & 2000-May-18 & $22: 59: 39.777$ & $3.77_{-0.11}^{+0.12}$ & $\ldots$ & $\ldots$ & $5.6_{-0.3}^{+0.3}$ & $30.34 / 25$ & $2.1 \mathrm{e}-01$ \\
\hline 11 & 2001-Oct-12 & $07: 40: 31.941$ & $3.12_{-0.10}^{+0.11}$ & $\ldots$ & $\ldots$ & $4.67_{-0.26}^{+0.26}$ & $35.23 / 27$ & $1.3 \mathrm{e}-01$ \\
\hline 12 & 2001-Nov-01 & $15: 17: 42.772$ & & $\ldots$ & $\ldots$ & -0.20 & $\ldots$ & $\ldots$ \\
\hline 13 & 2002-May-29 & $07: 39: 46.864$ & $3.29_{-0.09}^{+0.09}$ & $\ldots$ & $\ldots$ & $6.3_{-0.3}^{+0.3}$ & $29.83 / 25$ & $2.3 \mathrm{e}-01$ \\
\hline 14 & 2002-Aug-10 & 23:50:09.293 & $\ldots$ & $\ldots$ & $\cdots$ & $\ldots$ & $\ldots$ & $\ldots$ \\
\hline 15 & 2002-Aug-18 & $23: 11: 19.740$ & $3.17_{-0.17}^{+0.18}$ & $\ldots$ & $\ldots$ & $2.66_{-0.25}^{+0.25}$ & $31.69 / 26$ & $2.0 \mathrm{e}-01$ \\
\hline 16 & 2002-Aug-20 & $19: 55: 28.476$ & $2.30_{-0.26}^{+0.24}$ & $64_{-8}^{+12}$ & $4.2_{-0.4}^{+0.7}$ & $6.2_{-0.4}^{+0.4}$ & $45.69 / 31$ & $4.3 \mathrm{e}-02$ \\
\hline 17 & 2003-Oct-23 & $22: 17: 39.620$ & $2.94_{-0.08}^{+0.07}$ & $74_{-5}^{+4}$ & $5.12_{-0.27}^{+0.28}$ & $11.53_{-0.27}^{+0.28}$ & $57.63 / 44$ & $8.2 \mathrm{e}-02$ \\
\hline 18 & 2005-Sep-08 & $02: 15: 49.996$ & $2.08_{-0.11}^{+0.07}$ & $106_{-20}^{+15}$ & $3.27_{-0.24}^{+0.26}$ & $16.9_{-0.5}^{+0.5}$ & $80.94 / 53$ & $8.0 \mathrm{e}-03$ \\
\hline 19 & 2011-Sep-19 & $07: 43: 40.791$ & $3.20_{-0.14}^{+0.15}$ & $\ldots$ & $\ldots$ & $3.28_{-0.22}^{+0.22}$ & $18.17 / 23$ & $7.5 \mathrm{e}-01$ \\
\hline 20 & 2012-Jul-08 & $02: 43: 50.647$ & $3.31_{-0.13}^{+0.14}$ & $\ldots$ & $\ldots$ & $3.81_{-0.25}^{+0.25}$ & $47.16 / 25$ & $4.7 \mathrm{e}-03$ \\
\hline 21 & 2013-Nov-05 & $03: 50: 24.588$ & $2.63_{-0.12}^{+0.13}$ & $\ldots$ & $\ldots$ & $2.87_{-0.23}^{+0.23}$ & $26.50 / 24$ & $3.3 \mathrm{e}-01$ \\
\hline 22 & 2014-Jan-02 & 05:45:01.390 & $3.18_{-0.16}^{+0.18}$ & $\ldots$ & $\ldots$ & $2.07_{-0.20}^{+0.20}$ & $32.27 / 22$ & $7.3 \mathrm{e}-02$ \\
\hline 23 & 2014-Jan-31 & $16: 52: 37.461$ & $4.17_{-0.11}^{+0.11}$ & $\ldots$ & $\ldots$ & $7.8_{-0.4}^{+0.4}$ & $44.75 / 21$ & $1.9 \mathrm{e}-03$ \\
\hline 24 & 2014-Feb-08 & $05: 49: 29.848$ & $3.32_{-0.12}^{+0.13}$ & $\ldots$ & $\ldots$ & $3.54_{-0.23}^{+0.23}$ & $36.4 / 24$ & $5.0 \mathrm{e}-02$ \\
\hline 25 & 2014-Oct-18 & 02:49:17.710 & & $\cdots$ & $\cdots$ & & $\ldots$ & $\ldots$ \\
\hline 26 & 2014-Oct-27 & $03: 14: 46.862$ & $3.32_{-0.22}^{+0.24}$ & $\ldots$ & $\ldots$ & $2.14_{-0.26}^{+0.26}$ & $16.99 / 22$ & $7.6 \mathrm{e}-01$ \\
\hline 27 & 2015-May-07 & $12: 41: 40.415$ & $2.73_{-0.14}^{+0.15}$ & $\cdots$ & $\cdots$ & $2.61_{-0.24}^{+0.24}$ & $29.40 / 27$ & $3.4 \mathrm{e}-01$ \\
\hline
\end{tabular}

The Konus- Wind trigger time after corrections for the light propagation to the Earth are applied.

$$
I(E)= \begin{cases}A\left(\frac{E}{100 k e V}\right)^{-\gamma_{1}} & E \leq E_{\text {break }, p h} \\ A E_{\text {break,ph }}^{\gamma_{2}-\gamma_{1}}\left(\frac{E}{100 k e V}\right)^{-\gamma_{2}} & E_{\text {break }, p h}<E\end{cases}
$$

where $A$ is the normalization at $100 \mathrm{keV}$ in units of photons $\mathrm{cm}^{-2} \mathrm{~s}^{-1} \mathrm{keV}^{-1}$. Photon flux was calculated in 20-1000 keV range using cflux convolution model in XSPEC assuming spectrum integration within 20 and $1000 \mathrm{keV}$.

In cases when both PL and 2PL models were consistent with the data, the preferred model was chosen according to $F$-test (Bevington 1969), i.e. the decrease in $\chi^{2}$ vs. the degrees of freedom decrease: the criterion for accepting $2 \mathrm{PL}$ model (a model with 2 additional free parameters compared to the PL model) was a decrease of $\chi^{2}>13.5$ using $2 \mathrm{PL}$ model compared to using $\mathrm{PL}$ model which corresponds to chance probability of such decrease $\mathrm{p} \leq 0.1 \%$. One CSFs and 3 flares from the reference group with fit probabilities for both PL and $2 \mathrm{PL}$ model $\mathrm{p} \leq 0.1 \%$ were excluded.

Examples of CSFs spectra fitted by PL and 2PL models are presented in Figure 9 and the fit results for all CSFs with successful fits are listed in Table 4. The PL model successfully fits 16 of $25 \mathrm{CSFs}$ and 25 of 71 reference flares. Distributions of obtained fitting parameters $\gamma$ and photon flux are presented in Figure 10(a-b). On each plot, the median values and $50 \%$ ranges are marked for cold flares (blue) and reference flares (grey), the value " $p$ " denotes probability obtained by the KolmogorovSmirnov test hereafter in the assumption that distributions for cold flares and reference set are similar. The Kolmogorov-Smirnov test was performed using python scipy.stats.ks_2samp function. The median values of the photon spectral indices $\gamma$ for the CSFs and reference flares are equal to 3.3 and 3.5, respectively; though the difference between the median values is not high, but $50 \%$ range is narrower for CSFs and $\gamma$ distribution is shifted towards lower values, thus CSFs are significantly harder (probability that two distributions are equal is $\sim 1 \%$ ). The median value of the photon flux for CSFs is almost two times lower than for the reference set, $3.1 \times 10^{-6}$ vs. $5.8 \times 10^{-6}$ erg $\mathrm{cm}^{2} \mathrm{~s}^{-1}$; there are no events with peak fluxes greater than $10.0 \times 10^{-6}$ erg $\mathrm{cm}^{2} \mathrm{~s}^{-1}$ among CSFs, while the peak fluxes for reference flares extend up to $40.0 \times 10^{-6} \mathrm{erg} \mathrm{cm}^{2} \mathrm{~s}^{-1}$, p-value is also low, $0.75 \%$.

Fitting using the 2PL model was successful for 8 CSFs and 43 flares from the reference set. This means that the $2 \mathrm{PL}$ model was required much more rarely for CSFs than for the reference flares. This might happen, at least for a fraction of flares, because the CSFs are lower intensity flares, for which low signal-to-noise ratio in higher energy range did not allow to detect the energy break even if existed. Distributions of the fitting parameters $\gamma_{1}, E_{\text {break,ph }}, \gamma_{2}$ and photon flux for $2 \mathrm{PL}$ are plotted 

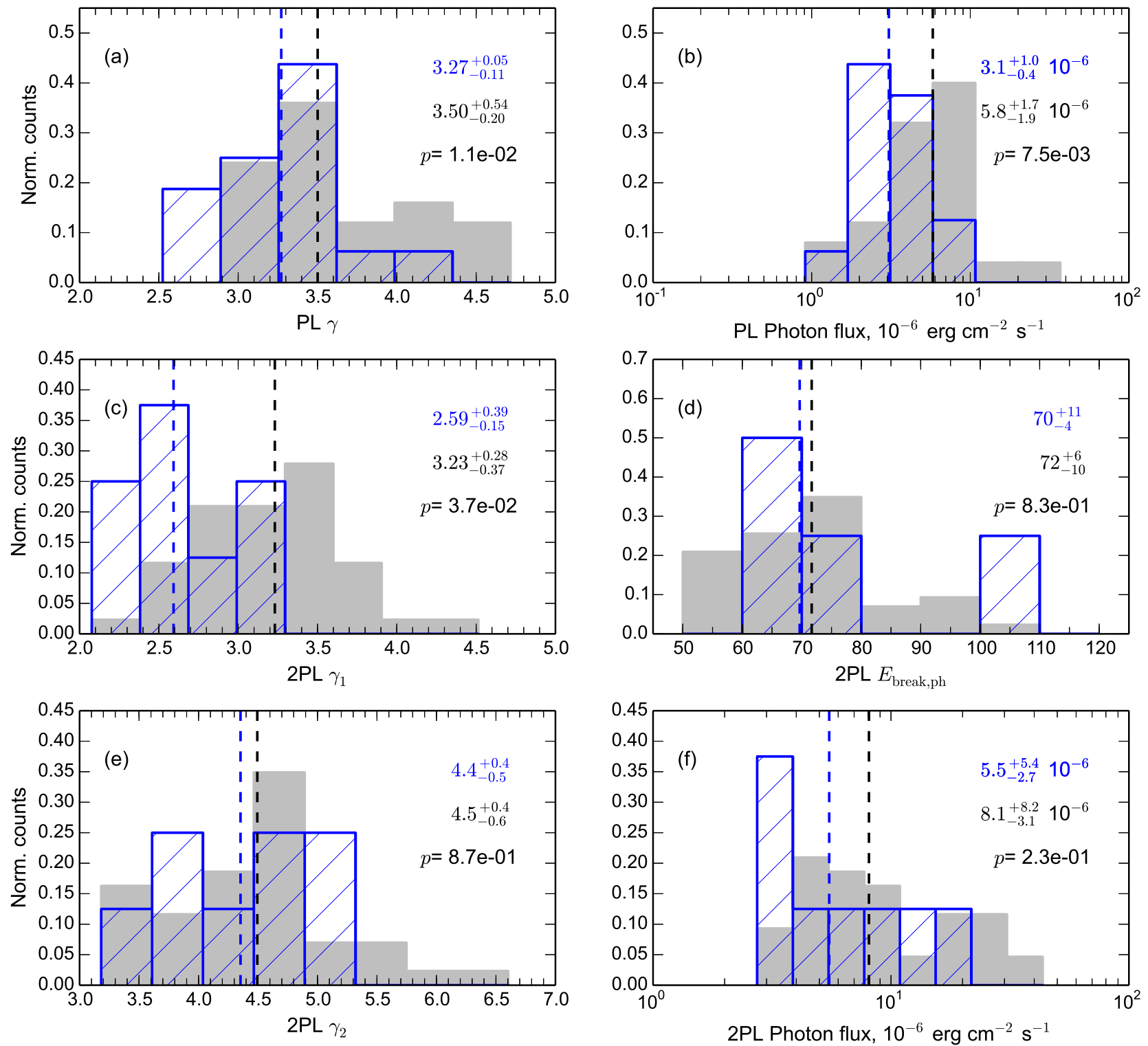

FIG. 10.- Parameter distributions for HXR photon spectra. Bin heights are normalized to total number of events in each group. Blue hatched histograms refer to cold flares, grey histograms - to reference group. Median values and 0.5 probability ranges are presented on each plot for cold flares and for reference group in blue and black letters respectively. Black "p" indicates two-sided p-value from Kolmogorov-Smirnov test in suggestion that distributions of a given parameter for cold flares and reference flares are equal. (a) PL photon spectral index, $\gamma$, distributions; (b) PL photon flux distributions in 20-1000 keV range; (c) 2PL photon spectral index in lower energy range, $\gamma_{1}$, distributions; (d) $2 \mathrm{PL}$ break energy of photon spectrum, $\mathrm{E}_{\text {break,ph }}$, distributions; (e) $2 \mathrm{PL}$ photon spectral index in higher energy range, $\gamma_{2}$, distributions; (f) $2 \mathrm{PL}$ photon flux distributions in 20-1000 keV range.

in Figure 10(c-f). Distributions of low energy photon power-law index $\gamma_{1}$ show that for 2PL model CSFs are also harder: the median value for CSFs is 2.6 vs. 3.2 for the reference group and the overall shape of the histogram is shifted towards lower values of $\gamma_{1}$ as compared to the reference flares, $\mathrm{p}$-value is $p=3.7 \%$. The median values of photon break energies $E_{\text {break,ph }}$ for both groups are close to $70 \mathrm{keV}$. For CSFs there are no events with breaks below $60 \mathrm{keV}$ and for the reference group there are no events with break below $50 \mathrm{keV}$. Probability of $E_{\text {break,ph }}$ distributions coincidence for CSFs and reference set is $83 \%$. The median value of the high-energy photon power-law index, $\gamma_{2}$, for CSFs is also a slightly harder than for the reference set, 4.4 vs. 4.5 , but this difference is insignificant, the probability that $\gamma_{2}$ distributions for CSFs and reference set distributions are equal, is very high: $87 \%$. Photon flux distributions are presented in Figure 10(f), these values may differ from those listed in Table 3. because they were obtained on a different timescales. The median value for the reference set is $8.1 \times 10^{-6} \mathrm{erg} \mathrm{cm}^{-2} \mathrm{~s}^{-1}$ which is higher than for CSFs, $5.5 \times 10^{-6}$ erg $\mathrm{cm}^{-2} \mathrm{~s}^{-1}$, but also it could be insignificant, $p=23 \%$. For both CSFs and reference flares $\gamma_{2}$ is always larger (by absolute value) than $\gamma_{1}$; thus, no break-ups were observed in the entire analyzed data set. A likely reason for this finding, that is in apparent con- 
trast with many reports of HXR spectra with break-ups, is the trigger nature of Konus-Wind that only records flares with reasonably hard spectra, while the break-ups are typically observed when the low-energy part of the HXR spectrum is steep.

To recover spectrum of accelerated electrons from photon spectrum we used collisional thick-target model (Brown 1971) with power-law spectrum of nonthermal electrons (brmThickPL):

$$
F(E)= \begin{cases}0 & E<E_{\text {cut }, \text { low }} \\ \propto A E^{-\delta} & E_{\text {cut }, \text { low }} \leq E \leq E_{\text {cut }, \text { high }} \\ 0 & E_{\text {cut }, \text { high }}<E\end{cases}
$$

where $A$ is the electron flux in electron $\mathrm{keV}^{-1} \mathrm{~s}^{-1}$. This model gives a simple relationship between the electron and photon power-law indices $\gamma=\delta-1$ (Brown 1971; Somov \& Syrovatskii 1976).

For a fraction of both CSFs and reference flares the spectral break is observed and brmThickPL model is not applicable in these cases. In principle, some spectral flattening may be caused by instrumental pile-up effect, thus we performed the Monte-Carlo modeling which revealed that for the Konus-Wind spectra this effect becomes significant for count rates $\geq 5 \times 10^{4}$ counts $/ \mathrm{s}$ (deadtime corrected), but among both CSFs and reference flares used for multichannel fitting there are no such intense events. A spectral flattening at low energies may also be induced by several physical effects: photospheric albedo (Kontar et al. 2006), non-uniform target ionization (Holman et al. 2011) and energy losses associated with a return current (Holman et al. 2011). These effects give spectral breaks at lower energies of the HXR nonthermal spectra, while $E_{\text {break,ph }} \geq 60 \mathrm{keV}$ for all CSFs. Thus, we conclude that spectral flattening at low energies is caused by non-power-law spectrum of accelerated electrons and in addition to brmThickPL model we use collisional thick-target model with broken power-law spectum of nonthermal electrons (brmThick2PL):

$$
F(E)= \begin{cases}0 & E<E_{\text {cut }, \text { low }} \\ \propto E^{-\delta_{1}} & E_{\text {cut }, \text { low } \leq E \leq E_{\text {break,el }} \leq E} \\ \propto E^{-\delta_{2}} & E_{\text {br,e }} \leq E \leq E_{\text {cut }, \text { high }} \\ 0 & E>E_{\text {cut }, \text { high }}\end{cases}
$$

To minimize potential contributions from photospheric albedo, non-uniform target ionization, and return current to the spectrum formation of reference flares we excluded 9 flares from the reference group where $E_{\text {break,ph }} \leq 60 \mathrm{keV}$. To fairly compare the electron fluxes in all cases we decided to fix $E_{\text {cut,low }}$ at $10 \mathrm{keV}$, which is approximately the lower bound for nonthermal electrons found in CSFs case studies (Fleishman et al. 2011, 2016; Motorina et al. in preparation).

For some events fitting by brmThick2PL model gave unstable solutions and high correlations between parameters. This means, that the data agree with a broad range of fitting parameters, and we should choose the most physically valid ones among them. Thus, in those cases we froze high energy spectral index according to thick-target model relationship between the photon and electron spectral indices $\delta_{2}=\gamma_{2}+1$ (Brown
1971; Somov \& Syrovatskii 1976). In some cases spectral steepening at energies $\geq 200 \mathrm{keV}$ was very sharp and number of counts in this region was not enough to determine $\delta_{2}$, then we determined just lower limits of $\delta_{2}$.

For all events fitted with the photon PL model, the brmThickPL model was used, and most events with the energy break in the photon spectrum also have a break in the electron spectrum, except 1 CSF, 2002-Aug-20, which was fitted by the 2PL model in the photon domain, while with a single brmThickPL model in the electron domain.

Distributions of the spectral parameter $\delta$ and the electron flux for brmThickPL model are presented in Table 5 and Figure 11(a-b). Likewise the photon spectral index, the electron spectral index is harder for CSFs, the median value is 4.1 , than for the reference flares, the median value equals to 4.3 , and this difference is rather significant: the probability that $\gamma$ distributions for CSFs and the reference group match is only $0.7 \%$. The electron flux for CSFs is weaker than for the reference set, the median values are $8.0 \times 10^{35} \mathrm{el} \mathrm{keV}^{-1} s^{-1}$ and $18.0 \times 10^{35} \mathrm{el} \mathrm{keV}^{-1} s^{-1}$, respectively. The electron flux ranges from $\sim 0.4$ to $\sim 300 \times 10^{35} \mathrm{el} \mathrm{keV}^{-1} s^{-1}$ for CSFs, and from $\sim 1$ to $\sim 300 \times 10^{35} \mathrm{el} \mathrm{keV}^{-1} s^{-1}$ for the reference group.

Distributions of the spectral parameters $\delta_{1}, E_{\text {break,el }}$, $\delta_{2}$, and the electron flux for the brmThick2PL model are presented in Table 5 and Figure 11(c-f). The median values of the electron low energy power-law index $\delta_{1}$ for CSFs and the reference group are 2.5 and 3.2, respectively, which is very close to the median values for $\gamma_{1}$ in Figure [10. A probable reason why $\delta_{1}$ and $\gamma_{1}$ strongly deviate from the expected relationship $\gamma_{1}=\delta_{1}-1$ for both groups can be a large contributions from higher energy electrons to the photon spectrum at lower energies. Distributions of the electron spectrum break energies $E_{\text {break,el }}$ are presented in Figure 11(d). The median values for two groups are close to each other: $188 \mathrm{keV}$ for CSFs, $158 \mathrm{keV}$ for the reference group. Probability of distribution equality is large; $\mathrm{p}=54 \%$. Distributions of the electron high-energy power-law index $\delta_{2}$ demonstrate that the reference flares might be slightly harder than CSFs, but this difference is insignificant, $\mathrm{p}=44 \%$. The median value of CSF electron flux is much smaller than that for the reference group: $1.3 \times 10^{35} \mathrm{el} \mathrm{keV}^{-1} \mathrm{~s}^{-1}$ vs. $7.2 \times 10^{35}$ el $\mathrm{keV}^{-1} \mathrm{~s}^{-1}$. Probability that those distributions for CSFs and the reference group are equal is low, $\mathrm{p}=0.3 \%$.

\subsection{Cold Solar Flare Timescales in HXR}

The duration of the impulsive flare phase in HXR was estimated using $t_{90}$ in G2 channel (see Section 3.1 and Figure 4). Values of $t_{90}$ for CSFs are listed in Table 3 and distributions of $t_{90}$ for the reference group and CSFs are presented in Figure 12. It is apparent that CSFs are significantly shorter than flares from the reference group: the median duration of CSFs is $8 \mathrm{~s}$, while for the reference set is $48 \mathrm{~s}$. Most CSFs have $t_{90}$ between 5 and $20 \mathrm{~s}, 5$ events have $t_{90}$ between $\sim 2$ and $\sim 5 \mathrm{~s}$, while there are 3 relatively long outliers: the longest event is 2000-May-18, $22: 59 \mathrm{UT},\left(t_{90} \simeq 86 \mathrm{~s}\right)$, and the other two are flare 2000$\operatorname{Mar}-18\left(t_{90} \simeq 23 \mathrm{~s}\right)$ and flare 2014-Oct-18 $\left(\mathrm{t}_{90} \simeq 63 \mathrm{~s}\right)$.

3.4. Spectral Properties of the Microwave Bursts 
TABLE 5

BRMPL AND BRM2PL FIT RESULTS.

\begin{tabular}{|c|c|c|c|c|c|c|c|c|}
\hline $\mathrm{N}$ & Date & $t_{0}{ }^{*}$, hh:mm:ss & $\delta_{1}$ & $\begin{array}{c}E_{\text {break,el }} \\
\text { keV }\end{array}$ & $\delta_{2}$ & $\begin{array}{c}\text { Electron flux } \\
10^{35} \mathrm{el} / \mathrm{s}\end{array}$ & $\chi^{2} /$ dof & Prob. \\
\hline 1 & 1998-Мay-07 & 05:32:37.072 & $2.7_{-0.5}^{+0.3}$ & $188_{-27}^{+37}$ & $5.3^{* *}$ & $0.8_{-0.5}^{+0.7}$ & $52.51 / 58$ & $6.8 \mathrm{e}-01$ \\
\hline 2 & 1999-Jun-19 & $22: 54: 49.788$ & $2.7_{-0.3}^{+0.5}$ & $242_{-21}^{+25}$ & $6.6^{* *}$ & $0.41_{-0.16}^{+0.22}$ & $48.00 / 58$ & $8.1 \mathrm{e}-01$ \\
\hline 3 & 1999-Jul-30 & 22:58:09.675 & $4.09_{-0.15}^{+0.16}$ & $\ldots$ & $\ldots$ & $6.5_{-1.9}^{+2.7}$ & $11.18 / 26$ & $9.9 \mathrm{e}-01$ \\
\hline 4 & 1999-Nov-09 & $08: 26: 21.703$ & $2.8_{-0.3}^{+0.2}$ & $213_{-44}^{+56}$ & $6.3_{-0.9}^{+3.3}$ & $2.3_{-1.0}^{+1.0}$ & $39.30 / 41$ & $5.4 \mathrm{e}-01$ \\
\hline 5 & 1999-Nov-14 & $14: 55: 08.244$ & $3.45_{-0.24}^{+0.24}$ & $\ldots$ & $\ldots$ & $0.39_{-0.22}^{+0.39}$ & $24.44 / 26$ & $5.5 \mathrm{e}-01$ \\
\hline 6 & 1999-Dec-02 & 20:01:00.012 & $4.29_{-0.15}^{+0.17}$ & $\ldots$ & $\ldots$ & $9.8_{-2.8}^{+4.1}$ & $23.41 / 25$ & $5.5 \mathrm{e}-01$ \\
\hline 7 & 2000-Mar-10 & $04: 21: 48.688$ & $4.12_{-0.13}^{+0.14}$ & $\ldots$ & $\ldots$ & $8.0_{-2.1}^{+2.8}$ & $27.83 / 26$ & $3.7 \mathrm{e}-01$ \\
\hline 8 & 2000-Mar-18 & $02: 25: 10.567$ & $3.2_{-0.4}^{+0.3}$ & $124_{-10}^{+12}$ & $6.2^{* *}$ & $2.0_{1.0}^{+1.3}$ & $43.40 / 58$ & $9.2 \mathrm{e}-01$ \\
\hline 9 & 2000-May-18 & 07:21:59.706 & $3.0_{-0.3}^{+0.3}$ & $145_{-11}^{+12}$ & $7.1^{* *}$ & $1.3_{-0.5}^{+0.7}$ & $67.70 / 59$ & $2.0 \mathrm{e}-01$ \\
\hline 10 & 2000-May-18 & $22: 59: 39.777$ & $4.59_{-0.11}^{+0.12}$ & $\ldots$ & $\ldots$ & $35_{-7}^{+9}$ & $29.73 / 25$ & $2.3 \mathrm{e}-01$ \\
\hline 11 & 2001-Oct-12 & 07:40:31.941 & $3.96_{-0.10}^{+0.10}$ & $\cdots$ & $\cdots$ & $8.0_{-1.7}^{+2.2}$ & $35.23 / 27$ & $1.3 \mathrm{e}-01$ \\
\hline 12 & 2001-Nov-01 & $15: 17: 42.772$ & & $\ldots$ & $\cdots$ & -1.1 & $\ldots$ & $\ldots$ \\
\hline 13 & 2002-Мay-29 & $07: 39: 46.864$ & $\begin{array}{l}4.12_{-0.09}^{+0.09}\end{array}$ & $\ldots$ & $\cdots$ & $15.3_{-2.8}^{+3.4}$ & $29.91 / 25$ & $2.3 \mathrm{e}-01$ \\
\hline 14 & 2002-Aug-10 & 23:50:09.293 & $\ldots$ & $\cdots$ & $\cdots$ & $\ldots$ & $\ldots$ & $\ldots$ \\
\hline 15 & 2002-Aug-18 & $23: 11: 19.740$ & $4.02_{-0.16}^{+0.18}$ & $\cdots$ & $\cdots$ & $5.2_{-1.7}^{+2.5}$ & $31.78 / 26$ & $2.0 \mathrm{e}-01$ \\
\hline 16 & 2002-Aug-20 & $19: 55: 28.476$ & $3.77_{-0.08}^{+0.08}$ & $\ldots$ & $\ldots$ & $8.1_{-1.5}^{+1.9}$ & $45.06 / 33$ & $7.9 \mathrm{e}-02$ \\
\hline 17 & 2003-Oct-23 & $22: 17: 39.620$ & $2.5_{-0.5}^{+0.4}$ & $140_{-16}^{+22}$ & $6.8_{-0.7}^{+1.6}$ & $2.1_{-1.0}^{+1.0}$ & $46.00 / 44$ & $3.9 \mathrm{e}-01$ \\
\hline 18 & 2005-Sep-08 & 02:15:49.996 & $2.0_{-0.4}^{+0.3}$ & $390_{-45}^{+53}$ & $5.8^{* *}$ & $0.31_{-0.15}^{+0.23}$ & $71.10 / 54$ & $5.9 \mathrm{e}-02$ \\
\hline 19 & 2011-Sep-19 & $07: 43: 40.791$ & $4.03_{-0.13}^{+0.14}$ & $\ldots$ & $\cdots$ & $6.6_{-1.8}^{+2.4}$ & $18.16 / 23$ & $7.5 \mathrm{e}-01$ \\
\hline 20 & 2012-Jul-08 & $02: 43: 50.647$ & $4.15_{-0.19}^{+0.22}$ & $\ldots$ & $\ldots$ & $10_{-4}^{+6^{\circ}}$ & $47.00 / 25$ & $5.0 \mathrm{e}-03$ \\
\hline 21 & 2013-Nov-05 & $03: 50: 24.588$ & $3.50_{-0.11}^{+0.12}$ & $\ldots$ & $\ldots$ & $1.5_{-0.4}^{+0.6}$ & $26.50 / 24$ & $3.3 \mathrm{e}-01$ \\
\hline 22 & 2014-Jan-02 & 05:45:01.390 & $4.03_{-0.15}^{+0.17}$ & $\cdots$ & $\ldots$ & $4.1_{-1.3}^{+1.8}$ & $32.33 / 22$ & $7.2 \mathrm{e}-02$ \\
\hline 23 & 2014-Jan-31 & $16: 52: 37.461$ & $4.98_{-0.11}^{+0.11}$ & $\ldots$ & $\ldots$ & $95_{-17}^{+21}$ & $43.80 / 21$ & $3.0 \mathrm{e}-03$ \\
\hline 24 & 2014-Feb-08 & $05: 49: 29.848$ & $4.16_{-0.12}^{+0.13}$ & $\cdots$ & $\cdots$ & $9.4_{-2.2}^{+2.9}$ & $36.42 / 24$ & $5.0 \mathrm{e}-02$ \\
\hline 25 & 2014-Oct-18 & 02:49:17.710 & & $\cdots$ & $\cdots$ & & $\ldots$ & $\cdots$ \\
\hline 26 & 2014-Oct-27 & $03: 14: 46.862$ & $4.15_{-0.21}^{+0.23}$ & $\cdots$ & $\cdots$ & $5.6_{-2.2}^{+3.6}$ & $16.91 / 22$ & $7.7 \mathrm{e}-01$ \\
\hline 27 & 2015-May-07 & $12: 41: 40.415$ & $3.61_{-0.13}^{+0.14}$ & $\cdots$ & $\cdots$ & $1.8_{-0.6}^{+0.8}$ & $29.83 / 27$ & $3.2 \mathrm{e}-01$ \\
\hline
\end{tabular}

${ }^{*}$ The Konus- Wind trigger time after corrections for the light propagation to the Earth are applied. ** Lower limits.

Given that the data files (IDL sav files) were created in a format identical to the OVSA data files, we took advantage of having the OVSA software from the SSW library. Specifically, the composite OVSA-like sav files have been read by OVSA_Explorer widget, which has all functionality for data handling including the background subtraction and sequential spectral fitting.

Here we are only interested in the microwave bursts produced by gyrosynchrotron (GS) emission of energetic electrons, but ignore any component that could be attributed to a coherent plasma emission. GS spectrum $S(f)$ is characterized by a maximum flux density $S_{\text {peak }}$ at a frequency $f_{\text {peak }}$ and two spectral slopes $\alpha_{\text {lf }}$ in the low frequency range and $\alpha_{\mathrm{hf}}$ in the high frequency range (Stahli et al. 1989). We performed spectral fitting with the OVSA_Explorer built-in generic spectral function:

$$
S=e^{A} f^{\alpha}\left[1-e^{-e^{B} f^{-\beta}}\right]
$$

where $f$ is the frequency in $\mathrm{GHz}$, while $A, B, \alpha$, and $\beta$ are the free fitting parameters, which yield the physical parameters of interest. For example, the low-frequency spectral index $\alpha_{\text {lf }} \equiv \alpha$, while the high-frequency spectral index is $\alpha_{\mathrm{hf}}=\alpha-\beta$. The peak frequency $f_{\text {peak }}$ and the flux density at the peak frequency $S_{\text {peak }}$ are calculated via parameters of the function $S$ (see Nita et al. 2004, for more detail).
Since microwave data for different events have dissimilar time cadences, for spectral parameter analysis we first brought all the data to the same cadence selected to be $1 \mathrm{~s}$. To this end the multifrequency time profiles of three OVSA events available with the cadence of 5 or 4 seconds were interpolated to 1 second resolution using IDL interpolate routine; in the remaining cases the available 1 s data were used, which implies that in case of NoRP data we used the $1 \mathrm{~s}$ background data rather than the flare mode data. However, when the high-frequency light curves were deemed critical for the fitting, we also added the time-averaged NoRP $80 \mathrm{GHz}$ light curve available in the flare mode only to the background $1 \mathrm{~s}$ record.

Having the combinations of the microwave data recorded by different instruments raises several problems with the data handling and analysis. Three main problems are (i) different flux calibrations at different instruments, (ii) different background levels, and (iii) possible clock errors and, thus, time scale mismatches. We dealt with all these issues individually for each event. As it was mentioned above, we used the NoRP clock as the reference time and adjusted clocks of other instruments using the lag-correlation between the light curves (cf., Fleishman et al. 2016). Total flux corrections were introduced based on comparison of the preflare signal levels, but this correction was not always successful; see prominent horizontal stripes in a few panels of Figure 13. most 

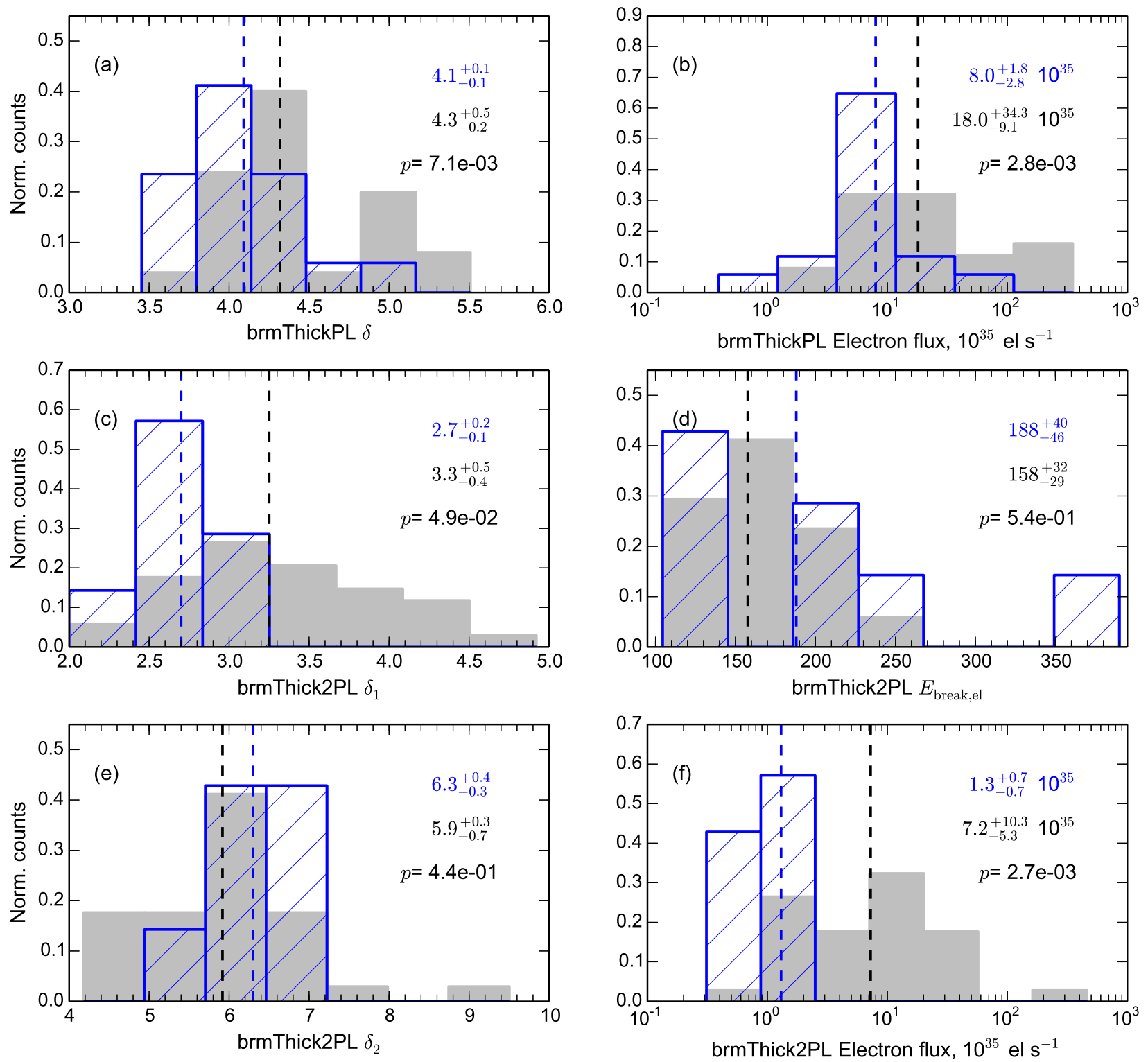

FIG. 11. - Parameter distributions for electron spectra obtained from thick-target model. Bin heights are normalized to total number of events in each group. Blue hatched histograms refer to cold flares, grey histograms - to reference group. Median values and 0.5 probability ranges are presented on each plot for cold flares and for reference group in blue and black letters respectively. Black "p" indicates twosided p-value from Kolmogorov-Smirnov test in suggestion that distributions of a given parameter for cold flares and reference flares are equal. (a) brmThickPL electron spectral index, $\delta$, distributions; (b) brmThickPL electron flux distributions in $10 \mathrm{keV}-40 \mathrm{MeV}$ range; (c) brmThick2PL electron spectral index in lower energy range, $\delta_{1}$, distributions; (d) brmThick2PL break energy of electron spectrum, $\mathrm{E}_{\text {break,el }}$, distributions; (e) brmThick2PL photon spectral index in higher energy range, $\delta_{2}$, distributions; (f) brmThick2PL electron flux distributions in $10 \mathrm{keV}-40000 \mathrm{MeV}$ range.

notably, the upper left panel.

The frequency- and time- dependent background level was subtracted manually using the corresponding builtin functionality of the OVSA_Explorer, which allows defining a flat or polynomial background for each frequency channel. In most cases it was sufficient to subtract a flat off-burst background level, although in some cases the background was time-dependent. In such cases the background was approximated by an appropriate polynomial. In one case (2003-Oct-23), observed with both NoRP and OVSA, the short radio burst of interest occurred on top of a much more gradual broadband burst, whose dynamic spectrum is shown in the upper left panel of Figure 14. The (sub)burst of interest is seen at this dynamic spectrum as a short red dash at around 22:17:40 UT at the high-frequency end of the spectrum. One can notice a few more similar bright short subbursts later in the event; they are not artifacts as the same subbursts are detected by NoRP with higher time resolution; see the corresponding panel in Figure 13. Thus, for our quantitative analysis of this event we use the $1 \mathrm{~s}$ cadence NoRP +RSTN data from which the gradual burst emis- 
TABLE 6

MICROWAVE PROPERTIES OF COLD SOLAR FLARES.

\begin{tabular}{|c|c|c|c|c|c|c|c|c|c|c|}
\hline $\mathrm{N}$ & Date & $\begin{array}{c}t_{0}{ }^{*} \\
\text { hh:mm:ss }\end{array}$ & MW instr. & $\begin{array}{c}\Delta t \\
\mathrm{~s}\end{array}$ & $\begin{array}{c}\left\langle S_{\text {peak }}\right\rangle \\
\text { SFU }\end{array}$ & $\begin{array}{c}\left\langle f_{\text {peak }}\right\rangle \\
\mathrm{GHz}\end{array}$ & $\left\langle\alpha_{\mathrm{lf}}\right\rangle$ & $\left\langle\alpha_{\mathrm{hf}}\right\rangle$ & 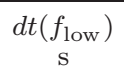 & $d t\left(\underset{\mathrm{s}}{\left.f_{\text {high }}\right)}\right.$ \\
\hline 1 & 1998-Мау-07 & $05: 32: 37.072$ & NoRP+RSTN & 16 & $30 \pm 10$ & $1.3 \pm 0.8$ & $1.9 \pm 0.5$ & $-1.3 \pm 0.6$ & $2.0 \pm 1.0$ & $1.5 \pm 1.0$ \\
\hline 2 & 1999-Jun-19 & $22: 54: 49.788$ & NoRP & $\ldots$ & $\ldots$ & $\ldots$ & $\ldots$ & $\ldots$ & $\ldots$ & $\ldots$ \\
\hline 3 & 1999-Jul-30 & $22: 58: 09.675$ & NoRP & $\ldots$ & $40^{* *}$ & $17^{* *}$ & $\ldots$ & $\ldots$ & $1.5 \pm 1.0$ & $1.5 \pm 1.0$ \\
\hline 4 & 1999-Nov-09 & $08: 26: 21.703$ & RSTN & 28 & $35 \pm 14$ & $4.7 \pm 0.6$ & $0.5 \pm 0.3$ & $-2.7 \pm 0.7$ & & \\
\hline 5 & 1999-Nov-14 & $14: 55: 08.244$ & RSTN & $\ldots$ & & $\ldots$ & $\ldots$ & $\ldots$ & $-4.5 \pm 1.0$ & $-5.6 \pm 1.0$ \\
\hline 6 & 1999-Dec-02 & 20:01:00.012 & RSTN & 14 & $2.9 \pm 0.5$ & $12 \pm 3$ & $0.29 \pm 0.12$ & $-3 \pm 2 \mathrm{v}$ & $2.1 \pm 1.0$ & $2.4 \pm 1.0$ \\
\hline 7 & 2000-Mar-10 & $04: 21: 48.688$ & NoRP+RSTN & $\ldots$ & $110^{* *}$ & $35^{* *}$ & $\ldots$ & $\ldots$ & $1.3 \pm 1.0$ & $1.5 \pm 1.0$ \\
\hline 8 & 2000-Mar-18 & $02: 25: 10.567$ & NoRP+RSTN & 53 & $166 \pm 72$ & $6.3 \pm 1.7$ & $3.1 \pm 0.9$ & $-3.4 \pm 1.6$ & $0.0 \pm 1.0$ & $-1.8 \pm 1.0$ \\
\hline 9 & 2000-May-18 & $07: 21: 59.706$ & NoRP & $\ldots$ & & & & & $4.9 \pm 1.0$ & $110.8 \pm 1.0$ \\
\hline 10 & 2000-May-18 & $22: 59: 39.777$ & NoRP, OVSA & 67 & $67 \pm 24$ & $13 \pm 0.8$ & $1.19 \pm 0.29$ & $-5.1 \pm 1.7$ & $17.4 \pm 1.0$ & $12.7 \pm 1.0$ \\
\hline 11 & 2001-Oct-12 & $07: 40: 31.941$ & (1) & $\cdots$ & & & & & & \\
\hline 12 & 2001-Nov-01 & $15: 17: 42.772$ & RSTN & 11 & $52 \pm 20$ & $1.43 \pm 0.05$ & $2.6 \pm 0.8$ & $-1.7 \pm 0.6$ & $5.0 \pm 1.0$ & $2.7 \pm 1.0$ \\
\hline 13 & 2002-Маy-29 & $07: 39: 46.864$ & NoRP+RSTN & 7 & $36 \pm 19$ & $14.4 \pm 12.0$ & $1.4 \pm 1.0$ & $-2.5 \pm 1.7$ & $1.1 \pm 1.0$ & $1.0 \pm 1.0$ \\
\hline 14 & 2002-Aug-10 & 23:50:09.293 & OVSA & $\ldots$ & $5^{* *}$ & $18^{* *}$ & $\ldots$ & $\ldots$ & $\ldots$ & $\ldots$ \\
\hline 15 & 2002-Aug-18 & $23: 11: 19.740$ & $\begin{array}{c}\text { NoRP }+ \text { RSTN } \\
\text { OVSA }\end{array}$ & 34 & $15 \pm 7$ & $9.4 \pm 2.5$ & $1.3 \pm 0.3$ & $-2.6 \pm 1.5$ & $3.6 \pm 1.0$ & $0.1 \pm 1.0$ \\
\hline 16 & 2002-Aug-20 & $19: 55: 28.476$ & RSTN & $\cdots$ & $110^{* *}$ & $15.4^{* *}$ & $\ldots$ & & & \\
\hline 17 & 2003-Oct-23 & $22: 17: 39.620$ & $\begin{array}{c}\text { NoRP+RSTN } \\
\text { OVSA }\end{array}$ & 13 & $282 \pm 176$ & $20 \pm 3$ & $3.2 \pm 0.6$ & $-3.2 \pm 2.3$ & $74.8 \pm 1.0$ & $2.1 \pm 1.0$ \\
\hline 18 & 2005-Sep-08 & $02: 15: 49.996$ & NoRP & $\cdots$ & $\cdots$ & & $\ldots$ & 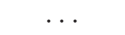 & & \\
\hline 19 & 2011-Sep-19 & $07: 43: 40.791$ & NoRP+RSTN & 16 & $23 \pm 11$ & $7 \pm 6$ & $3.5 \pm 1.7$ & $-0.9 \pm 0.5$ & $0.0 \pm 1.0$ & $1.8 \pm 1.0$ \\
\hline 20 & 2012-Jul-08 & $02: 43: 50.647$ & $\begin{array}{l}\text { NoRP+SRS } \\
\text { +RSTN }\end{array}$ & 8 & $60 \pm 22$ & $12.1 \pm 1.8$ & $2.4 \pm 0.7$ & $-1.3 \pm 0.7$ & $1.6 \pm 1.0$ & $0.2 \pm 1.0$ \\
\hline 21 & 2013-Nov-05 & $03: 50: 24.588$ & $\begin{array}{l}\text { NoRP+SRS } \\
+ \text { RSTN }\end{array}$ & 11 & $202 \pm 71$ & $23.1 \pm 2.3$ & $1.96 \pm 0.27$ & $-3.0 \pm 0.7$ & $5.4 \pm 1.0$ & $1.3 \pm 1.0$ \\
\hline 22 & 2014-Jan-02 & 05:45:01.390 & NoRP+SRS & 9 & $22 \pm 7$ & $12.7 \pm 0.6$ & $1.8 \pm 0.8$ & $-2.6 \pm 1.4$ & $5.8 \pm 1.0$ & $3.1 \pm 1.0$ \\
\hline 23 & 2014-Jan-31 & $16: 52: 37.461$ & RSTN & $\ldots$ & $54^{* *}$ & $15.4^{* *}$ & $\ldots$ & $\ldots$ & $7.7 \pm 1.0$ & $6.5 \pm 1.0$ \\
\hline 24 & 2014-Feb-08 & $05: 49: 29.848$ & NoRP & $\ldots$ & $55^{* *}$ & $17^{* *}$ & $\ldots$ & $\ldots$ & $-0.4 \pm 1.0$ & $0.1 \pm 1.0$ \\
\hline 25 & 2014-Oct-18 & $02: 49: 17.710$ & NoRP+BBMS & 195 & $69 \pm 37$ & $8.1 \pm 0.4$ & $3.3 \pm 0.5$ & $-1.3 \pm 0.3$ & $0.8 \pm 1.0$ & $0.5 \pm 1.0$ \\
\hline 26 & 2014-Oct-27 & $03: 14: 46.862$ & NoRP+SRS & 16 & $175 \pm 95$ & $13.3 \pm 2.4$ & $4.9 \pm 2.9$ & $-1.2 \pm 0.5$ & $6.0 \pm 1.0$ & $3.4 \pm 1.0$ \\
\hline 27 & 2015-May-07 & $12: 41: 40.415$ & RSTN+KMAS & $\cdots$ & $45^{* *}$ & $15.4^{* *}$ & $\ldots$ & $\ldots$ & $1.6 \pm 1.0$ & $3.2 \pm 1.0$ \\
\hline
\end{tabular}

* The Konus- Wind trigger time after corrections for the light propagation to the Earth are applied.

** Lower limits.

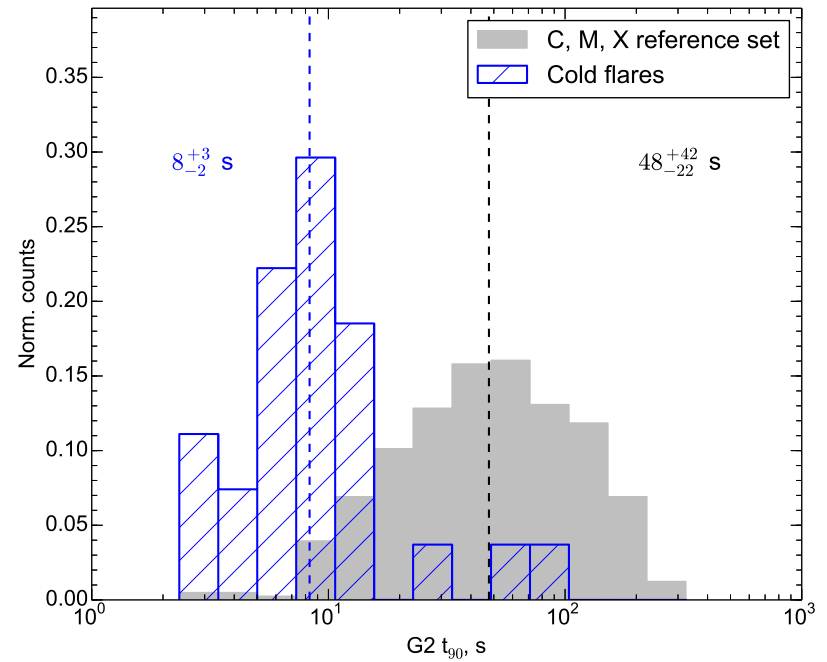

FIG. 12.- HXR duration estimation, $t_{90}$, distribution defined on G2 channel. Bin heights are normalized to total number of events in each group. Blue shaded bins refer to cold flares, grey bins refer to reference set of C-, M- and X-class flares. Dashed lines indicate median values for cold flares (blue) and reference set (black), labels refer to median values and 0.5 range for cold events (blue) and reference set (black). sion was subtracted5. As a result, a data set with the background subtracted has been created and separately saved; the microwave dynamic spectra for all these events are given in Figures 13 and 14.

This set of the background-subtracted dynamic spectra was used to perform sequential spectral fit for each $1 \mathrm{~s}$ time frame. The spectral fitting frequency range was chosen individually for each event to contain the main microwave component; we excluded low-frequency channels if a secondary, presumably coherent, component was present there, or high-frequency channels in case they were too noisy to aid the fitting. Although every attempt has been made to create accurate data files, the successful spectral fit was possible for only slightly more than half of all events (15 of 26); the corresponding dynamic spectra are gathered in Figure 13. In the remaining 11 cases the fit failed entirely 6 ; see Figure [14. Visual inspection of Figure 14 suggests that fit might fail for the following reasons: (i) too small number of channels with a meaningful signal and (ii) too high spectral peak frequency $(15.4-35 \mathrm{GHz})$ such as a good fraction of the high-frequency microwave spectrum is outside the spec-

5 This bursts shares some apparent properties with the 2002Mar-10 cold flare with delayed heating (Fleishman et al.|2016) but studying the delayed component is beyond the scope of this statistical study.

6 The fit is possible for the left upper case, but this is the case treated as a background for a more impulsive subburst as explained above. 


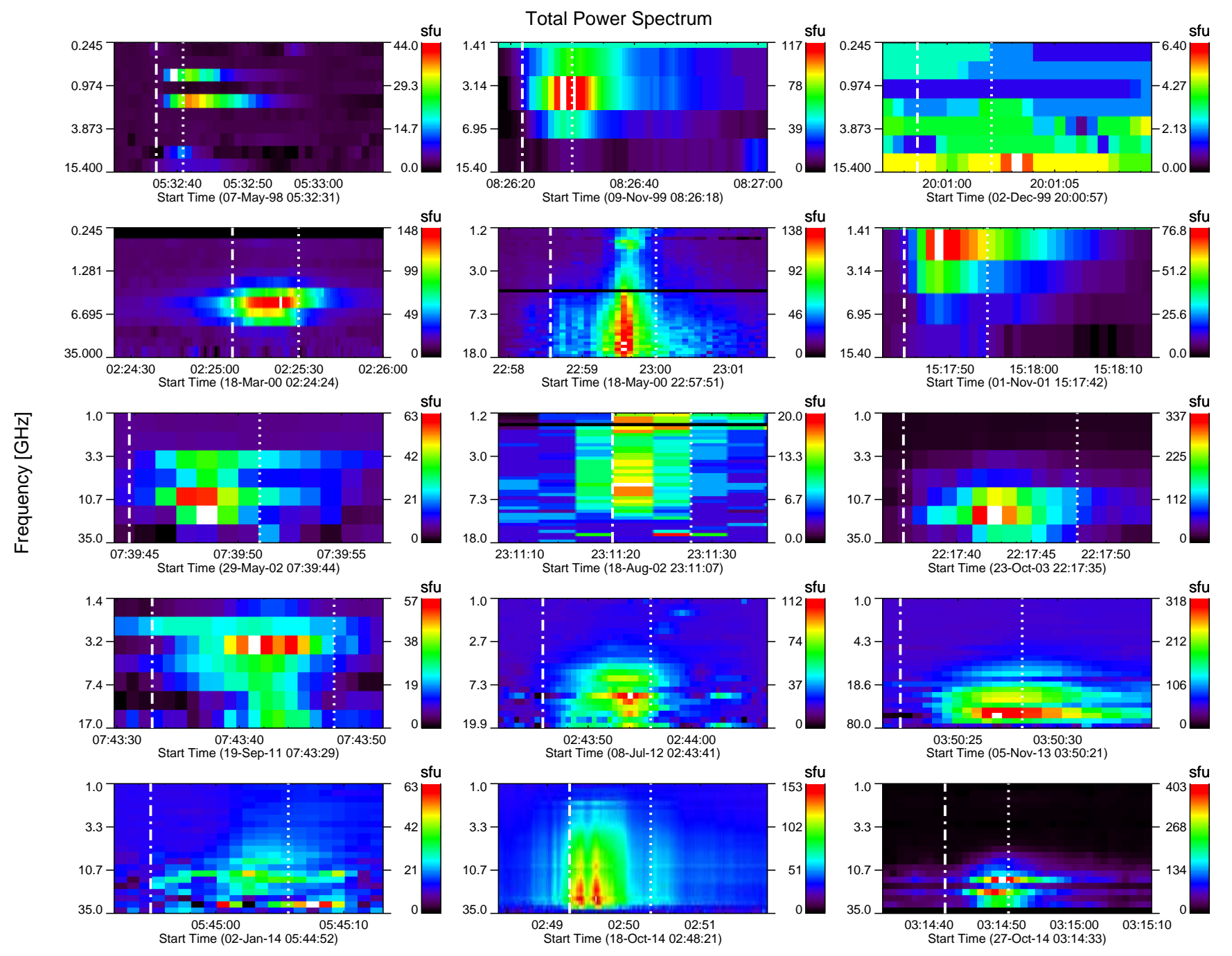

FIG. 13.- Microwave dynamic spectra for 15 flares with successful spectral fits. Vertical dash-dotted line corresponds to the flare beginning in HXR range $t_{5}$, vertical dotted line corresponds to the flare ending in HXR range $t_{95}$ (see Section 3.1 ).

tral coverage of the available instruments, which is the case for 5 of 11 events with no fit. The fit results for every successful time frame were saved in specifically designed OVSA med files, which are in fact a special form of the IDL sav files (see Nita et al. 2004, for more detail)7.

It should be noted that even though the data were processed to create a database as uniform as possible, there are unavoidably some biases related to the individual instrument limitations and some anomalous features of the bursts. We will return to these biases later, when discussing the implications and significance of the results of our statistical analysis.

As a reference group in the microwave domain we used the database from Nita et al. (2004) kindly provided by Dr. Gelu M. Nita. The microwave emission in our sample of events is undoubtedly the incoherent gyrosynchotron emission even though the spectral peak frequencies vary in a wide range between $\sim 1 \mathrm{GHz}$ and $35 \mathrm{GHz}$. The reference group of events, reported

7 The created database of the composite microwave spectra is available at http://www.ioffe.ru/LEA/SF_AR/Radio.html by Nita et al. (2004), contains both gyrosynchotron and coherent bursts. Nita et al. (2004) found an empirical boundary at $2.6 \mathrm{GHz}$, which demarcates decimetric (Dtype; often-coherent) and centimetric (C-type; mainlygyrosynchotron) bursts. In addition, a group of bursts with multiple spectral peaks in both centimeter and decimeter ranges was separated and called CD-type. For a fair comparison between our event sample and the reference set, we only included the C-type bursts and the centimeter component of CD-type bursts in the statistical distribution of the reference group. This resulted in minor deviations in our numbers compared with those presented by Nita et al. (2004), but those deviations are not statistically significant.

As microwave spectral parameters may vary significantly during the microwave burst duration (Melnikov et al. 2008; Fleishman et al. 2016) for the statistical study we used parameters obtained on each time frame with a successful fit during each microwave burst peak. Peak durations were estimated as time intervals during which the flux density at the local peak frequency is above $80 \%$ of the corresponding 

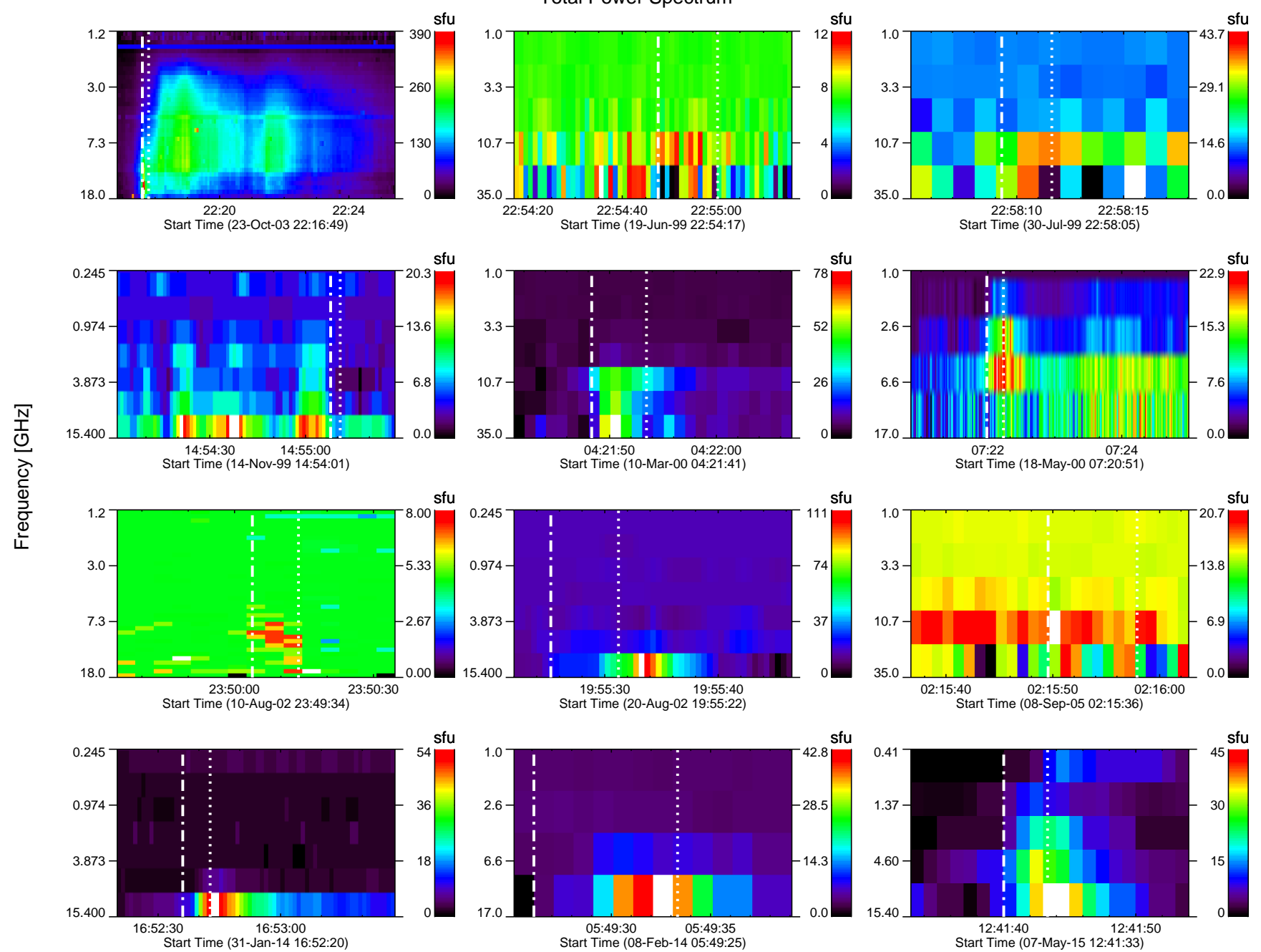

FIG. 14.- Microwave dynamic spectra for 11 flares for which spectral fitting didn't succeed and long duration spectrum for 23-Oct-03, which was not used in microwave spectral parameter distributions. Vertical dash-dotted line corresponds to the flare beginning in HXR range $t_{5}$, vertical dotted line corresponds to the flare ending in HXR range $t_{95}$ (see Section 3.1).

peak flux (see Nita et al. 2004, for more detail).

Burst averaged microwave spectral parameters for cold solar flares are presented in Table [6] while comparison of the spectral parameters of CSFs with the reference set for each time frame is illustrated in Figure 15. Even though we used essentially the same set of data, the displayed parameter distributions for the reference group differ from those described by Nita et al. (2004) because we display slightly different set of entries - those, which are required for our study.

The peak frequency distributions for the CSFs and the reference set are presented in Figure 15, top left. Histogram bins are the same as in Nita et al. (2004) for more straightforward comparison of the results. For the peak frequency distribution of CSFs, a small maximum near $\sim 1.5 \mathrm{GHz}$ is observed followed by a broad minimum, then, after $2.6 \mathrm{GHz}$, the distribution function begins to grow and has two more maxima, one near $\sim 6^{-}$ $9 \mathrm{GHz}$, which is close to the distribution maximum for the reference group, and the second maximum between 11 and $18 \mathrm{GHz}$. Given that the histograms include the outcome of each time frame with a successful spectral fit, inputs from longer events have proportionally larger weights than those from shorter events. We checked and found that the two prominent peaks correspond to contributions from two long events: 2014-Oct-18 (the spectral peak frequency varies within $7-9 \mathrm{GHz}$ ) and 2000May-18, 22:59 UT (the spectral peak frequency varies within 11-18 GHz); we will return to this bias later. The median spectral peak frequency for CSFs is $11.2 \mathrm{GHz}$, which is significantly larger than the median value for the reference group, $6.2 \mathrm{GHz}$. In fact, the true mismatch between the spectral peak frequencies is even stronger. Indeed, we have already noticed from Figure 14 that there are many (five, which is $\sim 20 \%$ of the total number of CSFs) events, whose spectral peak frequency is outside the available spectral range, i.e., at least above 15.4 GHz; such values, if properly added to the distribution, would further increase the median value of the spectral peak frequency of CSFs. However, those events do not contribute to the histograms because no fit was possible for them. Although we used NoRP data with 

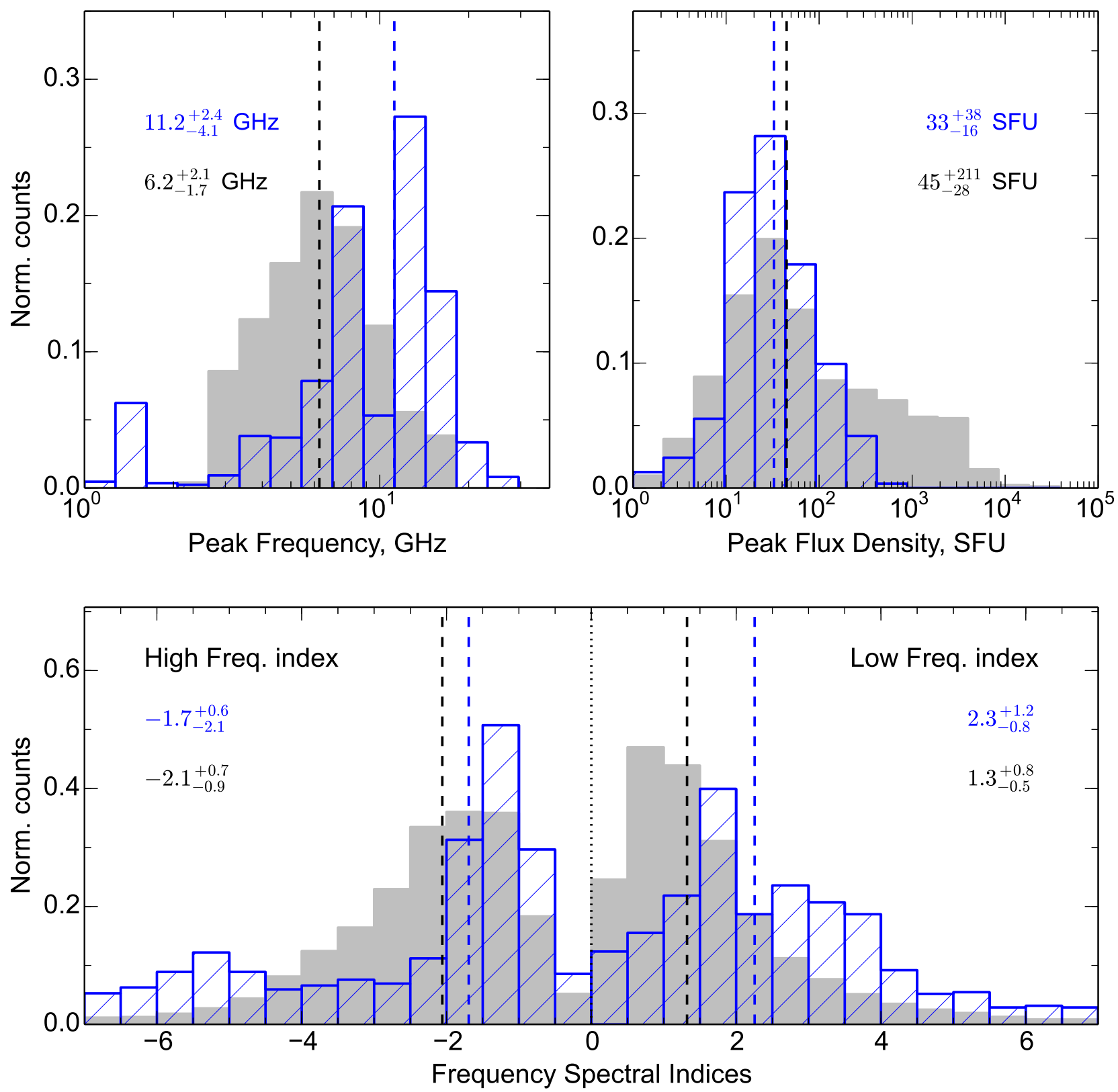

FIG. 15. - Microwave distributions of spectral parameters obtained on each time interval during each peak of microwave burst. Bin heights are normalized to total number of entries in each group. Top left: peak frequency distribution for cold solar flare s (shaded blue) and reference group (grey); top right: peak flux density distribution for cold solar flare s (shaded blue) and reference group (grey); bottom: spectral indices distributions for cold solar flare s (shaded blue) and reference group (grey). Median values and 0.5 probability ranges are presented on each plot.

wider frequency range extending up to $35 \mathrm{GHz}$ in the background mode, the region 11-18 GHz lies within the OVSA coverage used by Nita et al. (2004), thus we can conclude that the higher spectral peak frequencies in the CSF set are not due to a selection effect, but have a physical origin. It should, however, be noted that along with events having unusually high spectral peak frequencies, there are a few events with rather low peak frequencies, $f_{\text {peak }} \sim 2-3 \mathrm{GHz}$, similar to the cold, tenuous flare described by Fleishman et al. (2011).

Distributions of the peak flux density are plotted in Figure 15] top right. These distributions have bell shapes with maxima between 20 and $50 \mathrm{sfu}$. The median values for the CSFs and the reference flares are close to each other, being equal to $33 \mathrm{sfu}$ and $45 \mathrm{sfu}$, respectively. A minimum peak flux density for the time intervals where the spectral fitting for CSFs was successful is $\sim 4 \mathrm{sfu}$, while the maximum peak flux density is $\sim 600 \mathrm{sfu}$; the $50 \%$ probability range is from $17 \mathrm{sfu}$ to $71 \mathrm{sfu}$. The 

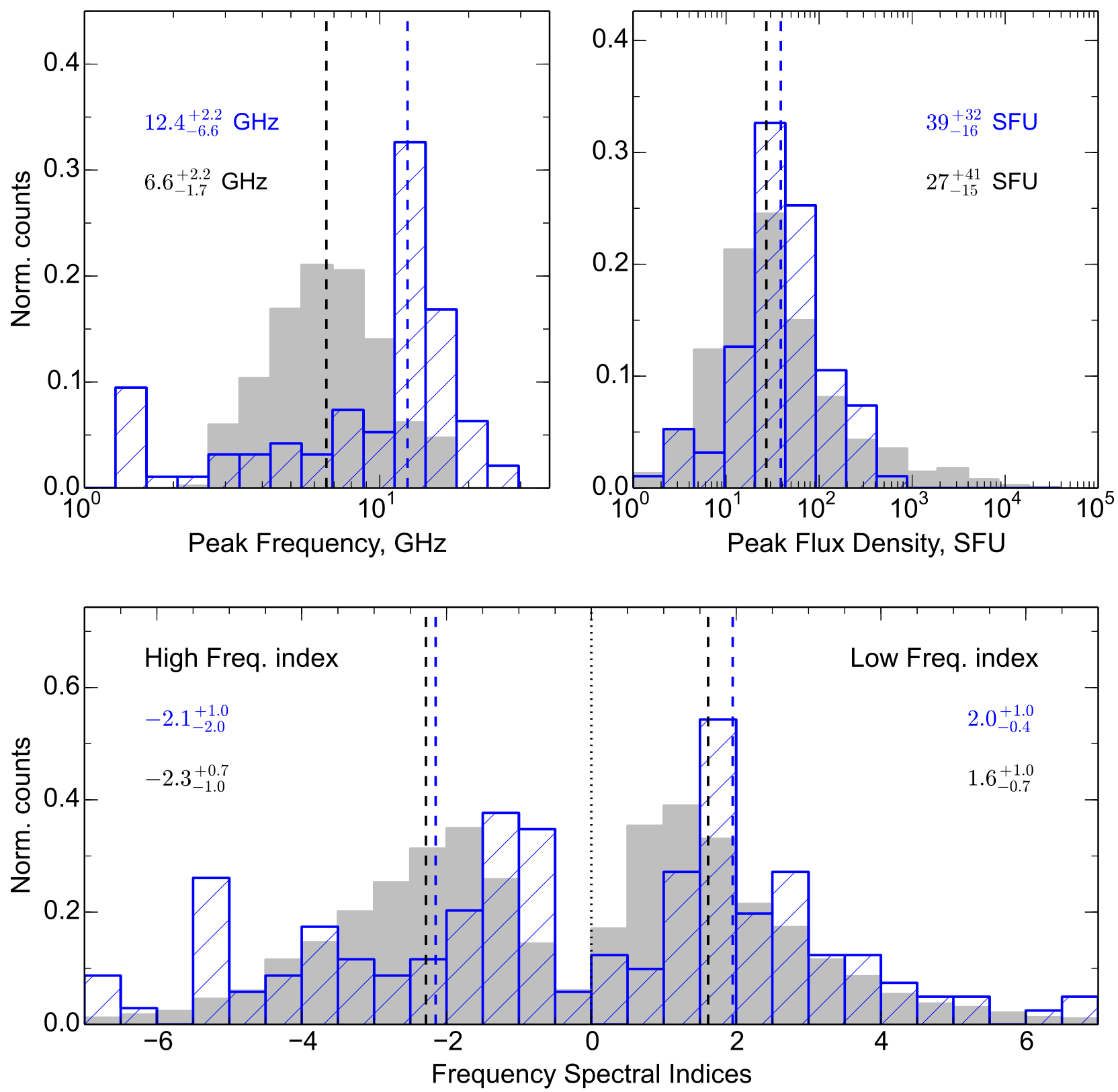

FIG. 16. - Microwave distributions of spectral parameters obtained on 5 time intervals during each peak of microwave burst. Bin heights are normalized to total number of entries in each group. Top left: peak frequency distribution for cold solar flare s (shaded blue) and reference group (grey); top right: peak flux density distribution for cold solar flare s (shaded blue) and reference group (grey); bottom: spectral indices distributions for cold solar flare s (shaded blue) and reference group (grey). Median values and 0.5 probability ranges are presented on each plot.

reference group $50 \%$ probability range is between $17 \mathrm{sfu}$ and $256 \mathrm{sfu}$; no extremely intense burst with a peak flux density >1000 sfu was found among the CSFs.

Distributions of high- and low- frequency spectral indices for both groups are presented in Figure 15, bottom. For the high-frequency spectral index (negative region) the CSF median value is -1.7 and $50 \%$ probability range is within -3.8 and -1.1 . The median value for the reference group is -2.1 ; thus, the CSFs are slightly harder, while $50 \%$ range for the reference flares is narrower and falls between -3.0 and -1.4 . The low frequency spectral index (positive region) median value for the CSFs is 2.3 , which is significantly larger than that for the reference group characterized by the median value of 1.3 , although, like in the case of high-frequency index distribution, the low-frequency slope distribution for CSFs has a wider $50 \%$ range, 1.5-3.5, than the reference set distribution, 0.8-2.1. Such wide ranges of the spectral indices for CSFs come from long distribution "tails" in the regions, where the spectral indices are large in absolute 


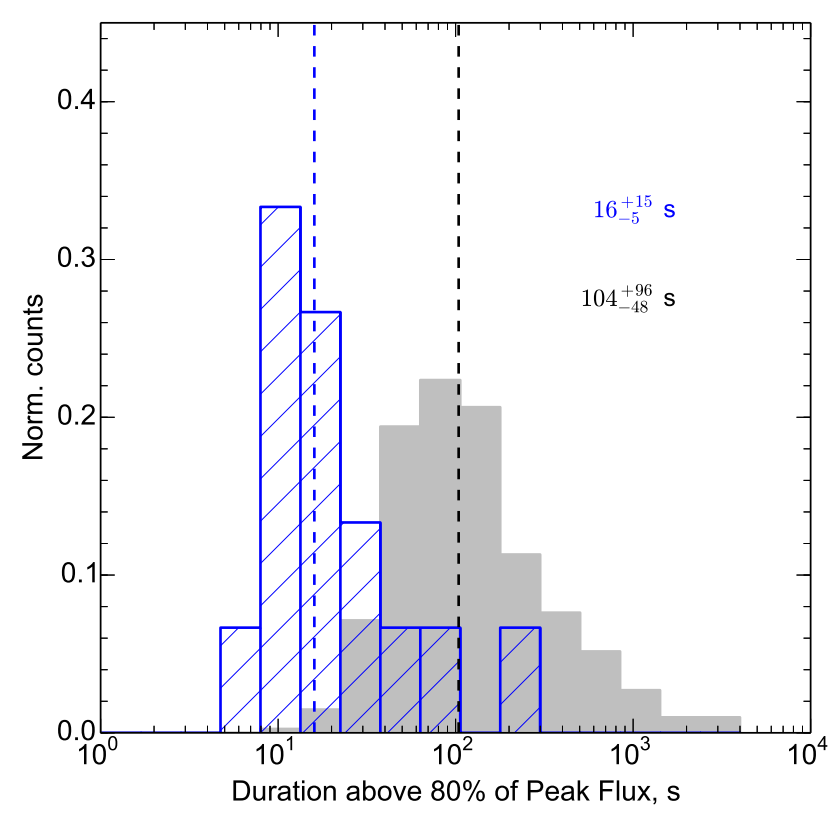

FIG. 17.- Microwave duration distribution for cold solar flares (shaded blue) and reference flares (grey). Bin heights are normalized to total number of events in each group. Median values and 0.5 probability ranges are presented on each plot.

value. Some contribution to these tails can come from fitting artifacts, where the low- or high- frequency slope is only constrained by one spectral channel in cases when the spectral peak frequency is either very large or small. In such cases the corresponding spectral index is determined with a large error. In what follows we do not draw any physical conclusion based on the presence of these tails. If we neglect the second peak (tail), i. e. values $\leq-4.5$, of the high-frequency spectral index histogram, the median value for reference flares doesn't change sigficantly and becomes -2.0 , while median for CSFs moves to -1.4 and coincides with the strongly pronounced maximum of CSF high frequency slope distribution.

The use of the entire flare duration in the statistics described above is justified by the fact that there are cases with an extremely prominent spectral evolution, such as in a cold flare described by Fleishman et al. (2016), where the spectral peak frequency varied within 1.5 orders of magnitude. In such cases, characterization of the event with only the peak value or a few 'representative' time frames could be misleading. On the other hand, a 'cold' flare reported by Fleishman et al. (2011) did not show any spectral evolution, so a single time frame would be sufficient to fully describe its spectral shape. To balance such extremes in our statistical study, we complement the described treatment relying on all successfully fitted time frames, and so giving an enhanced weight to longer bursts, by a treatment that characterizes each distinct temporal peak by exactly five time frames independently on the flare duration; thus, giving equal weight to every peak. These five time frames are selected at the beginning and at the end of the peak, at the peak maximum and in the middles of the raising and declining phases. The microwave spectral parameter distributions for the input specified this way are presented in Figure 16.
Compared to Figure [15, the most significant change is observed in the peak frequency distribution of CSFs; Figure 16. top left: the mid-frequency peak at $\sim 7 \mathrm{GHz}$ has almost gone, while the high-frequency one has raised such as the median value for CSFs is now $12.4 \mathrm{GHz}$, which is significantly larger than the median value for the reference group, 6.6 GHz.

Distributions of the peak flux density, Figure [16, top right, have changed only slightly. The median values for CSFs and reference flares are close to each other and are $39 \mathrm{SFU}$ and $27 \mathrm{SFU}$, respectively. Distributions of the high- and low- frequency spectral indices for both groups are presented in Figure 16, bottom. For high frequency spectral index (negative region), the CSF median value is -2.1 , and $50 \%$ probability range lies between -4.1 and -1.1 . The median value for the reference group is $-2.3 ; 50 \%$ range for the reference flares is narrower and falls between -3.3 and -1.6 . Though the medians for the CSFs and reference group became closer to each other, the maximum between -2.0 and 0.0 for CSFs distribution still remains, thus the conclusion that there are significantly harder events among the CSFs is confirmed. The median value of the low-frequency spectral index (positive region) of CSFs is 2.0, which is larger than for the reference group characterized by the median value of 1.6 and coincides with the maximum of the CSFs distribution. Likewise the high-frequency spectral index distribution, the low-frequency one has a wider $50 \%$ range, 1.6-3.0, for the CSFs than for the reference set distribution, 0.9-2.6. We attempted a few other selections of the time frames for the statistical analysis, but did not find any trend differing from those reported above.

\subsection{Cold Solar Flare Timescales in Microwaves}

To characterize the timescales of the microwave bursts we used the same approach as Nita et al. (2004), who calculated the duration at the absolute peak frequency (see Nita et al. 2004, for more detail). For events with multiple temporal peaks the main peak was taken. This approach allows using the built-in OVSA_Explorer functionality for the peak duration determination and then to compare directly our results to those obtained by Nita et al. (2004). An unavoidable down side of this approach is that it can only be applied to 15 of 26 flares, for which the spectral fits were obtained. The results are listed in Table 6 (column $\Delta \mathrm{t}$ ) and presented in Figure 17. Microwave peak durations for CSFs extend from $7 \mathrm{~s}$ to $195 \mathrm{~s}$, but most events last less than $30 \mathrm{~s} .2000-$ Mar-18, 2000-May-18, 22:59 UT, and 2014-Oct-18 flares, the sameas in the HXR range, have a relatively longer duration. An estimated median value of the burst duration is $16 \mathrm{~s}$. This is much shorter compared to the reference flares, for which the median value 8 is $104 \mathrm{~s}$ and the durations extend up to thousands of seconds.

\subsection{Relationships between X-ray and Microwave Flare Parameters}

HXRs are often associated with the injected population of flare-accelerated nonthermal electrons, while the microwaves with the trapped component (Kosugi et al.

8 The value of $24 \mathrm{~s}$ in Table 2 in Nita et al. (2004) is incorrect likely due to a typo. 


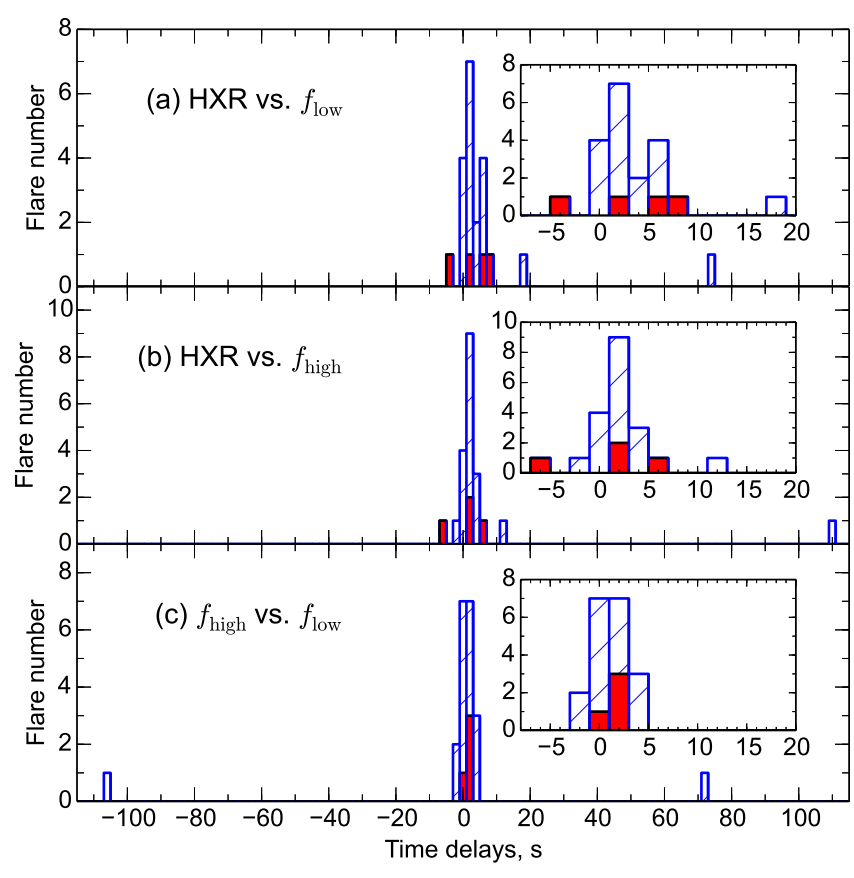

FIG. 18. - Time delays between HXR and microwave time profiles for cold solar flares. Red bins indicate the fraction of flares with only RSTN data in microwave range available. (a) Delays between time profile in G2 (HXR) and time profile at lowest microwave frequency, positive delay means that HXR emission is ahead. (b) Delays between time profile in G2 (HXR) and time profile at highest microwave frequency, positive delay means that HXR emission is ahead. (c) Delays between time profile at highest microwave frequency and lowest microwave frequency, positive delay means that microwave emission at highest frequency is ahead.

1988). Thus, the study of relative timing and relationships between HXR and microwave spectral characteristics can shed light on properties of both these important ingredients of solar flares and therefore conditions of nonthermal electron propagation in flaring loops.

\subsubsection{Time Delays between Microwave and HXR Emission}

Delays of microwave emission relative to HXR emission often indicate trapping of nonthermal electrons in flaring loops. To calculate these delays we used the Konus- Wind HXR time profiles in G2 channel because this channel is not contaminated by thermal emission. In the microwave range, we took time profiles at the highest frequency, $f_{\text {high }}$, where microwave burst was observed, which corresponds to optically thin gyrosynchotron emission. In addition to $f_{\text {high }}$, we examined microwave emission delays relative to HXR emission at the lowest frequency with observable gyrosynchotron emission, $f_{\text {low }}$. The frequencies $f_{\text {low }}$ and $f_{\text {high }}$ were selected for each flare via visual inspection.

The Konus-Wind time profiles were corrected for the light propagation time (see Section 3.1) and then interpolated to have the same time bins as the microwave time profiles. A lag-correlation between a HXR light curve and a microwave light curve was calculated using IDL function c_correlate, then the correlation coefficient dependence on the time delay was cubic-splineinterpolated with a step of $0.1 \mathrm{~s}$. The time lag corresponding to the peak of this function was adopted as the time delay between the HXR and microwave light curves. Time delays between the HXR and microwave light curves were obtained for 21 of 26 events for which appropriate microwave data were available. For the remaining 5 events it was not possible to compute the delays because of the low signal-to-noise ratio and faults in microwave data.

The results are presented in Table 6 and Figure 18 Histogram bin width was selected to be $2 \mathrm{~s}$, which is two times of NoRP background mode data resolution available for the majority of flares. For most CSFs microwave emission both on $f_{\text {low }}$ and on $f_{\text {high }}$ is delayed relative to HXR, for 4 flares microwave and HXR maxima coincide within $1 \mathrm{~s}$, and for 2 flares light curves on $f_{\text {high }}$ are ahead of HXR, but for one of these flares, only the RSTN data in microwave range are available, so the corresponding time delay may not be reliable. In most cases the delays of microwave emission do not exceed $10 \mathrm{~s}$, the most frequent delays are between 1 and $3 \mathrm{~s}$. One flare, 2000May-18, 22:59 UT, based on NoRP has larger delays: $18.4 \mathrm{~s}$ on $f_{\text {low }}$ and $12.8 \mathrm{~s}$ on $f_{\text {high }}$.

One flare, 2003-Oct-23, shows a smooth component that demonstrates a significant delay of the microwave $f_{\text {low }}$ emission relative to the HXR emission, $\sim 75 \mathrm{~s}$, Figure 19, left. This is the flare, whose smooth component (top left dynamic spectrum in Figure 14) was subtracted as a background in our analysis performed so far. Now we consider the microwave emission as it is, subtracting the preflare background only. The time profiles in the Konus- Wind G2 channel and at high microwave frequencies $(\geq 9 \mathrm{GHz})$ demonstrate impulsive behavior, while the microwave emission at the lower frequencies has a smooth time profile. This behavior is similar to that of the cold flare with a delayed heating described by Fleishman et al. (2016). Given the high flux density and steep slopes of the spectrum at both low and high frequencies the emission is clearly nonthermal and, thus, this flare likely contains a nonthermal electron population trapped in a relatively large magnetic flux tube.

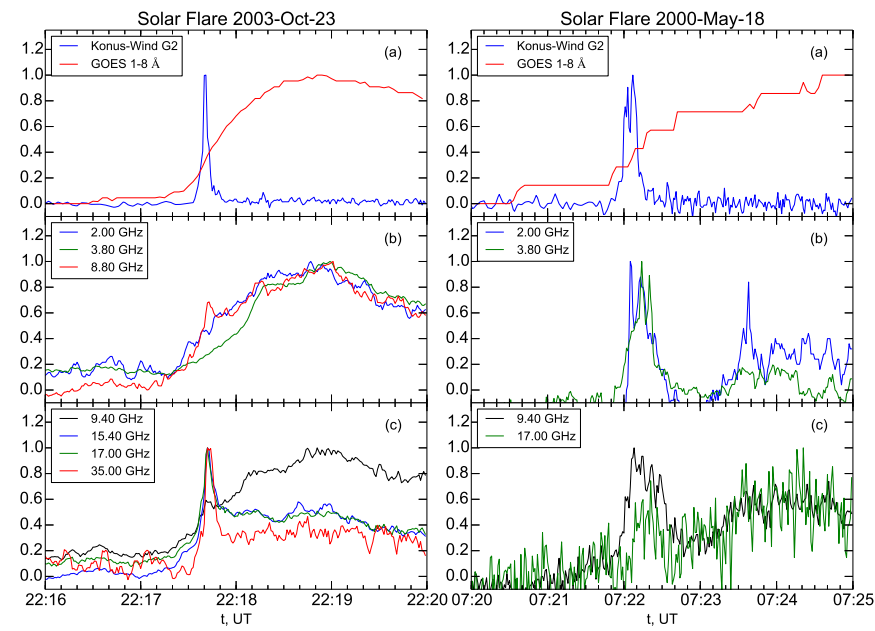

Fig. 19. - Example of solar flares with relatively large time delays between HXR and microwave emission. Left panel: solar flare 2003-Oct-23, $\mathrm{t}_{0}=22: 17: 42.391 \mathrm{~s}$, UT. Right panel: solar flare 2000-May-18, $\mathrm{t}_{0}=07: 21: 57.787 \mathrm{~s}, \mathrm{UT}$. (a) Konus-Wind G2 channel and GOES 1-8 $\AA$ light curves. (b) Microwave light curves on lower $(\leq 9 \mathrm{GHz})$ frequencies. (c) Microwave light curves on higher $(\geq 9 \mathrm{GHz})$ frequencies. 
On the contrary, the 2000-May-18 07:21 UT, flare has a relatively large delay between the HXR and microwave time profiles at the high frequency, Figure 19, right. For this event the light curves in the HXR G2 channel and microwave at lower frequencies (2.0 and $3.8 \mathrm{GHz})$ are highly correlated, but the emission on $f_{\text {high }}$ is more gradual and its maximum is delayed relative to the impulsive phase by $\sim 110 \mathrm{~s}$. This microwave burst is rather weak, $\sim 10 \mathrm{sfu}$, and, thus, the delayed component could be produced by the flare-heated plasma by either free-free or gyro- emission mechanism; cf. thermal flare reported by Fleishman et al. (2015).

The microwave time profiles at $f_{\text {low }}$ and $f_{\text {high }}$ do not show any delay between each other for 7 events, while for most of the flares (10 cases) the light curves at the lower microwave frequencies are delayed relative to the higherfrequency ones. There are a few possible effects that can be responsible for such a delay. For example, it can be due to rise of the brightness temperature of the optically thick low-frequency emission due to nonthermal electron spectral hardening (Melnikov 1994) or due to decrease of the free-free opacity provided by plasma heating in the case of cold dense flares (Bastian et al. 2007).

\subsubsection{Relationship between the flare duration in Microwave and $H X R$}

Relationship between flare timescales in HXR and microwave ranges is presented in Figure 20. The events are split onto two groups: for one of them points are close to the solid line, which represents the equality of HXR and microwave durations, while the other group demonstrates the microwave burst significantly longer than the corresponding HXR burst. A simple interpretation of these trends is that the first group is composed of trappingfree events, while the trapping plays some role in the events from the second group.

\subsubsection{Microwave vs. HXR spectral indices}

Here we compare the high-frequency microwave spectral indices $\alpha_{\mathrm{hf}}$, which correspond to the optically thin gyrosynchotron emission and, thus, closely linked with the spectral indices of nonthermal electron distribution in the flaring loop, and the HXR spectral indices $\gamma$, which are associated with the spectral indices of injected electrons.

Microwave spectral parameters were obtained on $1 \mathrm{~s}$ time scales, but the multichannel HXR spectra with such time resolution were not available for the majority of flares, thus, the power-law indices in the HXR range, $\gamma$, were calculated using 3-channel fitting (see Section 3.1) on the time intervals in Konus- Wind G1, G2, G3 channels corresponding to the intervals with successful microwave fits after correction for the propagation time. HXR fit results with fit probabilities $<1 \%$ were discarded. Surprisingly, the scatter plot (not shown) of $\alpha_{\text {hf }}$ vs $\gamma$ does not reveal any significant correlation between these two parameters; so we investigate this relationship on the event-by-event basis.

It is known (Trottet \& Vilmer 1984; Melnikov \& Magun 1998) that for some flares the HXR and microwave spectral indices behave consistently at the rise phase but show opposite trends at the decay phase, which has been interpreted as an outcome of Coulomb collisions of the trapped population of the

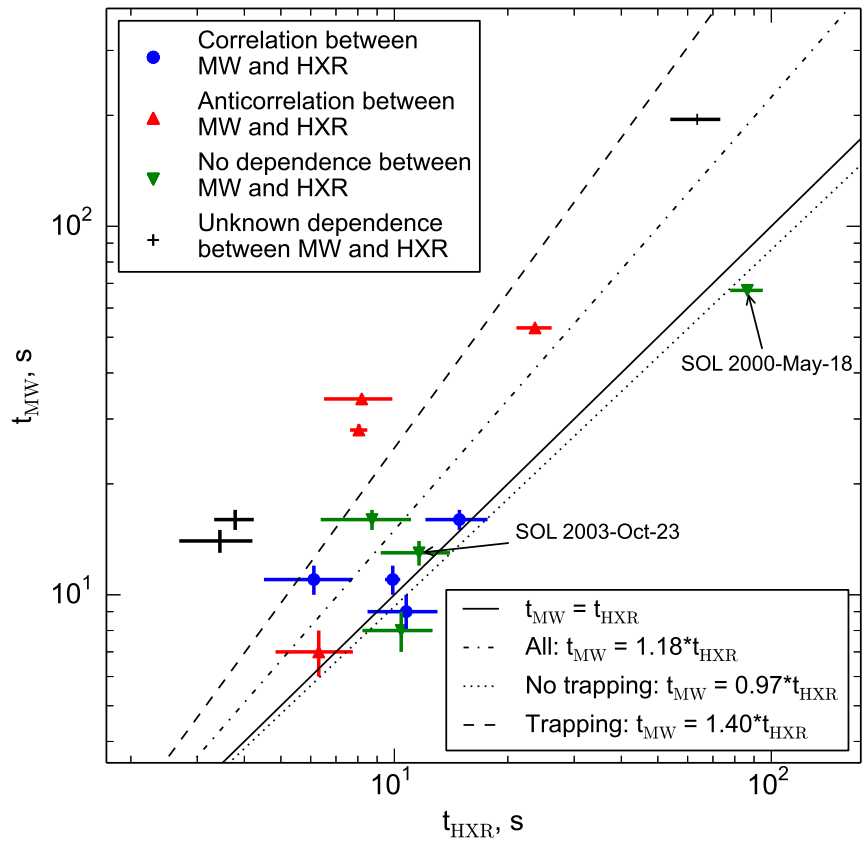

FIG. 20.- Relationship between CSFs timescales in HXR and microwave. Solid line represents duration equality, dash-dotted line corresponds to linear regression between these timescales, dotted line reflects regression for the group of flares below the main regression (presumably without trapping), dashed line indicates regression for the flares above the main regression line (presumably with trapping). Explanation of "colored" event groups is given in Section 3.6 .3

nonthermal electrons with the ambient plasma particles. For this reason we consider the relationships between microwave high frequency spectral index, $\alpha_{\mathrm{hf}}$, and HXR index, $\gamma$, separately for the rise and decay phases of microwave emission.

The data suitable for comparison between $\alpha_{\mathrm{hf}}$ and $\gamma$ were obtained for twelve out of fifteen CSFs with successful microwave fits. Three flares were excluded because no triggered mode data were available in the G2 and G3 channels for the 2014-Oct-18 flare, while the high frequency spectral indices could not be obtained reliably for the 1998-May-07 and 1999-Dec-02 flares due to small number of the spectral data points or due to a weak signal to the right of the turnover frequency. Time frames with weak signal or only one data point above the spectral peak frequency were excluded (recall, these same time frames contribute to the "tails" of $\alpha_{\mathrm{hf}}$ distributions as has been discussed in Section 3.4).

The results of this analysis are presented in Figure 21. All CSFs in general follow the soft-hard-soft spectral evolution in HXR range, while according to their spectral evolution in microwave range and the relationship between $\gamma$ and $\alpha_{\mathrm{hf}}$, CSFs can be roughly categorized onto three groups. The first group (the first column in Figure 21] includes flares 2001-Nov-01, 2011-Sep-19, 2013Nov-05, and 2014-Jan-02, for which the correlation between $\alpha_{\mathrm{hf}}$ and $\gamma$ is observed. Of these four flares, the 2001-Nov-01 flare and 2013-Nov-05 flare show a slight correlation between the spectral indices in the microwave and HXR ranges during both the rise and the decay phases, while for other two flares from this group no relationship during the rise phase is revealed. The sec- 

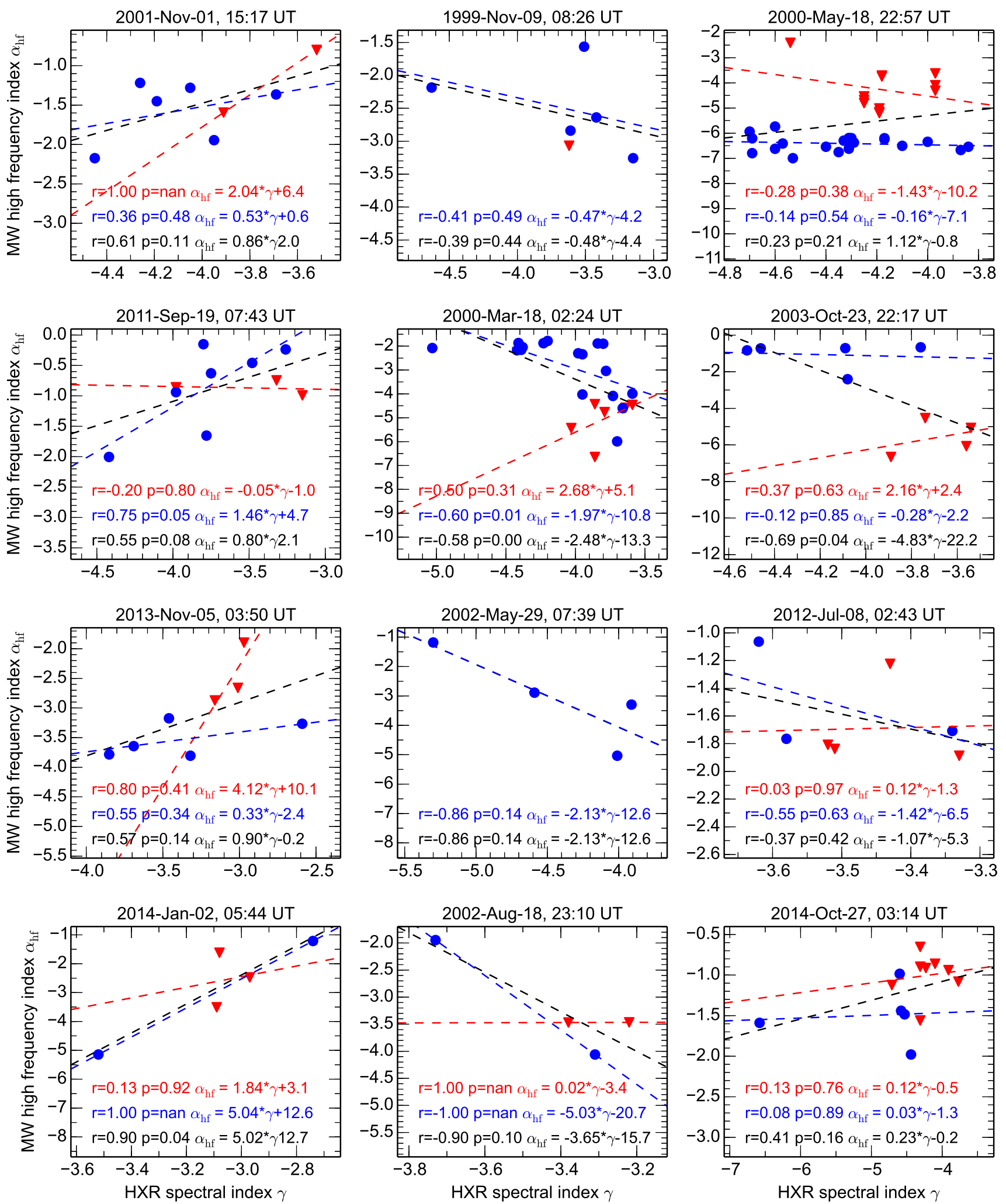

FIG. 21.- Relationship between HXR spectral index $\gamma$ and microwave high frequency index $\alpha_{h f}$ obtained on each time interval during peak with successful microwave and HXR spectral fitting. HXR spectral indices with high temporal resolution were obtained using 3channel fitting of Konus-Wind data (see Sec. 3.1). Red triangles and blue circles correspond to rise and decay phases of microwave emission correspondingly. Red labels indicate relationships between $\gamma$ and $\alpha_{h f}$ on the rise phase, blue labels indicate relationships on the decay phase, black labels indicate relationships during the whole flare. 
ond group (the second column in Figure 21) includes 1999-Nov-09, 2000-Mar-18, 2002-May-29, 2002-Aug-18 flares, which are characterized by an anticorrelation between $\alpha_{\mathrm{hf}}$ and $\gamma$ during the decay phase. One flare of this group, 2000-Mar-18, shows correlation between microwave and HXR indices during the rise phase, while for other three flares the corresponding regressions at the rise phase either do not show any clear trend or do not contain enough data points to draw a conclusion. The third group (the third column in Figure 21) includes 2000-May-18, 22:57 UT, 2003-Oct-23, 2012-Jul-08, and 2014-Oct-27 flares, which do not show any clear dependence between the microwave and HXR spectral indices during both the rise and the decay phases. An interesting feature of 2000-May-18, 22:57 UT and 2003-Oct23 flares of this group is a striking difference between $\alpha_{\mathrm{hf}}$ during the rise and decay microwave phases. For the 2000-May-18, 22:57 UT flare the spectral indices are harder at the rise phase as compared to the decay phase, while for the 2003-Oct-23 flare, the microwave spectral indices are harder during the decay phase. Thus, we can conclude that for the flares from the second group and for one flare from the third group, the microwave spectrum hardening is observed. On the contrary, for flares from the first group and for one flare from the third group the microwave spectrum becomes softer during the flare.

There is a correspondence between the flare duration patterns revealed by Figure 20 and the spectral evolution patterns identified in Figure 21, in terms of presence or absence of the trapping in the given event. Indeed, the events elongated along the $y=x$ duration equality line in Figure 20 are mainly from columns 1 (three events) and 3 (three events) in Figure 21 with only one event from column 2. Three other events from column 2 (showing a microwave spectrum hardening at the decay phase indicative of spectral evolution of the trapped component of nonthermal electrons) are those with noticeably longer duration in microwave than in the HXR domain, which confirms that trapping plays a role in these flares.

\subsubsection{Relationships between X-ray characteristics and Microwave peak frequencies}

In Section 3.4 we found that the CSFs have strikingly higher spectral peak frequencies $f_{\text {peak }}$ compared to the reference set of the microwave bursts. From this perspective it looks interesting to consider if any other CSF parameter correlates with the microwave spectral peak frequency.

To compare the microwave spectral peak frequencies obtained on individual time frames with X-ray parameters characterising the entire duration of the solar flare, the peak frequencies for flares with successful fits were averaged over time frames for each flare peak; the corresponding uncertainty of the spectral peak frequency was estimated as the standard deviation from this mean. For the 'no-fit' flares, in some cases it was possible to specify a lower limit of the spectral peak frequency directly from the dynamic spectra given in Figure 14.

The relationship between the thermal-nonthermal (TNT) ratio $\eta=\Delta \mathrm{GOES} /(\mathrm{HXR}$ peak count rate) and the mean microwave peak frequencies is displayed in Figure 22. This plot shows, that most of the CSFs group between $\sim 5 \mathrm{GHz}$ and $\sim 20 \mathrm{GHz}$ in the peak frequency and between $\sim 4 \times 10^{-11}$ and $\sim 1 \times 10^{-10}$ in the TNT ratio (see

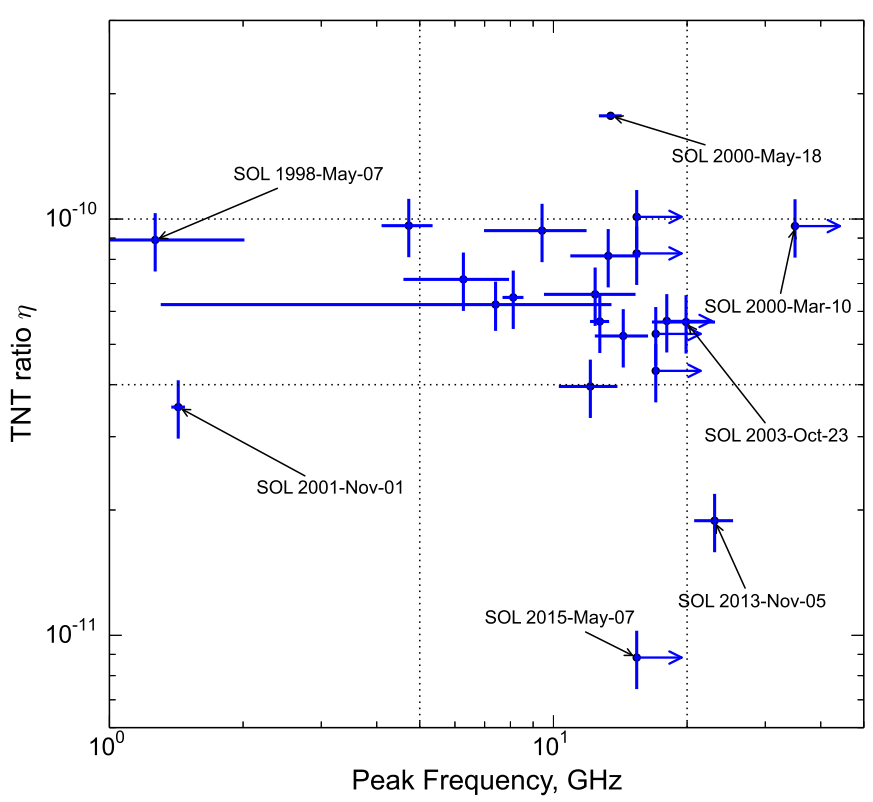

FIG. 22.- Relationship between $\triangle$ GOES to Konus-Wind HXR peak count rate ratio and microwave peak frequency. Microwave peak frequencies were averaged over time frames with successful microwave fits for each flare and error bars refer to their standard deviations. For flares with fail fits where it was possible lower limits of peak frequency were estimated.

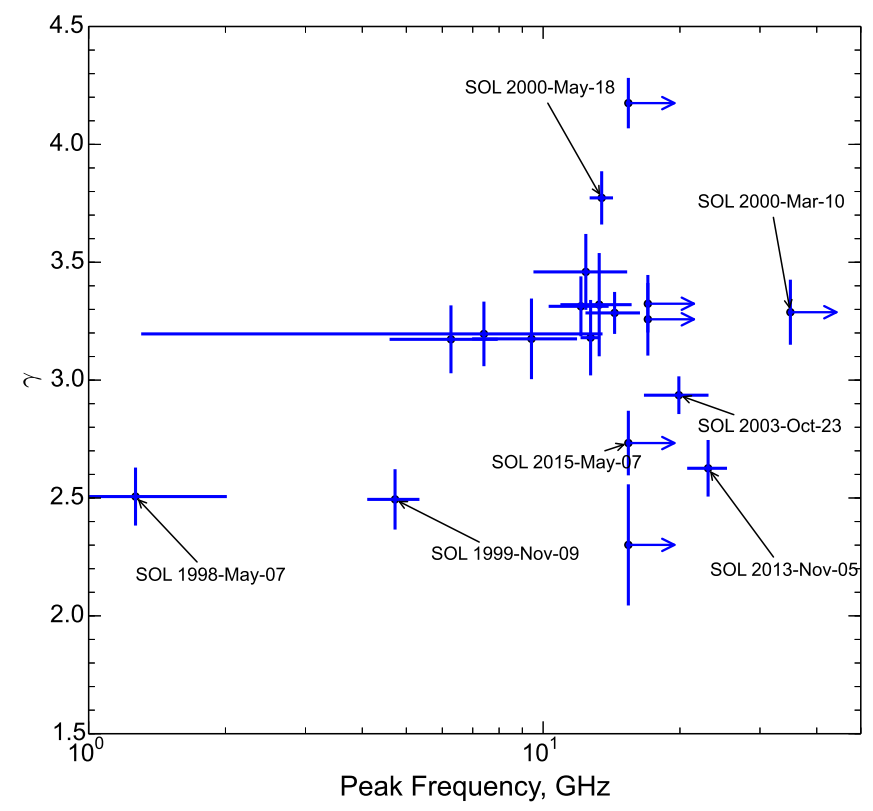

FIG. 23.- Relationship between photon power index in lower energy range $\gamma$ and microwave peak frequency. Microwave peak frequencies were averaged over time frames with successful microwave fits for each flare and error bars refer to their standard deviations. For flares with fail fits where it was possible lower limits of peak frequency were estimated.

dotted lines in the plot). Some flares fall outside these ranges. These are the 2000-May-18 flare, which has a rather high TNT ratio, the 2001-Nov-01 flare with a low $\triangle$ GOES to HXR peak count rate TNT ratio and a low mean peak frequency $(1.4 \mathrm{GHz})$; the 2013-Nov-05 flare that demonstrates a low TNT ratio and an exceptionally 


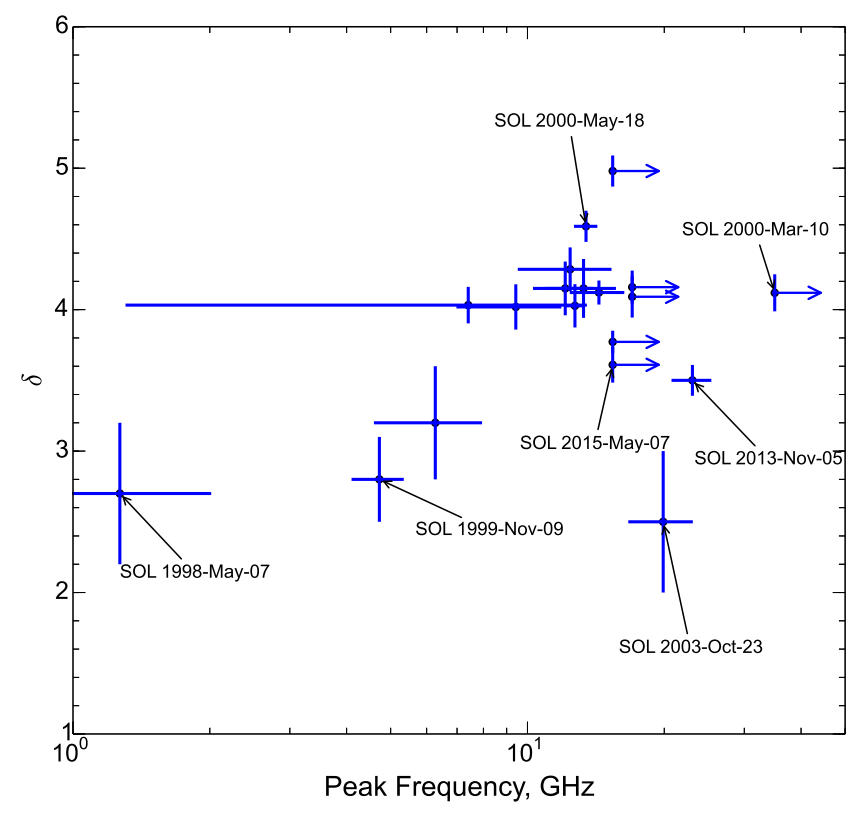

FIG. 24. - Relationship between electron power index in lower energy range $\delta$ and microwave peak frequency. Microwave peak frequencies were averaged over time frames with successful microwave fits for each flare and error bars refer to their standard deviations. For flares with fail fits where it was possible lower limits of peak frequency were estimated.

high mean peak frequency, $22 \mathrm{GHz}$; and the 2015-May-07 flare with an exceptionally low TNT ratio.

The relationship between the photon power index in the lower energy range, $\gamma$ for $\mathrm{PL}$ model or $\gamma_{1}$ for $2 \mathrm{PL}$ model and the mean microwave peak frequencies, is presented in Figure 23. The lower-frequency CSFs tend to be harder than the higher-frequency ones. There is no case, where a soft spectrum and a low spectral peak frequency would be present simultaneously.

The relationship between the electron power index in the lower energy range, $\delta$ for brmThickPL model or $\delta_{1}$ for brmThick2PL model and the mean microwave peak frequencies, is presented in Figure 24. There is a slight tendency that events with lower peak frequencies has harder $\delta$. Here we see the same trend as in the previous scatter plot of $\gamma$ vs $f_{\text {peak }}$.

\section{DISCUSSION}

In this study we have identified a statistically significant set of "cold" flares with a disproportionally weak thermal relative to the nonthermal emission. Although a few such cold flares were reported in a number of case studies, all those cases were selected subjectively without any formal criterion. Thus, to perform this statistical study, we have started with formulating such a formal criterion, which appears to be rather strict. Specifically, we identified a group of early impulsive flares by selecting those events, in which the HXR burst started before or without the corresponding SXR GOES flare. Then, we computed a SXR enhancement during the HXR bursts and compared this enhancement for a referent set of bursts and early impulsive flares. Finally, we selected the early impulsive flares, which are also the outliers (far below 'average' thermal response) in the scatter plots in Figure 5, to form our CSF list. Note that Figure 5] con- tains many more outliers below the confidence interval, which are not early impulsive flares according our selection criterion. We, however, did not add them to the list because for the non-early impulsive flares, such a relatively weak GOES enhancement during the HXR burst could be an outcome of a strong pre-heating (i.e., the overall GOES enhancement is strong, but the enhancement due to nonthermal electrons is weak) - such flares, certainly, would not qualify as the cold ones. This selection yielded 27 CSFs, which we analyzed using X-Ray and microwave data and the cross-correlations between them.

The performed statistical study reveals significant differences between the CSFs and other flares. In the HXR domain the CSFs are harder, shorter, and weaker than the reference flares. In the microwave domain the CSFs are, however, not weaker than the referent bursts. Further, in the microwave domain the CSFs are shorter and harder at high frequencies than the reference ones, while steeper at the low frequencies. In addition, the CSFs often have a strikingly higher spectral peak frequency than the reference ones in the microwave domain. Nevertheless, we found that CSFs do not represent a uniform group of evens, but rather could be separated onto a few subclasses. In particular, some CSFs show signatures of the nonthermal electron trapping in a coronal flaring loop, while others do not show any trapping (as in the main flaring loop in the flare reported by Fleishman et al. 2016); some flares demonstrate a break in the nonthermal electron energy spectrum, while others are consistent with a single power-law; some CSFs are likely produced in a dense source as in the cases reported by Bastian et al. (2007); Masuda et al. (2013), while others - in a tenuous one similar to the tenuous flare reported by Fleishman et al. (2011).

Perhaps, the key to interpret these distinctions is the combination of a weaker intensity in HXR and normal intensity in microwave. Indeed, weaker HXR emission implies a weaker component of the nonthermal electrons accelerated in the flare. But, to produce a normal level of microwave gyrosynchotron emission by a weaker population of nonthermal electrons, the magnetic field must be accordingly higher than in the referent flare. The stronger magnetic field further implies a higher spectral peak frequency of gyrosynchotron emission as observed. Then, given that the magnetic field decreases with height in the corona, a strong magnetic field implies a reasonably low height of the radio source, thus, shorter flaring loops, and thus, shorter burst duration. These more compact loops represent more uniform sources, i.e., they likely contain a narrower range of magnetic field strength than a bigger loop, which explains, why the lowfrequency microwave slope is steeper in the CSFs compared with the referent ones. This simple interpretation cannot, however, clarify, what is the reason of harder spectra of nonthermal electrons in the CSFs compared with the referent ones. One possibility is that the acceleration mechanism results in harder spectra in case of stronger magnetic field / more compact loops. An alternative is the spectral hardness does not systematically depend on these flare parameters, but in the flares that generate electrons with harder spectra, the thermal response is additionally reduced because now a bigger fraction of the nonthermal energy belongs to high-energy 
electrons, which deposit their energy deeper in the chromosphere, thus reducing the chromospheric evaporation and the thermal response.

However, even the relatively compact set of $27 \mathrm{CSFs}$ demonstrates a considerable diversity of the properties, so that the CSFs can hardly be fully characterized by a "mean" CSF, but rather have to be further split onto different subclasses. This is particularly true for those CSFs showing a normal or low microwave spectral peak frequency: in such cases the magnetic field is also supposed to be normal or weak, so the picture drawn above should be patched or replaced, although the short duration still implies a reasonable compact flaring sources. We note, that in such cases, the nonthermal electron spectra are particularly hard, which might further confirm the role of the spectral hardness in the chromospheric evaporation / thermal response. We note that having a low spectral peak frequency requires that both magnetic field and the plasma density are low. Indeed, a weak magnetic field combined with a high plasma density will result in a high spectral peak frequency due to the Razin-effect. Thus, the flares with a low spectral peak frequency are likely tenuous flares similar to that reported by Fleishman et al. (2011).

Another property, which display different patterns within CSFs is the shape of the nonthermal electron energy spectrum. At this point we cannot draw any conclusion about significance of this finding given that in some cases the spectral break can be present in the spectrum, but not recovered in the fit due to insufficient statistics at the high-energy channels.

Finally, we found that the CSFs can be further divided onto two groups, depending on whether the nonthermal electron trapping in a coronal flaring loop (or loops) plays a role or not. This is vividly demonstrated by Figure 20 showing the scatter plot of the microwave vs HXR burst duration. Roughly half of the events show equality of the durations (no trapping), while in the other half the microwave duration is a factor of two longer than the HXR duration (a noticeable trapping). This division is also confirmed by relationships of the spectral hardness in the microwave and HXR domains, Section 3.6.3.

\section{CONCLUSIONS}

From the performed statistical analysis we conclude that the our identified set of 27 "cold" solar flares demonstrates properties statistically different from those of a referent ("mean") flare. We found that the cold flares are typically shorter and harder that the referent flares; their HXR emission is weaker, while microwave emission is comparable to that of the mean flare. They are further different in the microwave domain as they have a steeper low-frequency spectral slope and (often) strikingly higher spectral peak frequency. From these findings we conclude that the cold flares are typically produced in more compact structures (presumably, short flaring loops) having stronger magnetic fields, than a mean flare. In addition, we found that the group of the cold flares is nonuniform, but rather can be further sub-categorized according to various properties (low or high spectral peak frequency, presence or absence of trapping effect etc). Given that all these flares demonstrate the weakest thermal response compared with a referent flare, we conclude that the described here "cold" flares offer, via the corresponding case studies, the cleanest way to study the electron acceleration in flares and the thermal plasma response driven by the nonthermal electron population.

We note that the presented here CSFs form a promising event list for future case studies given that the processes of particle acceleration and the thermal plasma response can be quantified much cleaner with the nonthermal-energy-dominated CSFs than with a 'usual' flare, where the nonthermal and thermal energies are initially mixed up with unknown proportions. Such case studies will employ all available imaging and contextual data and also incorporate modeling to verify and refine interpretations formulated here based on the statistical analysis.

We are highly grateful to Dr. Valentin Pal'shin for initiation of this work and his help with Konus- Wind data. We thank anonymous referees who helped us to improve the paper. We thank Dr. Dmitry Svinkin, Dr. Dmitry Frederiks and Mr. Mikhail Ulanov for useful advice concerning analysis of the Konus-Wind data, Prof. Astrid Veronig for productive discussion and Dr. Gelu Nita for providing microwave data from their paper and consultations. AL gratefully acknowledge support from RSF grant 17-12-01378. GF thanks NSF grants AGS-1250374 and AGS-1262772, NASA grant NNX14AC87G to New Jersey Institute of Technology and RFBR grant 15-0203835. NM acknowledge RFBR grants 15-02-03717 and 15-02-08028. AT and DZ acknowledge support from the Ministry of Education and Science of Russian Federation and from Siberian Branch of the Russian Academy of Sciences (Project II.16.3.2) and from the Program of basic research of the RAS Presidium No.28. Experimental data were obtained using the Common Use Centre "Angara" equipment.

\section{REFERENCES}

Akritas, M. G. \& Bershady, M. A. 1996, ApJ, 470, 706

Aptekar, R. L., Frederiks, D. D., Golenetskii, S. V., Ilynskii, V. N., Mazets, E. P., Panov, V. N., Sokolova, Z. J., Terekhov, M. M., Sheshin, L. O., Cline, T. L., \& Stilwell, D. E. 1995, Space Sci. Rev., 71, 265

Arnaud, K. A. 1996, in Astronomical Society of the Pacific Conference Series, Vol. 101, Astronomical Data Analysis Software and Systems V, ed. G. H. Jacoby \& J. Barnes, 17 Aschwanden, M. J. 2011, Sol. Phys., 274, 99

Bai, T. \& Sturrock, P. A. 1989, ARA\&A, 27, 421

Bastian, T. S., Fleishman, G. D., \& Gary, D. E. 2007, ApJ, 666, 1256

Battaglia, M., Fletcher, L., \& Benz, A. O. 2009, A\&A, 498, 891

Bevington, P. R. 1969, Data reduction and error analysis for the physical sciences, 208-209
Brown, J. C. 1971, Sol. Phys., 18, 489

Crosby, N. B., Aschwanden, M. J., \& Dennis, B. R. 1993, Sol. Phys., 143, 275

Dennis, B. R. 1985, Sol. Phys., 100, 465

Domingo, V., Fleck, B., \& Poland, A. I. 1995, Sol. Phys., 162, 1

Fleishman, G. D., Kontar, E. P., Nita, G. M., \& Gary, D. E. 2011, ApJ, 731, L19

Fleishman, G. D., Nita, G. M., \& Gary, D. E. 2015, ApJ, 802, 122

Fleishman, G. D., Pal'shin, V. D., Meshalkina, N., Lysenko,

A. L., Kashapova, L. K., \& Altyntsev, A. T. 2016, ApJ, 822, 71 Garcia, H. A. 1994, Sol. Phys., 154, 275

Gary, D. E. \& Hurford, G. J. 1989, ApJ, 339, 1115

—. 1994, ApJ, 420, 903 
Grechnev, V. V., Lesovoi, S. V., Smolkov, G. Y., Krissinel, B. B., Zandanov, V. G., Altyntsev, A. T., Kardapolova, N. N.,

Sergeev, R. Y., Uralov, A. M., Maksimov, V. P., \& Lubyshev, B. I. 2003, Sol. Phys., 216, 239

Guidice, D. A., Cliver, E. W., Barron, W. R., \& Kahler, S. 1981, in Bulletin of the American Astronomical Society, Vol. 13, Bulletin of the American Astronomical Society, 553

Holman, G. D., Aschwanden, M. J., Aurass, H., Battaglia, M., Grigis, P. C., Kontar, E. P., Liu, W., Saint-Hilaire, P., \& Zharkova, V. V. 2011, Space Sci. Rev., 159, 107

Hurford, G. J., Read, R. B., \& Zirin, H. 1984, Sol. Phys., 94, 413

Kontar, E. P., MacKinnon, A. L., Schwartz, R. A., \& Brown, J. C. 2006, A\&A, 446, 1157

Kosugi, T., Dennis, B. R., \& Kai, K. 1988, ApJ, 324, 1118

Kouveliotou, C., Meegan, C. A., Fishman, G. J., Bhat, N. P., Briggs, M. S., Koshut, T. M., Paciesas, W. S., \& Pendleton, G. N. 1993, ApJ, 413, L101

Lin, R. P., Dennis, B. R., Hurford, G. J., Smith, D. M., Zehnder, A., Harvey, P. R., Curtis, D. W., Pankow, D., Turin, P., Bester, M., Csillaghy, A., Lewis, M., Madden, N., van Beek, H. F., Appleby, M., Raudorf, T., McTiernan, J., Ramaty, R., Schmahl, E., Schwartz, R., Krucker, S., Ảbiad, R., Quinn, T., Berg, P., Hashii, M., Sterling, R., Jackson, R., Pratt, R., Campbell, R. D., Malone, D., Landis, D., Barrington-Leigh, C. P., Slassi-Sennou, S., Cork, C., Clark, D., Amato, D., Orwig, L., Boyle, R., Banks, I. S., Shirey, K., Tolbert, A. K., Zarro, D., Snow, F., Thomsen, K., Henneck, R., McHedlishvili, A., Ming, P., Fivian, M., Jordan, J., Wanner, R., Crubb, J., Preble, J., Matranga, M., Benz, A., Hudson, H., Canfield, R. C., Holman, G. D., Crannell, C., Kosugi, T., Emslie, A. G., Vilmer, N., Brown, J. C., Johns-Krull, C., Aschwanden, M., Metcalf, T., \& Conway, A. 2002, Sol. Phys., 210, 3

Masuda, S., Shimojo, M., Kawate, T., Ishikawa, S.-n., \& Ohno, M. 2013, PASJ, 65, 1

Melnikov, V. F. 1994, Radiophysics and Quantum Electronics, 37, 557

Melnikov, V. F., Gary, D. E., \& Nita, G. M. 2008, Sol. Phys., 253, 43

Melnikov, V. F. \& Magun, A. 1998, Sol. Phys., 178, 153

Motorina, G. G., Kontar, E. P., \& Fleishman, G. D. in preparation, ApJ
Muratov, A. A. 2011, in Baikal Young Scientists' International School, Vol. 365, XIIth Conference of Young Scientists, 85-87

Nakajima, H., Nishio, M., Enome, S., Shibasaki, K., Takano, T. Hanaoka, Y., Torii, C., Sekiguchi, H., Bushimata, T.,

Kawashima, S., Shinohara, N., Irimajiri, Y., Koshiishi, H.,

Kosugi, T., Shiomi, Y., Sawa, M., \& Kai, K. 1994, IEEE

Proceedings, 82, 705

Nemmen, R. S., Georganopoulos, M., Guiriec, S., Meyer, E. T., Gehrels, N., \& Sambruna, R. M. 2012, Science, 338, 1445

Neupert, W. M. 1968, ApJ, 153, L59

Nita, G. M., Gary, D. E., \& Lee, J. 2004, ApJ, 605, 528

Pal'shin, V. D., Charikov, Y. E., Aptekar, R. L., Golenetskii, S. V., Kokomov, A. A., Svinkin, D. S., Sokolova, Z. Y., Ulanov, M. V., Frederiks, D. D., \& Tsvetkova, A. E. 2014,

Geomagnetism and Aeronomy, 54, 943

Shramko, A. D., Senik, V. A., \& Tlatov, A. G. 2011, Cosmic Research, 49, 93

Silva, A. V. R., Wang, H., \& Gary, D. E. 2000, ApJ, 545, 1116

Somov, B. V. \& Syrovatskii, S. I. 1976, Soviet Physics Uspekhi, 19,813

Stahli, M., Gary, D. E., \& Hurford, G. J. 1989, Sol. Phys., 120, 351

Su, Y., Gan, W. Q., \& Li, Y. P. 2008, Advances in Space Research, 41, 988

Sui, L., Holman, G. D., \& Dennis, B. R. 2007, ApJ, 670, 862

Tanaka, K., Watanabe, T., Nitta, N., \& Akita, K. 1983,

Sol. Phys., 86, 91

Torii, C., Tsukiji, Y., S., K., Yoshimi, N., Tanaka, H., \& Enome, S. 1979, Proc. of the Res. Ist. of Atmospherics, Nagoya Univ., 26,129

Trottet, G. \& Vilmer, N. 1984, Advances in Space Research, 4, 153

Veronig, A. M., Brown, J. C., Dennis, B. R., Schwartz, R. A., Sui, L., \& Tolbert, A. K. 2005, ApJ, 621, 482

White, S. M., Kundu, M. R., Bastian, T. S., Gary, D. E., Hurford, G. J., Kucera, T., \& Bieging, J. H. 1992, ApJ, 384, 656

White, S. M., Thomas, R. J., \& Schwartz, R. A. 2005, Sol. Phys., 227,231

Zhdanov, D. A. \& Zandanov, V. G. 2011, Central European

Astrophysical Bulletin, 35, 223

-. 2015, Sol. Phys., 290, 287

APPENDIX

\section{CREATION OF THE OVSA-LIKE DATABASE OF THE COLD SOLAR FLARES}

A generic OVSA data file contains low- and high- time resolution data with the resolutions differing by a factor of two from all available antennas. There are OVSA tools developed specifically for reduction and analysis of the OVSA data. For our data set, the input microwave data are, however, nonuniform as they are taken by substantially different radio instruments. To partly reduce the effect of this data nonuniformity on the statistical results we made a number of manipulations with the input data. For the events jointly observed by more than one instrument we combined the data from the different instruments into one composite dynamic spectrum to increase spectral resolution needed for meaningful time-sequential spectral fit of the data. Given the dissimilar time resolution and distinct clock ticks at various instruments, we interpolated the time array of one instrument to exactly match the clock ticks of another instrument selected to be a reference one. Whenever possible we adopted the NoRP clock ticks to be the reference ones, and resampled the RSTN and/or SRS and BBMS data to exactly match the NoRP time.

We noted that for the full NoRP time resolution in the flare mode, $0.1 \mathrm{~s}$, the microwave light curves for many events are noisy at many frequencies. For this reason, we integrated the $0.1 \mathrm{~s}$ data to degrade the time resolution up to $0.5 \mathrm{~s}$, which is exactly half of the NoRP resolution in the background mode. Having the two sets of observations, one with $0.5 \mathrm{~s}$ resolution created from the flare mode data, and the other with $1 \mathrm{~s}$ resolution taken from the background mode data allows us to create a data file having the structure internally identical to the generic OVSA data file. For the events for which NoRP data are unavailable (or only available in the background mode), we created a dynamic spectrum with a single time resolution, $1 \mathrm{~s}$, which is also allowable by the OVSA data format.

Although a combination of more than two instruments is, in principle, possible for a given event, in practice we mainly created the composite data files containing the data from two different instruments; namely, combination of the NoRP and the RSTN data (7 events), the NoRP and the SRS data (2 events) or the NoRP and the BBMS data (1 event) and in one case the RSTN and the KMAS data, while the combination of the data from three different instruments (NoRP, RSTN, and SRS) was only available in two cases. Note, that there are often RSTN clock error: 9 , which can be as big as many seconds. We corrected these errors by cross-correlation between the RSTN and NoRP data, relying on the NoRP clocks as the most precise. For two events we built separate dynamic spectra from the OVSA data or the NoRP+RSTN combination and for one more from the OVSA and the NoRP separately. We did not add other instruments to the events observed with the OVSA, since it typically has sufficient number of the

9 This, in particular, implies that using RSTN data alone to measure the time delays between the X-ray and microwave light 
spectral channels to resolve and fit the burst spectrum without adding extra channels. However, considering a separate NoRP or NoRP + RSTN spectrum has an advantage of higher time resolution, which is important to analyze short and rapidly variable events. 